\title{
Distribution of Mantis religiosa (L.) and its changes in Poland ${ }^{*}$
}

\author{
Anna LIANA \\ Museum and Institute of Zoology,PAS, Wilcza 64,00-679Warszawa; e-mail: aliana@miiz.waw.pl
}

\begin{abstract}
Mantis religiosa has been a constant element of Polish fauna for a long time but its current expansion in Poland has only been observed for a few years. At first, population growth was recorded within an insular area in the central part of the Sandomierz Basin but the total Polish range of the species seemed to be unchanged. Next, the praying mantis was recorded in some adjacent regions where it was observed only sporadically in the 1950s (the Małopolska Upland, the Lublin Upland) and in a few distant ones where it had never been observed before (Beskid Niski Mts, Bieszczady Mts, Pogórze Karpackie [Carpathian Foothills], Polesie Lubelskie). A number of factors affect the spread of $M$. religiosa dispersion; the most important ones including climate warming during the past few decades, increase in area of fallow lands, and transport of various developmental stages, especially oothecae, by man.
\end{abstract}

Key words: Mantis religiosa, Poland, insular area, expansion, climate change, fallow lands

\section{INTRODUCTION}

Mantis religiosa (L.) was first recorded from Poland already in the 18th century, while the species has probably occurred in our country for centuries. However, numerous records concerning the occurrence of the praying mantis appeared only in the 1950s (Table 1), and made it possible to characterize its distribution in Poland (Bazyluk 1958, 1960). Due to the ephemeral nature of many localities and small size of the population and high rank of the taxon (as the only representative of the order Mantodea), appeals to make this species legally protected were already heard at that time (Michalski 1959).

The praying mantis was included in the Polish checklist of protected animals in 1984. At present, it is also included in "Red List of Threatened Animals in Poland" (Liana 1992, 2002) and in the two editions of "Polish Red Data Book of Animals" (Witkowski 1992, Liana 2004). On the basis of the data collected until the turn of the 21 st century, the species was considered to be CR (critically threatened: Liana 2002). More recent information proved it to belong to the EN category (endangered: Liana 2004). This fact reflects a rate of dispersion dynamics of the praying mantis recently observed in Poland. Such an expansion has been reported from western European countries even earlier, in the 1990s (Berg \& Keller 2004, Brechtel, Ehrmann \& Detzel 1996, Ehrmann 1984 and others).

In Poland, M. religiosa has always aroused interest among scientists. Its presence in the insular area in Kotlina Sandomierska has been investigated periodically. Attempts were made to find it also in the adjacent regions, e.g. in xerothermic habitats of Wyżyna Małopolska and Wyżyna Lubelska (Liana 1976, 1978), Góry Świętokrzyskie (the Holy Cross Mountains), Roztocze and Lasy Janowskie (the Janowskie Forest) (Liana 1992, 1994, 1997, 2002). In the light of these studies and the research conducted earlier in Mazovia (Liana 1966), Bieszczady

\footnotetext{
*The paper has been prepared as a result of research project sponsored by the Ministry of Science and Higher Education - grant No 2 P04G02226 (2004-2007). A preliminary inventory of praying mantis in Puszcza Sandomierska was a part of regional faunistic research commissioned by Province Governor's Office in Tarnobrzeg (1997-1999) and then by Province Governor's Office in Rzeszów (2003).
} 
and Pieniny (the Pieniny Mountains) (Bazyluk 1971, 1978), the occurrence of the praying mantis beyond the central part of Kotlina Sandomierska seemed to be almost unlikely.

Until 2002, the mantis had been recorded from 30 localities in Puszcza Sandomierska and 34 in the whole Kotlina Sandomierska (Liana, unpubl.) in which only 14 sites of this species had been known before (Bazyluk 1977). In 2003, a sudden "avalanche" of new information began and by the end of 2006, the number of sites known in Poland had increased nearly seven times. The majority of newly discovered sites appear to have only become inhabited recently by migration because they occurred in areas which, prior to that, had been explored intensively enough.

Apparently, an investigation of the scope and factors of this rapid expansion was necessary because $M$. religiosa was a protected species and had earlier been considered to be critically threatened. Moreover, this gave a new chance for studying the phenomena associated with area dynamics, especially interesting in the case of a species occurring at the limit of its total range. Mantis religiosa is a highly thermophilous insect and may serve as a model example of the impact of global warming on the distribution of animals.

The goal of this paper is to present the results of the research on the current distribution and environmental requirements of Mantis religiosa in Poland. All data gathered during the study, historical data (up to the 1990s) as well as new records (since the 1990s), both published by other authors and available from other sources are listed and analysed. The analysis forms a basis for discussion of distribution dynamics of this species in Central Europe.

\section{AREA AND METHODS}

For several decades, the central part of Kotlina Sandomierska - which contains Puszcza Sandomierska and the western part of Lasy Janowskie - was the only area in which the praying mantis was known to occur in Poland. Therefore, it is mainly this area, together with its immediate environs, that was taken into account while planning the research. Apart from that, an inspection of all historical localities and of the adjacent areas (if necessary) was considered too. As early as 2004 , it was necessary to extend the investigations to cover the entire eastern part of Wyżyna Malopolska as well as Wyżyna Lubelska, Pogórze Karpackie and Beskid Niski because the mantis was newly reported from these regions. In subsequent years, the author has tried to verify all new information, which resulted in further extension of the research area. The final list of the physiographic units explored is given below with their Polish name after Kondracki (1988 and later works) whose regionalization is commonly accepted by Polish geographers (Richling \& Ostaszewska 2006) ${ }^{1}$.

1. Kotlina Sandomierska $[512.4]^{2}$ (the Sandomierz Basin) including: Plaskowyż Tarnowski [512.43] (the Tarnów Plateau: vicinity of Przeclaw and Niedomice); Równina Tarnobrzeska [512.45] (the Tarnobrzeg Plain) and Plaskowyż Kolbuszowski [512.48] (the Kolbuszowa Plateau) - these regions contain the forest named Puszcza Sandomierska; Równina Bilgorajska [512.47] (the Bilgoraj Plain) with two great forest areas: Lasy Janowskie and Puszcza Solska; Plaskowyż Tarnogrodzki [512.49] (the Tarnogród Plateau) with western parts of Puszcza Solska and Lasy Sieniawskie;

2. Roztocze [343.2]: Roztocze Zachodnie [343.21] (the Western Roztocze) and Środkowe [343.22] (the Central Roztocze, with special account of the transitional zone to the Kotlina Sandomierska);

3. Wyżyna Lubelska [341.1] (the Lubelska Upland) - northern and western parts;

\footnotetext{
${ }^{1}$ The area of investigations includes parts of following administrative units: Podkarpackie, Lubelskie, Swiętokrzyskie and Mazowieckie voivodeships.

${ }^{2}$ In the square bracket they are given numbers of physiographic units after Kondracki; see:

http:/pl.wikipedia.org/wiki/Regionalizacja_fizycznogeograficzna_Polski
} 
4. Wyżyna Malopolska [342] (the Małopolska Upland): Garb Pińczowski [342.27], Niecka Połaniecka [342.28], Niecka Solecka [342.26], Płaskowyż Suchedniowski [342.31],. Pogórze Szydłowieckie [342.37], Przedgórze Ilżeckie [342.33], Wyżyna Sandomierska [342.36];

5. Nizina Mazowiecka: Nizina Pólnocnomazowiecka [318.6] (the North Mazovian Lowland), Nizina Środkowomazowiecka [318.7] (the Central Mazovian Lowland), Wzniesienia Poludniowomazowieckie [318.8] (the South Mazovian elevation);

6. Nizina Poludniowopodlaska [318.9] (the South Podlasie Lowland);

7. Polesie [845];

8. Karpaty Zachodnie [512, 513] (the West Carpathians): Pogórze Środkowobeskidzkie [513.6], Beskid Sąecki [513.54], Beskid Niski [513.71].

The exploration of Nizina Mazowiecka in search of the praying mantis was particularly intense because both of its previous occurrence in this region and the expansion of Phaneroptera falcata (Poda). This latter is a bush-cricket very often co-occurring with $M$. religiosa in Poland and it has recently been recorded as expansive in this area. The prospecting for Ph. falcata in Pojezierze Mazurskie became also an occasion to search of $M$. religiosa in this mesoregion. Due to the newly recorded occurrence of the praying mantis in Polish Carpathians, the explorations also covered several sites in northern Slovakia, adjacent to the Polish Beskid Niski, from where some new records have been published by Thomka (2003). Research in Bieszczady and Pogórze Przemyskie was, unfortunately, not conducted although the mantis was found there in 2003. Not long ago the mantis was also recorded from the Poloniny National Park adjacent in northern Slovakia to the Bieszczadzki National Park (Krištin \& Mihál 2000).

The distribution of all localities examined in 1995-2006 and all known sites of Mantis religiosa are presented in several maps (Figs 1-3). The highest concentration of the localities in the central part of Kotlina Sandomierska results from a decision concerning the strategy and scope of the research which would allow the goal to be achieved. The starting points for the research were the historical data (presented below), author's experience gathered from research conducted in the other regions, and a hypothesis, according to which central part of Kotlina Sandomierska was the main and, sometimes, the only refuge of this species in Poland.

At the earlier stage, i.e. in the first half of the 1990s, traditional methods of search for Orthoptera were used (watching and entomological net sweeping). In 1997-2002, faunistic research conducted by teams of scientists was initiated and, as a result, traps of various types were installed in many sites of Puszcza Sandomierska. They included Moericke traps, Malaise traps, Barber traps and, for some period, light traps. At the beginning of 2003, a custom-made M. religiosa questionnaire was sent out to eight forest inspectorates in Puszcza Sandomierska. The influx of survey information about the localities lasted until 2004. Wherever possible, these data were verified in the field.

In 2004, M. religiosa became the main subject of the research and, starting from that date, the search for the species was conducted most often along the transects of a total length of about $1.5-2 \mathrm{~km}$ within the period of $30 \mathrm{~min}$. approximately (similar method was used by Berg $\&$ Keller 2004 and others). Additionally, sweeping with entomological net was used to flush out or catch the nymphs and adult specimens.

Search for the oothecae proved to be a very effective method of discovering new localities of the praying mantis, and it can be useful for estimation mantis population size (Schoppmann 1989). The oothecae are most often deposited on plants near the ground or a few centimetres over it and are relatively easy to find. Even a single ootheca, especially when sticking to the ground, is evidence of earlier presence of at least one female of M. religiosa in a given locality, and a greater number of dispersed oothecae is a proof of the existence of a population. They may form a basis for a rough estimate of the size and development tendencies of the population. Autumn is the best season for searching and counting the oothecae. 


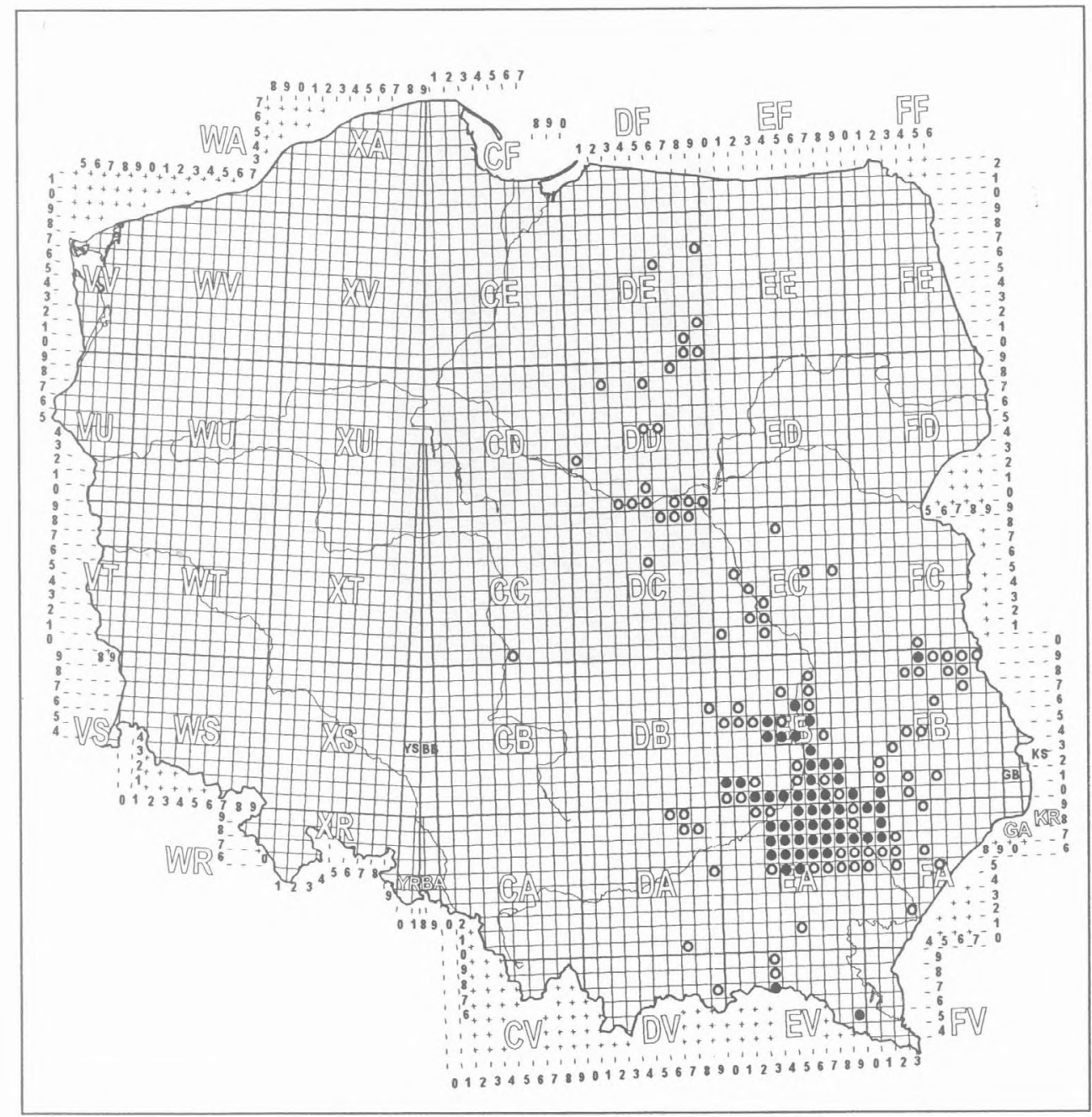

Fig. 1. Area of author's investigations in 1995-2006; full circles - presence of Mantis sites; empty circles - absence of Mantis sites (UTM grid, $10 \times 10 \mathrm{~km}$ squares).

In a few localities in Puszcza Sandomierska a method of quadrates has been used for these researches. It consisted in accurate counting of all specimens and oothecae of the praying mantis. A number of plots were selected whose area totalled at least 1 are $\left(100 \mathrm{~m}^{2}\right)$ or a multiple of this unit. In these plots adult specimens, nymphs and oothecae, were counted several times during a given season. This procedure was applied for a few years and consisted in searching every square metre of the area. The localities included: Kosowy forest division 262 near Mielec, Niwiska Podkościele, and Pniaki Wilcza Wola near Kolbuszowa and Rudnik Doliny. The count results were used to estimate the size of local populations, seasonal dynamics, and the roles of specific reducing factors, as well to gain better knowledge concerning phenology of the species in the current climatic conditions. The oothecae collected in the field were bred for several years to obtain information on potential reproductive success of the population as well as to find out to what extent it may be reduced by embryonic stage parasites. 


\section{RESULTS}

\section{Recapitulation of historical data}

Papers by Wladyslaw Bazyluk contain almost all historical data concerning the occurrence of Mantis religiosa in Poland. In his earlier papers, information was very scattered, whereas the monographs (Bazyluk 1976, 1977: map) contain information much generalized and oversimplified. A complete list of the material collected in Poland until the end of the 1950s and kept in national collections was given together with the description of a subspecies $M$. religiosa polonica Baz. (Bazyluk 1960) (including the type series of 62 specimens).

All available historical data are presented in Table 1. They include the material collected until the beginning of the 1990s and are listed in chronological order. However, they are presented according to the date of records (not publication) in specific localities. The papers containing information from different periods may, therefore, be cited several times. Such an approach has also resulted in listing two localities as historical from which the species was recorded in the 1960s without any later confirmation and which were published only 30 years later by Witkowski (1992).

Table 1. Chronological survey of historical data; abbreviations: For. - forestry, Div. - forest division.

\begin{tabular}{|c|c|c|c|c|c|c|}
\hline No. & Locality & Date & $\begin{array}{l}\text { UTM } \\
\text { gride }\end{array}$ & Environment & Source of data & Author's notes \\
\hline 1 & 2 & 3 & 4 & 5 & 6 & 7 \\
\hline 1 & Warszawa & $\begin{array}{l}\text { XVIII } \\
\text { century }\end{array}$ & $\mathrm{DC} / \mathrm{EC}$ & $\begin{array}{l}\text { surroundings } \\
\text { of city }\end{array}$ & $\begin{array}{l}\text { Perthées mscr } \\
\text { around } 1785\end{array}$ & "n'est pas rare" \\
\hline 2 & Wrocław & 1895 & $\mathrm{XS}$ & centre of city & Dietl 1895 & $\begin{array}{l}\text { „aus Menadokaffee } \\
\text { nebst...Bohnen” }\end{array}$ \\
\hline 3 & Chorzów & $\begin{array}{l}\text { before } \\
1920\end{array}$ & CA57 & $\begin{array}{l}\text { surroundings } \\
\text { of city }\end{array}$ & Pax 1920 & $\begin{array}{l}\text { "Möglichkeit einer } \\
\text { passiven Verschleppung" }\end{array}$ \\
\hline 4 & $\begin{array}{l}\text { For. Borów, Div. } \\
\text { Dzierzkowice }\end{array}$ & 1923 & EB62 & & Bazyluk 1947 & $\begin{array}{l}\text { specimen in coll. MNZ in } \\
\text { Warszawa (before 1944) }\end{array}$ \\
\hline 5 & Rudnik on the San river & 1925 & EA88 & forest & Prüffer 1955 & $\begin{array}{l}\text { a state of increase } \\
\text { Kozikowski A., inf. }\end{array}$ \\
\hline 6 & $\begin{array}{l}\text { The Sandomierz Forest, } \\
\text { For. Div. Nisko }\end{array}$ & 1927 & EA 89 & forest & Bałut 1953 & $ð ㅇ$, R. Janicki coll. \\
\hline 7 & $\begin{array}{l}\text { The Sandomierz Forest, } \\
\text { For. Nisko }\end{array}$ & $1936-1939$ & EA 89 & forest & Bałut 1953 & $\begin{array}{l}\text { several times, } \\
\text { Czarnowski M. }\end{array}$ \\
\hline 8 & $\begin{array}{l}\text { Tarnowska Wola } \\
\text { (surroundings) }\end{array}$ & 1938 & EA58 & $\begin{array}{l}\text { pinewood, } \\
\text { heath }\end{array}$ & $\begin{array}{l}\text { Karczewski } \\
1955\end{array}$ & $\begin{array}{l}\text { several specimens - it } \\
\text { wasn't a rarity }\end{array}$ \\
\hline 9 & Krzątka - Wola Tarnowska & 1938 & EA58 & heath & Bazyluk 1960 & J. Karczewski leg. \\
\hline 10 & $\begin{array}{l}\text { Forestry Brody, For. Div. } \\
\text { Lipa }\end{array}$ & 1938 & $?$ & forest & Michalski 1959 & $\begin{array}{l}5 \text { specimens, Bojarski } Z \text {. } \\
\text { coll. }\end{array}$ \\
\hline 11 & $\begin{array}{l}\text { Forestry Lipa, Zamoyski } \\
\text { Entail }\end{array}$ & 1938 & $?$ & forest & Bazyluk 1947 & as above \\
\hline 12 & "Zamojszczyzna" & before 1946 & $?$ & & $\begin{array}{l}\text { Skuratowicz } \\
1946\end{array}$ & \\
\hline 13 & $\begin{array}{l}\text { Forest to the south-west of } \\
\text { Nisko }\end{array}$ & $30 \mathrm{~s}$ of 20 th & EA89 & & Michalski 1959 & $\begin{array}{l}\text { one specimen found by } \\
\text { prof. Suchecki }\end{array}$ \\
\hline 14 & $\begin{array}{l}\text { The Sandomierz Forest, } \\
\text { For. Nisko }\end{array}$ & $1946-1950$ & EA 89 & forest & Bałut 1953 & \\
\hline 15 & Surroundings of Biłgoraj & & FB20 & & Bałut 1953 & \\
\hline 16 & Surroundings of Skarżysko & & DB96? & & Bałut 1953 & \\
\hline 17 & $\begin{array}{l}\text { Forestry Barce, For. Div. } \\
\text { Nisko }\end{array}$ & 1950 & EA89? & $\begin{array}{l}\text { dune, young } \\
\text { pine growth }\end{array}$ & Bazyluk 1960 & q, Bałut S. leg. \\
\hline 18 & $\begin{array}{l}\text { Dęba Rozalin near } \\
\text { Tarnobrzeg }\end{array}$ & 1952 & EA58 & dune & Razowski 1953 & several specimens \\
\hline 19 & $\begin{array}{l}\text { Surroundings of } \\
\text { Kolbuszowa }\end{array}$ & 1953 & EA56? & & Razowski 1953 & one specimen \\
\hline
\end{tabular}




\begin{tabular}{|c|c|c|c|c|c|c|}
\hline 1 & 2 & 3 & 4 & 5 & 6 & 7 \\
\hline 20 & $\begin{array}{l}\text { Garbatka near railway } \\
\text { station }\end{array}$ & 1953 & & & Michalski 1959 & $\begin{array}{l}\text { specimen killed by a bird, } \\
\text { Kowalczuk info } 1953\end{array}$ \\
\hline 21 & $\begin{array}{l}\text { Dęba Rozalin to the north } \\
\text { of station }\end{array}$ & 1953 & EA58? & $\begin{array}{l}\text { pine forest } \\
\text { margin }\end{array}$ & Bazyluk 1957 & $\begin{array}{l}\text { imago and nymph on } \\
\text { Vaccinium }\end{array}$ \\
\hline 22 & $\begin{array}{l}\text { Krzatka (surroundings of } \\
\text { Tarnowska Wola }\end{array}$ & 1953 & EA68 & heath & Bazyluk 1957 & imagines, nymphe \\
\hline 23 & For. Div. Modliborzyce & 1954 & EB91? & & Michalski 1959 & \\
\hline 24 & $\begin{array}{l}\text { Surroundings of } \\
\text { Kolbuszowa }\end{array}$ & $\begin{array}{l}\text { before } \\
1955\end{array}$ & EA56? & & Bazyluk 1956 & \\
\hline 25 & Garbatka near Kozienice & $\begin{array}{l}\text { before } \\
1955\end{array}$ & & & Bazyluk 1956 & \\
\hline 26 & $\begin{array}{l}\text { vicinity of Nisko to the } \\
\text { west of town }\end{array}$ & 1955 & EA79 & $\begin{array}{l}\text { logging area in } \\
\text { a pine forest }\end{array}$ & Bazyluk 1957 & not abundant \\
\hline 27 & $\begin{array}{l}\text { vicinity Mielec to the east } \\
\text { of town }\end{array}$ & 1955 & EA36 & $\begin{array}{l}\text { heath in a pine } \\
\text { forest }\end{array}$ & Bazyluk 1957 & \\
\hline 28 & $\begin{array}{l}\text { Mielec/Kolbuszowa } \\
\text { between districts }\end{array}$ & 1955 & EA46 & & Bazyluk 1957 & \\
\hline 29 & $\begin{array}{l}\text { Rozwadów [today Stalowa } \\
\text { Wola-Rozwadów] }\end{array}$ & $\begin{array}{l}1956, \\
1957,1958\end{array}$ & EB70 & $\begin{array}{l}\text { heath in a pine } \\
\text { forest }\end{array}$ & Bazyluk 1960 & $\delta \delta, 90$, oothecae \\
\hline 30 & Stalowa Wola & 1956,1958 & EB70 & $\begin{array}{l}\text { heath in a pine } \\
\text { forest }\end{array}$ & Bazyluk 1960 & $30,9 q$, oothecae \\
\hline 31 & $\begin{array}{l}\text { Góry Wysokie near } \\
\text { Sandomierz }\end{array}$ & 1956,1958 & EB52 & Polana & Bazyluk 1960 & $\partial \partial$, 우, oothecae \\
\hline 32 & $\begin{array}{l}\text { For. Kochany, Div. } 68 \text {, to } \\
\text { the NE of Rozwadów }\end{array}$ & 1958 & EB81? & $\begin{array}{l}\text { heath on the } \\
\text { hill within } \\
\text { marhes }\end{array}$ & Michalski 1959 & 80 , ootheca \\
\hline 33 & Zaklików & 1968 & EB72 & $\begin{array}{l}\text { Arctostaphyllo } \\
\text {-Callunetum }\end{array}$ & Cmoluch 1971 & $\partial$ o \\
\hline 34 & $\begin{array}{l}\text { Chotel Czerwony - Niecka } \\
\text { Nidziańska }\end{array}$ & 1968 & DA78 & steppe reserve & $\begin{array}{l}\text { Witkowski } \\
1992\end{array}$ & $\begin{array}{l}\text { one specimen, Petryszak } \\
\text { inf. }\end{array}$ \\
\hline 35 & Pieniny, Nowa Góra & 1968 & DV57 & $\begin{array}{l}\text { mountain } \\
\text { meadow }\end{array}$ & $\begin{array}{l}\text { Witkowski } \\
1992\end{array}$ & $\begin{array}{l}\text { several specimens, Oleś } \\
\text { inf. }\end{array}$ \\
\hline
\end{tabular}

A manuscript by Karol Perthées contained information about the occurrence of the praying mantis near Warsaw in the 18th century. This information was published for the first time by Waga (1843). Karol Perthées was the king's geographer of Stanisław August Poniatowski. He was the author of very precise maps of provinces (at that time) drawn on a scale of 1:225000. Perthées also had profound entomological knowledge which allowed him to professionally collect and describe insects (Pawłowski 2003). In relation to the praying mantis, Perthées used the expression "n'est pas rare", so there was no doubt that he must have observed it near Warsaw several times. On the basis of the measurements of a female kept in Perthées's collection and presented in his manuscript, Bazyluk (1977) considered it as belonging to the subspecies $M$. religiosa polonica. The fact that Perthées did not provide the exact name of the locality confirms the hypothesis that he must have treated the praying mantis as an ordinary element of the local fauna.

There is no reliable information about the occurrence of $M$. religiosa in Poland during the 19 th century. The only record from that period (Dietl 1895) concerned a specimen introduced to Wroclaw with the so called colonial food commodities. We have quite a lot of information dating from the first half of the 20th century but most of it was published as late as the $1950 \mathrm{~s}$, when nearly $50 \%$ of all historical localities were recorded (Table 1). It is noteworthy that the vast majority of the localities (12) were located in a central part of Kotlina Sandomierska and some of them were mentioned by different authors. A very general term "Zamojszczyzna" or „Ordynacja Zamoyskich" (Skuratowicz 1946, Bazyluk 1947) referred to the western part of Puszcza Solska and Lasy Janowskie but not to Roztocze, as it was wrongly interpreted 
afterwards. Balut (1953) stated that the mantis occurred in the environs of Skarżysko and Bilgoraj but he did not give any sources of information which was rather strange. In the case of Puszcza Sandomierska, where he observed this species himself, not only did he name the localities but also give the names of other observers and observation dates ${ }^{3}$.

All the historical localities of the praying mantis (or, for lack of precise information about their location, the areas in which they probably were) have also been explored afterwards. In Mazovia, the scientists searched for the praying mantis many times, especially in the vicinity of Warsaw, in Puszcza Kozienicka (the Kozienice Forest) near Garbatka, in Góry Wysokie near Sandomierz, and in the vicinity of Skarżysko Kamienna. The prospecting for the praying mantis in these localities was also repeated within the scope of the newest stage of the research.

\section{Current data}

The particular data collected by author research in 1995-2006 are presented in a synthetic tabular form which includes among other information on geographical situation of each $M$. religiosa site, habitat, developmental stage of Mantis, and anticipated trend of given population (Table 2). The trend is estimated on a basis of such information as an age of a site and its history (concluded on the basis of the present character of vegetation, map records and information from the owners or land users), characteristics of the population itself (number of observed adults and other developmental stages, their condition, etc.), as well as existing and potential danger. It must be added that all imagines recorded in Table 2 have been classified as M. religiosa polonica BAZ. with the exception of a female from Cisna (Bieszczady Mts.).

Table 2. The sites of Mantis religiosa populations recorded in 1995-2006. Explanations of symbols and abbreviations: $\infty$-durable stable population; $\infty$ and $\leftrightarrow-$ durable mobile population; $\leftrightarrow$ migrant population; $\uparrow$ - pioneer population; $\downarrow$ vanishing population; $\uparrow$ - ephemeral population (or single migrant only); ? - trend of population difficult to estimate; intro - introduction; $\not$-female(s); $\not$ - male(s); ootheca - ootheca(e); for. - forest section;

\begin{tabular}{|c|c|c|c|c|c|c|c|}
\hline No & Sites & UTM & GEO & $\begin{array}{c}\text { Stages } \\
\text { observed }\end{array}$ & Environment & Vegetation & Trend \\
\hline 1 & 2 & 3 & 4 & 5 & 6 & 7 & 8 \\
\hline \multicolumn{8}{|c|}{ Kotlina Sandomierska (The Sandomierska Basin) } \\
\hline 1 & $\begin{array}{l}\text { Agatówka near } \\
\text { Stalowa Wola }\end{array}$ & EB70 & $\begin{array}{l}\text { N } 50^{\circ} 37^{\prime} \\
\text { E } 22^{\circ} 01^{\prime}\end{array}$ & 9 , ootheca & $\begin{array}{l}\text { forest margin } \\
\text { housing estate }\end{array}$ & heath fragmented & $\downarrow$ \\
\hline 2 & $\begin{array}{l}\text { Alfredówka near } \\
\text { Nowa Dęba }\end{array}$ & EA59 & $\begin{array}{l}\text { N } 50^{\circ} 28^{\prime} \\
\text { E } 21^{\circ} 44^{\prime}\end{array}$ & $\varnothing$ & open meadows & wet meadow drained & $\leftrightarrow$ \\
\hline 3 & $\begin{array}{l}\text { Blizna I near } \\
\text { Sędziszów Młp. }\end{array}$ & EA46 & $\begin{array}{l}\text { N } 50^{\circ} 11^{\prime} \\
\text { E } 21^{\circ} 36^{\prime}\end{array}$ & $\begin{array}{l}\delta, q \\
\text { ootheca }\end{array}$ & $\begin{array}{l}\text { large clearing, } \\
\text { fallow lands }\end{array}$ & $\begin{array}{l}\text { dry sandy grassland } \\
\text { Corynophoretum }\end{array}$ & $\leftrightarrow$ \\
\hline 4 & $\begin{array}{l}\text { Blizna II near } \\
\text { Sędziszów Młp }\end{array}$ & $\begin{array}{l}\text { EA } \\
46\end{array}$ & $\begin{array}{l}\text { N } 50^{\circ} 12^{\prime} \\
\text { E } 21^{\circ} 36^{\prime}\end{array}$ & $\delta$ & $\begin{array}{l}\text { large clearing, } \\
\text { fallow lands }\end{array}$ & $\begin{array}{l}\text { dry sandy grassland } \\
\text { Corynophoretum }\end{array}$ & $\leftrightarrow$ \\
\hline 5 & Bojanów I, Lysa Góra & EA68 & $\begin{array}{l}\text { N } 50^{\circ} 25^{\prime} \\
\text { E } 21^{\circ} 57^{\prime}\end{array}$ & q, ootheca & $\begin{array}{l}\text { fallow lands at the } \\
\text { forest margin }\end{array}$ & $\begin{array}{l}\text { dry sandy grassland } \\
\text { Corynophoretum }\end{array}$ & $\leftrightarrow$ \\
\hline 6 & $\begin{array}{l}\text { Bojanow II by the } \\
\text { military training ground }\end{array}$ & EA68 & $\begin{array}{l}\text { N } 50^{\circ} 26^{\prime} \\
\text { E } 21^{\circ} 54^{\prime}\end{array}$ & 9, ootheca & $\begin{array}{l}\text { forest } \\
\text { margin/wastland }\end{array}$ & $\begin{array}{l}\text { heath/dry sandy grass- } \\
\text { land Corynophoretum }\end{array}$ & $\infty \leftrightarrow$ \\
\hline 7 & Borów near Radomyśl & EB63 & $\begin{array}{l}\text { N } 50^{\circ} 48^{\prime} \\
\text { E } 21^{\circ} 55^{\prime}\end{array}$ & ootheca & small clearings & $\begin{array}{l}\text { dry sandy grassland } \\
\text { Corynophoretum }\end{array}$ & $\stackrel{\infty}{\leftrightarrow}$ \\
\hline 8 & $\begin{array}{l}\text { Brzostowa Góra near } \\
\text { Nowa Dęba }\end{array}$ & EA58 & $\begin{array}{l}\text { N } 50^{\circ} 23^{\prime} \\
\text { E } 21^{\circ} 47^{\prime}\end{array}$ & $\begin{array}{l}\delta, q \\
\text { ootheca }\end{array}$ & $\begin{array}{l}\text { ecotone: sand } \\
\text { dune/meadow }\end{array}$ & $\begin{array}{l}\text { dry sandy grassland/ } \\
\text { meadow }\end{array}$ & $\leftrightarrow$ \\
\hline 9 & Bukie near Tarnobrzeg & EA59 & $\begin{array}{l}\text { N } 50^{\circ} 31^{\prime} \\
\text { E } 21^{\circ} 46^{\prime}\end{array}$ & ootheca & $\begin{array}{l}\text { ecotone: forest } \\
\text { margin } / \text { meadow }\end{array}$ & wet hay-meadow drained & $\uparrow$ \\
\hline 10 & $\begin{array}{l}\text { Bukowiec near } \\
\text { Kolbuszowa }\end{array}$ & EA56 & $\begin{array}{l}\text { N } 50^{\circ} 13^{\prime} \\
\text { E } 21^{\circ} 46^{\prime}\end{array}$ & ootheca & $\begin{array}{l}\text { forest } \\
\text { margin/wasteland }\end{array}$ & $\begin{array}{l}\text { dry sandy grassland } \\
\text { Corynophoretum }\end{array}$ & $\uparrow$ \\
\hline
\end{tabular}

\footnotetext{
${ }^{3}$ Bałut (1953) recorded also Lódź but he mentioned this locality as "very uncertain". This information, repeated by many authors, was explained as wrong by Bazyluk $(1960,1976)$.
} 


\begin{tabular}{|c|c|c|c|c|c|c|c|}
\hline 1 & 2 & 3 & 4 & 5 & 6 & 7 & 8 \\
\hline 11 & $\begin{array}{l}\text { Burdze I near } \\
\text { Przyszów }\end{array}$ & EA79 & $\begin{array}{l}\text { N } 50^{\circ} 30^{\prime} \\
\text { E } 21^{\circ} 59^{\prime}\end{array}$ & 9, ootheca & large clearing & $\begin{array}{l}\text { heath/dry sandy grass- } \\
\text { land Corynophoretum }\end{array}$ & $\begin{array}{l}\infty \\
\leftrightarrow\end{array}$ \\
\hline 12 & $\begin{array}{l}\text { Burdze II near } \\
\text { Przyszów }\end{array}$ & EA69 & $\begin{array}{l}\text { N } 50^{\circ} 30^{\prime} \\
\text { E } 21^{\circ} 58^{\prime}\end{array}$ & ootheca & small clearings & $\begin{array}{l}\text { dry sandy } \\
\text { grassland/ruderal }\end{array}$ & $\leftrightarrow$ \\
\hline 13 & $\begin{array}{l}\text { Burdze III near } \\
\text { Przyszów }\end{array}$ & EA69 & $\begin{array}{l}\text { N } 50^{\circ} 31^{\prime} \\
\text { E } 21^{\circ} 58^{\prime}\end{array}$ & 9, ootheca & $\begin{array}{l}\text { fellow lands on } \\
\text { the Leg river }\end{array}$ & $\begin{array}{l}\text { dry sandy grassland } \\
\text { Corynophoretum }\end{array}$ & $\stackrel{\infty}{\leftrightarrow}$ \\
\hline 14 & $\begin{array}{l}\text { Burdze IV near } \\
\text { Przyszów }\end{array}$ & EA69 & $\begin{array}{l}\text { N } 50^{\circ} 31^{\prime} \\
\text { E } 21^{\circ} 58^{\prime}\end{array}$ & ootheca & $\begin{array}{l}\text { forest margin on } \\
\text { the slope of hill }\end{array}$ & $\begin{array}{l}\text { heath/dry sandy grass- } \\
\text { land Corynophoretum }\end{array}$ & $\stackrel{\infty}{\leftrightarrow}$ \\
\hline 15 & $\begin{array}{l}\text { Burdze V near } \\
\text { Przyszów }\end{array}$ & EA79 & $\begin{array}{l}\text { N } 50^{\circ} 31^{\prime} \\
\text { E } 21^{\circ} 59^{\prime}\end{array}$ & 9 , ootheca & $\begin{array}{l}\text { pinewood, forest } \\
\text { track }\end{array}$ & $\begin{array}{l}\text { heath/dry sandy grass- } \\
\text { land Corynophoretum }\end{array}$ & $\stackrel{\infty}{\leftrightarrow}$ \\
\hline 16 & $\begin{array}{l}\text { Charzewice near } \\
\text { Stalowa Wola }\end{array}$ & EB70 & $\begin{array}{l}\text { N } 50^{\circ} 36^{\prime} \\
\text { E } 22^{\circ} 01^{\prime}\end{array}$ & $\begin{array}{l}\text { A, } 9 \text {, } \\
\text { ootheca }\end{array}$ & $\begin{array}{l}\text { ecotone; forest } \\
\text { edge/wastland }\end{array}$ & $\begin{array}{l}\text { dry sandy grassland/ ruderal } \\
\text { (border of housing estate) }\end{array}$ & $\downarrow$ \\
\hline 17 & Ciemny Kąt for. 242 & EB70 & $\begin{array}{l}\text { N } 50^{\circ} 33^{\prime} \\
\text { E } 22^{\circ} 01\end{array}$ & ootheca & $\begin{array}{l}\text { ecotone; forest } \\
\text { clearing }\end{array}$ & heath and forestry crops & $\stackrel{\infty}{\leftrightarrow}$ \\
\hline 18 & $\begin{array}{l}\text { Cierpisz near } \\
\text { Kolbuszowa }\end{array}$ & EA55 & $\begin{array}{l}\text { N } 50^{\circ} 08^{\prime} \\
\text { E } 21^{\circ} 43^{\prime}\end{array}$ & ootheca & open; fallow land & dry sandy grassland & $\uparrow$ \\
\hline 19 & $\begin{array}{l}\text { Cietrzewiec I Zamek } \\
\text { (Castle) }\end{array}$ & EA69 & $\begin{array}{l}\mathrm{N} 50^{\circ} 26^{\prime} \\
\mathrm{E} 21^{\circ} 50^{\prime}\end{array}$ & $\begin{array}{l}\hat{0},+, \\
\text { ootheca }\end{array}$ & $\begin{array}{l}\text { open; military } \\
\text { training ground }\end{array}$ & $\begin{array}{l}\text { heath/dry grassland } \\
\text { Corynophoretum patches }\end{array}$ & $\infty$ \\
\hline 20 & $\begin{array}{l}\text { Cietrzewiec Wyspa } \\
\text { („Island") }\end{array}$ & EA69 & $\begin{array}{l}\text { N } 50^{\circ} 27^{\prime} \\
\text { E } 21^{\circ} 51^{\prime}\end{array}$ & 9 , ootheca & $\begin{array}{l}\text { open; military } \\
\text { training ground }\end{array}$ & $\begin{array}{l}\text { heath/dry grassland } \\
\text { Corynophoretum patches }\end{array}$ & $\infty$ \\
\hline 21 & $\begin{array}{l}\text { Cietrzewiec III } \\
\text { Wzgórze (hill) } 211\end{array}$ & EA69 & $\begin{array}{l}\text { N } 50^{\circ} 27^{\prime} \\
\text { E } 21^{\circ} 51^{\prime}\end{array}$ & $\begin{array}{l}\delta, 9 \\
\text { ootheca }\end{array}$ & $\begin{array}{l}\text { open; military } \\
\text { training ground }\end{array}$ & $\begin{array}{l}\text { heath/dry grassland } \\
\text { Corynophoretum patches }\end{array}$ & $\infty$ \\
\hline 22 & $\begin{array}{l}\text { Cmolas near } \\
\text { Kolbuszowa }\end{array}$ & EA57 & $\begin{array}{l}\text { N } 50^{\circ} 19^{\prime} \\
\text { E } 21^{\circ} 44^{\prime}\end{array}$ & ootheca & $\begin{array}{l}\text { ecotone; forest } \\
\text { edge/wastland }\end{array}$ & $\begin{array}{l}\text { dry sandy grassland } \\
\text { ruderal }\end{array}$ & $\uparrow$ \\
\hline 23 & Czarna Sędziszowska & EA55 & $\begin{array}{l}\text { N } 50^{\circ} 08^{\prime} \\
\text { E } 21^{\circ} 45^{\circ}\end{array}$ & ootheca & $\begin{array}{l}\text { ecotone; forest } \\
\text { edge/fallow }\end{array}$ & $\begin{array}{l}\text { dry sandy grassland } \\
\text { meadow with pine crops }\end{array}$ & $\uparrow$ \\
\hline 24 & $\begin{array}{l}\text { Czarny Las L. near } \\
\text { Rudnik }\end{array}$ & EA88 & $\begin{array}{l}\text { N } 56^{\circ} 26^{\prime} \\
\text { E } 22^{\circ} 12^{\prime}\end{array}$ & imago & $\begin{array}{l}\text { ecotone; logging } \\
\text { area }\end{array}$ & & $\uparrow$ \\
\hline 25 & $\begin{array}{l}\text { Dąbrowica Duża near } \\
\text { Leżajsk }\end{array}$ & FA17 & $\begin{array}{l}\mathrm{N} 50^{\circ} 18^{\prime} \\
\mathrm{E} 22^{\circ} 33^{\prime}\end{array}$ & ootheca & $\begin{array}{l}\text { ecotone; forest } \\
\text { edge/fallow }\end{array}$ & $\begin{array}{l}\text { dry sandy } \\
\text { grassland/ruderal }\end{array}$ & $\uparrow$ \\
\hline 26 & $\begin{array}{l}\text { Dąbrowskie Góry near } \\
\text { Nowa Dęba }\end{array}$ & EA69 & $\begin{array}{l}\text { N } 50^{\circ} 28^{\prime} \\
\text { E } 21^{\circ} 52^{\prime}\end{array}$ & imago & $\begin{array}{l}\text { open; military } \\
\text { training ground }\end{array}$ & $\begin{array}{l}\text { heath and dry sandy } \\
\text { grassland Corynophoretum }\end{array}$ & $\infty$ \\
\hline 27 & $\begin{array}{l}\text { Dęba Rozalin near } \\
\text { Nowa Dęba }\end{array}$ & EA58 & $\begin{array}{l}\text { N } 50^{\circ} 26^{\prime} \\
\text { E } 21^{\circ} 42^{\prime}\end{array}$ & 9 , ootheca & $\begin{array}{l}\text { ecotone; forest } \\
\text { edge/wastland }\end{array}$ & $\begin{array}{l}\text { dry sandy grassland /- } \\
\text { ruderal }\end{array}$ & $\stackrel{\infty}{\leftrightarrow}$ \\
\hline 28 & $\begin{array}{l}\text { Dobrynin near } \\
\text { Tuszyma }\end{array}$ & EA36 & $\begin{array}{l}\text { N } 50^{\circ} 13^{\prime} \\
\text { E } 21^{\circ} 32^{\prime}\end{array}$ & ootheca & $\begin{array}{l}\text { ecotone; forest } \\
\text { edge/fallow }\end{array}$ & $\begin{array}{l}\text { dry sandy grassland } \\
\text { Corynophoretum }\end{array}$ & $\uparrow$ \\
\hline 29 & $\begin{array}{l}\text { Gómo near Sokołów } \\
\text { Matopolski }\end{array}$ & EA87 & $\begin{array}{l}\text { N } 50^{\circ} 18^{\prime} \\
\text { E } 22^{\circ} 07^{\prime}\end{array}$ & 8 & building ad lucem & $\begin{array}{l}\text { [sandy fallow lands in } \\
\text { immediate vicinity] }\end{array}$ & $\uparrow$ \\
\hline 30 & $\begin{array}{l}\text { Grębów Stacja near } \\
\text { Tarnobrzeg }\end{array}$ & EB50 & $\begin{array}{l}\text { N } 50^{\circ} 36^{\prime} \\
\text { E } 21^{\circ} 51^{\prime}\end{array}$ & ootheca & $\begin{array}{l}\text { ecotone; forest } \\
\text { edge/fallows }\end{array}$ & $\begin{array}{l}\text { dry sandy grassland } \\
\text { Corynophoretum }\end{array}$ & $\uparrow$ \\
\hline 31 & Grębów Zajezierze & EB60 & $\begin{array}{l}\mathrm{N} 50^{\circ} 34^{\prime} \\
\mathrm{E} 21^{\circ} 52^{\prime}\end{array}$ & $\begin{array}{l}3, q, \\
\text { ootheca }\end{array}$ & open fallow lands & $\begin{array}{l}\text { dry sandy grassland } \\
\text { ruderal }\end{array}$ & $\uparrow$ \\
\hline 32 & $\begin{array}{l}\text { Groble Katy near } \\
\text { Rudnik }\end{array}$ & EA88 & $\begin{array}{l}\text { N } 50^{\circ} 22^{\prime} \\
\text { E } 22^{\circ} 12^{\prime}\end{array}$ & $\begin{array}{l}\curvearrowright, q \\
\text { ootheca }\end{array}$ & $\begin{array}{l}\text { ecotone; forest } \\
\text { edge/fallow }\end{array}$ & $\begin{array}{l}\text { dry sandy grassland } \\
\text { /meadow }\end{array}$ & $\uparrow$ \\
\hline 33 & $\begin{array}{l}\text { Grochowe I near } \\
\text { Mielec }\end{array}$ & EA37 & $\begin{array}{l}\text { N } 50^{\circ} 20^{\prime} \\
\text { E } 21^{\circ} 32^{\prime}\end{array}$ & ootheca & $\begin{array}{l}\text { ecotone; forest } \\
\text { edge/meadow }\end{array}$ & Calluno-Nardetum & $\leftrightarrow$ \\
\hline 34 & $\begin{array}{l}\text { Grochowe II near } \\
\text { Mielec }\end{array}$ & EA37 & $\begin{array}{l}\text { N } 50^{\circ} 20^{\prime} \\
\text { E } 21^{\circ} 31^{\prime}\end{array}$ & $\begin{array}{l}\delta, q \\
\text { ootheca }\end{array}$ & open meadow & $\begin{array}{l}\text { wet meadow with Juncus } \\
\text { effuses }\end{array}$ & $\uparrow$ \\
\hline 35 & $\begin{array}{l}\text { Gwoździec I near } \\
\text { Wilcza Wola }\end{array}$ & EA78 & $\begin{array}{l}\text { N } 50^{\circ} 23^{\prime} \\
\text { E } 21^{\circ} 59^{\prime}\end{array}$ & q, ootheca & $\begin{array}{l}\text { ecotone; forest } \\
\text { edge/meadow }\end{array}$ & $\begin{array}{l}\text { dry sandy grassland } \\
\text { meadow }\end{array}$ & $\leftrightarrow$ \\
\hline 36 & $\begin{array}{l}\text { Gwoździec II near } \\
\text { Wilcza Wola }\end{array}$ & EA78 & $\begin{array}{l}\text { N } 50^{\circ} 23^{\prime} \\
\text { E } 21^{\circ} 59^{\prime}\end{array}$ & ootheca & $\begin{array}{l}\text { ecotone; forest } \\
\text { edge/meadows }\end{array}$ & heath $/$ meadow & $\stackrel{\infty}{\leftrightarrow}$ \\
\hline 37 & $\begin{array}{l}\text { Gwoździec III near } \\
\text { Wilcza Wola }\end{array}$ & EA78 & $\begin{array}{l}\text { N } 50^{\circ} 22^{\prime} \\
\text { E } 21^{\circ} 60^{\prime}\end{array}$ & ootheca & $\begin{array}{l}\text { ecotone; forest } \\
\text { edge/meadow }\end{array}$ & $\begin{array}{l}\text { wet meadow with Juncus } \\
\text { effuses }\end{array}$ & $\uparrow ?$ \\
\hline 38 & $\begin{array}{l}\text { Hucisko near Głogów } \\
\text { Małopolski }\end{array}$ & EA76 & $\begin{array}{l}\text { N } 50^{\circ} 13^{\prime} \\
\mathrm{E} 22^{\circ} 01^{\prime}\end{array}$ & O, ootheca & $\begin{array}{l}\text { ecotone; forest } \\
\text { edge/follows }\end{array}$ & $\begin{array}{l}\text { dry sandy grassland } \\
\text { Corynophoretum }\end{array}$ & $\uparrow$ \\
\hline
\end{tabular}

\footnotetext{
${ }^{4}$ Based on one specimen captured in the building of sanatorium by Franciszek Cepielik
} 


\begin{tabular}{|c|c|c|c|c|c|c|c|}
\hline 1 & 2 & 3 & 4 & 5 & 6 & 7 & 8 \\
\hline 39 & Huta Przedborska Kąty & EA56 & $\begin{array}{l}\text { N } 50^{\circ} 11^{\prime} \\
\text { E } 21^{\circ} 43^{\prime}\end{array}$ & $\begin{array}{l}\text { juv., d, }, \text {, } \\
\text { ootheca }\end{array}$ & $\begin{array}{l}\text { ecotone; forest } \\
\text { edge/follows }\end{array}$ & $\begin{array}{l}\text { dry sandy grassland } \\
\text { Corynophoretum }\end{array}$ & intro \\
\hline 40 & $\begin{array}{l}\text { Huta Przedborska Pod } \\
\text { Lasem }\end{array}$ & EA56 & $\begin{array}{l}\text { N } 50^{\circ} 11^{\prime} \\
\text { E } 21^{\circ} 44^{\prime}\end{array}$ & q, ootheca & $\begin{array}{l}\text { ecotone; forest } \\
\text { edge }\end{array}$ & $\begin{array}{l}\text { dry sandy grassland/ } \\
\text { segetal }\end{array}$ & $\uparrow$ \\
\hline 41 & $\begin{array}{l}\text { Hyki Dębiaki near } \\
\text { Mielec }\end{array}$ & EA37 & $\begin{array}{l}\text { N } 50^{\circ} 21^{\prime} \\
\text { E } 21^{\circ} 32^{\prime}\end{array}$ & ootheca & $\begin{array}{l}\text { ecotone; forest } \\
\text { edge }\end{array}$ & $\begin{array}{l}\text { dry sandy grassland } \\
\text { Corynophoretum }\end{array}$ & $\leftrightarrow$ \\
\hline 42 & $\begin{array}{l}\text { Imielty Lug near } \\
\text { Janów Lubelski }\end{array}$ & EB81 & $\begin{array}{l}\text { N } 50^{\circ} 40^{\prime} \\
\text { E } 22^{\circ} 13\end{array}$ & $\delta$ & large clearing & $\begin{array}{l}\text { Peatbog Ledo- } \\
\text { Sphagnetum }\end{array}$ & $\uparrow$ \\
\hline 43 & Jamnica I near Grębów & EB60 & $\begin{array}{l}\text { N } 50^{\circ} 34^{\prime} \\
\text { E } 21^{\circ} 59^{\prime}\end{array}$ & $\begin{array}{l}\text { juv., } 9, \\
\text { ootheca }\end{array}$ & $\begin{array}{l}\text { ecotone; clearing/ } \\
\text { young growth }\end{array}$ & $\begin{array}{l}\text { dry sandy grassland/ } \\
\text { young pine growth }\end{array}$ & $\stackrel{\downarrow}{2003}$ \\
\hline 44 & $\begin{array}{l}\text { Jamnica II near } \\
\text { Grębów }\end{array}$ & EB60 & $\begin{array}{l}\text { N } 50^{\circ} 35^{\prime} \\
\text { E } 21^{\circ} 58^{\prime}\end{array}$ & ootheca & $\begin{array}{l}\text { ecotone; forest } \\
\text { edge/fallow }\end{array}$ & $\begin{array}{l}\text { dry sandy grassland } \\
\text { Corynophoretum }\end{array}$ & $\uparrow$ \\
\hline 45 & Jamnica III for. Zaosie & EB60 & $\begin{array}{l}\text { N } 50^{\circ} 35^{\prime} \\
\text { E } 21^{\circ} 59^{\prime}\end{array}$ & ootheca & $\begin{array}{l}\text { zone deforested in } \\
\text { pine forest }\end{array}$ & $\begin{array}{l}\text { pinewood } \\
\text { undergrowth/dry sandy } \\
\text { grassland/ruderal }\end{array}$ & $\stackrel{\infty}{\leftrightarrow}$ \\
\hline 46 & Jelna near Leżajsk & EA97 & $\begin{array}{l}\text { N } 50^{\circ} 16^{\prime} \\
\text { E } 22^{\circ} 22^{\prime}\end{array}$ & ootheca & $\begin{array}{l}\text { ecotone; forest } \\
\text { edge/fallow }\end{array}$ & $\begin{array}{l}\text { dry sandy grassland } \\
\text { Corynophoretum }\end{array}$ & $\uparrow$ \\
\hline 47 & $\begin{array}{l}\text { Jelna (meadows) near } \\
\text { Stalowa Wola }\end{array}$ & EA79 & $\begin{array}{l}\text { N } 50^{\circ} 31^{\prime} \\
\text { E } 22^{\circ} 03\end{array}$ & $\begin{array}{l}\AA, \text { P } \\
\text { ootheca }\end{array}$ & large clearing & wet meadows drained & $\uparrow$ \\
\hline 48 & Jeziórko near Grębów & EB50 & $\begin{array}{l}\text { N } 50^{\circ} 33^{\prime} \\
\text { E } 21^{\circ} 49^{\prime}\end{array}$ & ootheca & open; fallow lands & $\begin{array}{l}\text { dry sandy grassland } \\
\text { Corynophoretum }\end{array}$ & $\uparrow$ \\
\hline 49 & Kamionka Sekwest & EA45 & $\begin{array}{l}\text { N } 50^{\circ} 08^{\prime} \\
\text { E } 21^{\circ} 39^{\prime}\end{array}$ & 9 , ootheca & $\begin{array}{l}\text { ecotone; forest } \\
\text { edge/fallow }\end{array}$ & $\begin{array}{l}\text { dry sandy grassland } \\
\text { Corynophoretum }\end{array}$ & $\uparrow$ \\
\hline 50 & $\begin{array}{l}\text { Karolówka near } \\
\text { Tarnobrzeg }\end{array}$ & EA48 & $\begin{array}{l}\text { N } 50^{\circ} 26^{\prime} \\
\text { E } 21^{\circ} 37^{\prime}\end{array}$ & ootheca & open; meadows & $\begin{array}{l}\text { wet meadow with Juncus } \\
\text { effuses }\end{array}$ & $\uparrow$ \\
\hline 51 & Kolbuszowa $^{5}$ & EA56 & $\begin{array}{l}\text { N } 50^{\circ} 14^{\prime} \\
\text { E } 21^{\circ} 45^{\prime}\end{array}$ & $q$ & $\begin{array}{l}\text { outskirts of the } \\
\text { town }\end{array}$ & sedge meadow drained & $\uparrow$ \\
\hline 52 & $\begin{array}{l}\text { Komorów near Nowa } \\
\text { Dęba }\end{array}$ & EA58 & $\begin{array}{l}\text { N } 50^{\circ} 21^{\prime} \\
\text { E } 21^{\circ} 43^{\prime}\end{array}$ & q & $\begin{array}{l}\text { ecotone; pine forest } \\
\text { margin/ grassland }\end{array}$ & $\begin{array}{l}\text { undergrowth with fern/ } \\
\text { dry sandy grassland }\end{array}$ & $\leftrightarrow$ \\
\hline 53 & $\begin{array}{l}\text { Komorów Koniec near } \\
\text { Nowa Dęba }\end{array}$ & EA58 & $\begin{array}{l}\text { N } 50^{\circ} 21^{\prime} \\
\text { E } 21^{\circ} 44^{\prime}\end{array}$ & ootheca & $\begin{array}{l}\text { open; moist } \\
\text { meadows }\end{array}$ & Calluno-Nardetum & $\leftrightarrow$ \\
\hline 54 & $\begin{array}{l}\text { Konefaly near Nowa } \\
\text { Dęba }\end{array}$ & EA68 & $\begin{array}{l}\text { N } 50^{\circ} 22^{\prime} \\
\text { E } 21^{\circ} 52^{\prime}\end{array}$ & ootheca & $\begin{array}{l}\text { ecotone; forest } \\
\text { edge/fallow }\end{array}$ & heath/dry sandy grassland & $\stackrel{\infty}{\leftrightarrow}$ \\
\hline 55 & $\begin{array}{l}\text { Korczowiska near } \\
\text { Sokolów Mlp. }\end{array}$ & EA 77 & $\begin{array}{l}\text { N } 50^{\circ} 18^{\prime} \\
\text { E } 22^{\circ} 03^{\prime}\end{array}$ & ootheca & $\begin{array}{l}\text { ecotone; forest } \\
\text { edge/fallow lands }\end{array}$ & $\begin{array}{l}\text { dry sandy grassland } \\
\text { Corynophoretum }\end{array}$ & $\uparrow$ \\
\hline 56 & $\begin{array}{l}\text { Korytków Duży near } \\
\text { Bilgoraj }\end{array}$ & FB10 & $\begin{array}{l}\text { N } 50^{\circ} 37^{\prime} \\
\text { E } 22^{\circ} 40^{\prime}\end{array}$ & ootheca & $\begin{array}{l}\text { ecotone; forest } \\
\text { edge }\end{array}$ & $\begin{array}{l}\text { dry sandy grassland } \\
\text { Corynophoretum }\end{array}$ & $\uparrow$ \\
\hline 57 & $\begin{array}{l}\text { Kosowy for. } 262 \text { near } \\
\text { Mielec }\end{array}$ & EA47 & $\begin{array}{l}\text { N } 50^{\circ} 17^{\prime} \\
\text { E } 21^{\circ} 36^{\prime}\end{array}$ & $\begin{array}{l}\text { juv., } \partial, \rho, \\
\text { ootheca }\end{array}$ & $\begin{array}{l}\text { zone deforested by } \\
\text { transmission line }\end{array}$ & $\begin{array}{l}\text { Arctostaphylo- } \\
\text { Callunetum in a pinewood }\end{array}$ & $\stackrel{\infty}{\leftrightarrow}$ \\
\hline 58 & $\begin{array}{l}\text { Kotowa Wola I near } \\
\text { Tarnobrzeg }\end{array}$ & EB60 & $\begin{array}{l}\text { N } 50^{\circ} 37^{\prime} \\
\text { E } 21^{\circ} 57^{\prime}\end{array}$ & ootheca & $\begin{array}{l}\text { ecotone; forest } \\
\text { margin/fallow }\end{array}$ & $\begin{array}{l}\text { dry sandy grassland } \\
\text { Corynophoretum }\end{array}$ & $\uparrow$ \\
\hline 59 & $\begin{array}{l}\text { Kotowa Wola II near } \\
\text { Tarnobrzeg }\end{array}$ & EB60 & $\begin{array}{l}\text { N } 50^{\circ} 37^{\prime} \\
\text { E } 21^{\circ} 56^{\prime}\end{array}$ & ootheca & $\begin{array}{l}\text { westland near } \\
\text { forest margin }\end{array}$ & $\begin{array}{l}\text { dry sandy grassland } \\
\text { Corynophoretum }\end{array}$ & $\uparrow$ \\
\hline 60 & $\begin{array}{l}\text { Kowale Podlesie near } \\
\text { Sokołów Młp. }\end{array}$ & EA77 & $\begin{array}{l}\text { N } 50^{\circ} 19^{\prime} \\
\text { E } 22^{\circ} 05^{\prime}\end{array}$ & ootheca & $\begin{array}{l}\text { ecotone; forest } \\
\text { edge/fallow }\end{array}$ & $\begin{array}{l}\text { dry sandy grassland } \\
\text { Corynophoretum }\end{array}$ & $\uparrow$ \\
\hline 61 & $\begin{array}{l}\text { Krasne Wólka } \\
\text { Niedźwiedzka }\end{array}$ & EA87 & $\begin{array}{l}\text { N } 50^{\circ} 16^{\prime} \\
\text { E } 22^{\circ} 14^{\prime}\end{array}$ & ootheca & $\begin{array}{l}\text { forest edge/ } \\
\text { meadow drained }\end{array}$ & $\begin{array}{l}\text { dry sandy grassland } \\
\text { Corynophoretum }\end{array}$ & $\uparrow$ \\
\hline 62 & $\begin{array}{l}\text { Krzątka near Nowa } \\
\text { Dęba }\end{array}$ & EA58 & $\begin{array}{l}\text { N } 50^{\circ} 25^{\prime} \\
\text { E } 21^{\circ} 49^{\prime}\end{array}$ & $\begin{array}{l}.8, \text {, } \\
\text { ootheca }\end{array}$ & $\begin{array}{l}\text { forest edge/ } \\
\text { fallow/meadow }\end{array}$ & $\begin{array}{l}\text { heath/dry sandy } \\
\text { grassland/ meadow }\end{array}$ & $\stackrel{\infty}{\leftrightarrow}$ \\
\hline 63 & $\begin{array}{l}\text { Kurylówka near } \\
\text { Leżajsk }\end{array}$ & FA07 & $\begin{array}{l}\text { N } 50^{\circ} 19^{\prime} \\
\text { E } 22^{\circ} 28^{\prime}\end{array}$ & 9 , ootheca & open; fellow lands & $\begin{array}{l}\text { dry sandy } \\
\text { grassland/ruderal }\end{array}$ & $\uparrow$ \\
\hline 64 & Kusze near Harasiuki & FA09 & $\begin{array}{l}\text { N } 50^{\circ} 28^{\prime} \\
\text { E } 22^{\circ} 26^{\prime}\end{array}$ & q, ootheca & $\begin{array}{l}\text { ecotone; forest } \\
\text { edge/fallow }\end{array}$ & $\begin{array}{l}\text { dry sandy grassland } \\
\text { Corynophoretum }\end{array}$ & $\leftrightarrow ?$ \\
\hline 65 & $\begin{array}{l}\text { Leszcze I near } \\
\text { Kolbuszowa }\end{array}$ & EA45 & $\begin{array}{l}\text { N } 50^{\circ} 10^{\prime} \\
\text { E } 21^{\circ} 39^{\prime}\end{array}$ & $\begin{array}{l}\nexists, q \\
\text { ootheca }\end{array}$ & $\begin{array}{l}\text { ecotone; forest } \\
\text { edge/fallow }\end{array}$ & $\begin{array}{l}\text { dry sandy grassland } \\
\text { Corynophoretum }\end{array}$ & $\uparrow$ \\
\hline
\end{tabular}

\footnotetext{
${ }^{5}$ Based on a specimen captured by schoolboy in Kolbuszowa, and information of F. Cepielik and a field inspection by author
} 


\begin{tabular}{|c|c|c|c|c|c|c|c|}
\hline 1 & 2 & 3 & 4 & 5 & 6 & 7 & 8 \\
\hline 66 & $\begin{array}{l}\text { Leszcze II near } \\
\text { Kolbuszowa }\end{array}$ & EA46 & $\begin{array}{l}\text { N } 50^{\circ} 11^{\prime} \\
\text { E } 21^{\circ} 39^{\prime}\end{array}$ & $\begin{array}{l}\text { juv., d, }, \text {, } \\
\text { ootheca }\end{array}$ & $\begin{array}{l}\text { ecotone; forest } \\
\text { edge/fallow }\end{array}$ & $\begin{array}{l}\text { dry sandy grassland } \\
\text { Corynophoretum }\end{array}$ & $\uparrow$ \\
\hline 67 & $\begin{array}{l}\text { Leszcze for. } 243 \text { / } \\
244 / 290 .\end{array}$ & EA46 & $\begin{array}{l}\text { N } 50^{\circ} 11^{\prime} \\
\text { E } 21^{\circ} 37^{\prime}\end{array}$ & O, ootheca & $\begin{array}{l}\text { ecotone; forest } \\
\text { track }\end{array}$ & $\begin{array}{l}\text { heath and pinewood } \\
\text { undergrowth }\end{array}$ & $\stackrel{\infty}{\leftrightarrow}$ \\
\hline 68 & $\begin{array}{l}\text { Lipnica near } \\
\text { Kolbuszowa }\end{array}$ & EA67 & $\begin{array}{l}\text { N } 50^{\circ} 19^{\prime} \\
\text { E } 21^{\circ} 55^{\prime}\end{array}$ & $\begin{array}{l}\delta, 9 \\
\text { ootheca }\end{array}$ & $\begin{array}{l}\text { ecotone; forest } \\
\text { edge/fallow }\end{array}$ & $\begin{array}{l}\text { heath/dry sandy } \\
\text { grassland }\end{array}$ & $\leftrightarrow$ \\
\hline 69 & Ląkieć - Wilcza Wola & EA67 & $\begin{array}{l}\text { N } 50^{\circ} 21^{\prime} \\
\text { E } 21^{\circ} 59^{\prime}\end{array}$ & ootheca & $\begin{array}{l}\text { ecotone; forest } \\
\text { edge/fallow }\end{array}$ & $\begin{array}{l}\text { dry sandy grassland } \\
\text { Corynophoretum }\end{array}$ & $\leftrightarrow$ \\
\hline 70 & $\begin{array}{l}\text { Lą̇̇ek Ordynacki near } \\
\text { Janów Lub. }\end{array}$ & EB90 & $\begin{array}{l}\text { N } 50^{\circ} 37^{\prime} \\
\text { E } 22^{\circ} 17\end{array}$ & ootheca & $\begin{array}{l}\text { ecotone; forest } \\
\text { edge/fallow }\end{array}$ & $\begin{array}{l}\text { dry sandy grassland } \\
\text { Corynophoretum }\end{array}$ & $\overleftrightarrow{\uparrow}$ \\
\hline 71 & Lętownia near Rudnik & EA87 & $\begin{array}{l}\text { N } 50^{\circ} 20^{\prime} \\
\text { E } 22^{\circ} 12^{\prime}\end{array}$ & ootheca & open; sand-pit & $\begin{array}{l}\text { dry sandy grassland initial } \\
\text { /ruderal }\end{array}$ & $\uparrow$ \\
\hline 72 & $\begin{array}{l}\text { Lętownia Babiarze } \\
\text { near Rudnik }\end{array}$ & $\mathrm{Ea} 87$ & $\begin{array}{l}\text { N } 50^{\circ} 21^{\prime} \\
\text { E } 22^{\circ} 14^{\prime}\end{array}$ & ootheca & $\begin{array}{l}\text { ecotone; forest } \\
\text { edge/fallow }\end{array}$ & $\begin{array}{l}\text { dry sandy } \\
\text { grassland/segetal }\end{array}$ & $\uparrow$ \\
\hline 73 & $\begin{array}{l}\text { Lugnica Kolonia near } \\
\text { Mielec }\end{array}$ & EA47 & $\begin{array}{l}\text { N } 50^{\circ} 18^{\prime} \\
\text { E } 21^{\circ} 36^{\prime}\end{array}$ & ootheca & $\begin{array}{l}\text { ecotone; large } \\
\text { clearing }\end{array}$ & $\begin{array}{l}\text { dry sandy grassland } \\
\text { Corynophoretum }\end{array}$ & $\leftrightarrow$ \\
\hline 74 & Luże I near Mielec & EA46 & $\begin{array}{l}\text { N } 50^{\circ} 17^{\prime} \\
\text { E } 21^{\circ} 33^{\prime}\end{array}$ & ootheca & $\begin{array}{l}\text { ecotone ; small } \\
\text { clearings }\end{array}$ & $\begin{array}{l}\text { pine wood undergrowth/ } \\
\text { dry sandy grassland }\end{array}$ & $\leftrightarrow$ \\
\hline 75 & Luże II near Mielec & EA46 & $\begin{array}{l}\text { N } 50^{\circ} 16^{\prime} \\
\text { E } 21^{\circ} 34^{\prime}\end{array}$ & 9, ootheca & $\begin{array}{l}\text { ecotone; large } \\
\text { clearing }\end{array}$ & $\begin{array}{l}\text { dry sandy grassland } \\
\text { Corynophoretum }\end{array}$ & $\leftrightarrow$ \\
\hline 76 & $\begin{array}{l}\text { Lysaków Kolonia near } \\
\text { Zaklików }\end{array}$ & EB82 & $\begin{array}{l}\text { N } 50^{\circ} 45^{\prime} \\
\text { E } 22^{\circ} 09^{\prime}\end{array}$ & O, ootheca & $\begin{array}{l}\text { ecotone; large } \\
\text { clearing }\end{array}$ & $\begin{array}{l}\text { heath/dry sandy } \\
\text { grassland }\end{array}$ & $\stackrel{\infty}{\leftrightarrow}$ \\
\hline 77 & $\begin{array}{l}\text { Majdan Królewski } \\
\text { near Nowa Dęba }\end{array}$ & EA58 & $\begin{array}{l}\text { N } 50^{\circ} 21^{\prime} \\
\text { E } 21^{\circ} 43^{\prime}\end{array}$ & $\begin{array}{l}\text { juv., } \partial, q, \\
\text { ootheca }\end{array}$ & $\begin{array}{l}\text { ecotone; large } \\
\text { clearing }\end{array}$ & $\begin{array}{l}\text { dry sandy grassland } \\
\text { Corynophoretum }\end{array}$ & $\stackrel{\infty}{\leftrightarrow}$ \\
\hline 78 & $\begin{array}{l}\text { Marynin - Wólka } \\
\text { Niedźwiedzka }\end{array}$ & EA 87 & $\begin{array}{l}\text { N } 50^{\circ} 16^{\prime} \\
\text { E } 22^{\circ} 13^{\prime}\end{array}$ & ootheca & $\begin{array}{l}\text { ecotone; forest } \\
\text { edge/fallow }\end{array}$ & $\begin{array}{l}\text { dry sandy } \\
\text { grassland/segetal }\end{array}$ & $\uparrow$ \\
\hline 79 & $\begin{array}{l}\text { Mechowiec near } \\
\text { Kolbuszowa }\end{array}$ & EA57 & $\begin{array}{l}\text { N } 50^{\circ} 18^{\prime} \\
\text { E } 21^{\circ} 48^{\prime}\end{array}$ & O, ootheca & $\begin{array}{l}\text { ecotone; forest } \\
\text { edge/fallow }\end{array}$ & $\begin{array}{l}\text { meadow/dry sandy } \\
\text { grassland }\end{array}$ & $\leftrightarrow$ \\
\hline 80 & $\begin{array}{l}\text { Moskale I- Nisko } \\
\text { near Stalowa Wola }\end{array}$ & EA79 & $\begin{array}{l}\text { N } 50^{\circ} 32^{\prime} \\
\text { E } 22^{\circ} 06^{\prime}\end{array}$ & $\delta, 9$ & $\begin{array}{l}\text { ecotone; forest } \\
\text { edge/fallow }\end{array}$ & $\begin{array}{l}\text { meadow/dry sandy } \\
\text { grassland }\end{array}$ & $\leftrightarrow$ \\
\hline 81 & $\begin{array}{l}\text { Moskale II - for. } \\
327 / 342\end{array}$ & EA79 & $\begin{array}{l}\text { N } 50^{\circ} 32^{\prime} \\
\text { E } 22^{\circ} 05^{\prime}\end{array}$ & 9 & $\begin{array}{l}\text { ecotone; small } \\
\text { clearing }\end{array}$ & $\begin{array}{l}\text { heath/dry sandy } \\
\text { grassland in pinewood }\end{array}$ & $\stackrel{\infty}{\leftrightarrow}$ \\
\hline 82 & $\begin{array}{l}\text { Nadrzecze near } \\
\text { Bilgoraj }\end{array}$ & FB10 & $\begin{array}{l}\text { N } 50^{\circ} 36^{\prime} \\
\text { E } 22^{\circ} 40^{\prime}\end{array}$ & ootheca & $\begin{array}{l}\text { ecotone; small } \\
\text { clearing }\end{array}$ & $\begin{array}{l}\text { dry sandy grassland } \\
\text { Corynophoretum }\end{array}$ & $\uparrow$ \\
\hline 83 & Nisko - for. 19 Barce & EA89 & $\begin{array}{l}\text { N } 50^{\circ} 31^{\prime} \\
\text { E } 22^{\circ} 06^{\prime}\end{array}$ & 9 , ootheca & $\begin{array}{l}\text { ecotone; small } \\
\text { clearing }\end{array}$ & $\begin{array}{l}\text { heath/dry sandy } \\
\text { grassland }\end{array}$ & $\stackrel{\infty}{\leftrightarrow}$ \\
\hline 84 & $\begin{array}{l}\text { Niwiska I } \\
\text { near Kolbuszowa }\end{array}$ & EA46 & $\begin{array}{l}\text { N } 50^{\circ} 12^{\prime} \\
\text { E } 21^{\circ} 38^{\prime}\end{array}$ & 9, ootheca & $\begin{array}{l}\text { ecotone; small } \\
\text { clearing }\end{array}$ & $\begin{array}{l}\text { dry sandy grassland } \\
\text { Corynophoretum }\end{array}$ & $\leftrightarrow$ \\
\hline 85 & $\begin{array}{l}\text { Niwiska II } \\
\text { near Kolbuszowa }\end{array}$ & EA46 & $\begin{array}{l}\text { N } 50^{\circ} 12^{\prime} \\
\text { E } 21^{\circ} 37^{\prime}\end{array}$ & $\begin{array}{l}\text { juv., ठ, }, q, \\
\text { ootheca }\end{array}$ & $\begin{array}{l}\text { ecotone; large } \\
\text { clearing, fallows }\end{array}$ & $\begin{array}{l}\text { dry sandy grassland } \\
\text { /segetal/meadow }\end{array}$ & $\leftrightarrow$ \\
\hline 86 & $\begin{array}{l}\text { Niwiska III } \\
\text { near Kolbuszowa }\end{array}$ & EA46 & $\begin{array}{l}\text { N } 50^{\circ} 12^{\prime} \\
\text { E } 21^{\circ} 37^{\prime}\end{array}$ & q, ootheca & $\begin{array}{l}\text { ecotone; large } \\
\text { clearing, fallows }\end{array}$ & $\begin{array}{l}\text { dry sandy grassland/ } \\
\text { segetal/meadow }\end{array}$ & $\leftrightarrow$ \\
\hline 87 & $\begin{array}{l}\text { Niwiska IV } \\
\text { near Kolbuszowa }\end{array}$ & EA46 & $\begin{array}{l}\text { N } 50^{\circ} 13^{\prime} \\
\text { E } 21^{\circ} 36\end{array}$ & q, ootheca & $\begin{array}{l}\text { ecotone; forest } \\
\text { edge/fallow }\end{array}$ & $\begin{array}{l}\text { dry sandy grassland } \\
\text { Corynophoretum }\end{array}$ & $\leftrightarrow$ \\
\hline 88 & $\begin{array}{l}\text { Nowa Dęba, for. } 306 \\
\text { Rozalin }\end{array}$ & EA58 & $\begin{array}{l}\text { N } 50^{\circ} 26^{\prime} \\
\text { E } 21^{\circ} 43^{\prime}\end{array}$ & $\delta$ & $\begin{array}{l}\text { ecotone; small } \\
\text { clearing }\end{array}$ & $\begin{array}{l}\text { pinewood undergrowth/ } \\
\text { dry sandy grassland }\end{array}$ & $\stackrel{\infty}{\leftrightarrow}$ \\
\hline 89 & $\begin{array}{l}\text { Obojna near Stalowa } \\
\text { Wola }\end{array}$ & EB70 & $\begin{array}{l}\text { N } 50^{\circ} 37^{\prime} \\
\text { E } 21^{\circ} 59^{\prime}\end{array}$ & ootheca & $\begin{array}{l}\text { open; fallow } \\
\text { lands, sand-pit }\end{array}$ & $\begin{array}{l}\text { dry sandy grassland } \\
\text { Corynophoretum }\end{array}$ & $\leftrightarrow$ \\
\hline 90 & Ocice near Tarnobrzeg & EB40 & $\begin{array}{l}\text { N } 50^{\circ} 33^{\prime} \\
\text { E } 21^{\circ} 40^{\prime}\end{array}$ & 9, ootheca & $\begin{array}{l}\text { forest edge/ } \\
\text { fallows/meadow }\end{array}$ & $\begin{array}{l}\text { heath/dry sandy } \\
\text { grassland }\end{array}$ & $\leftrightarrow$ \\
\hline 91 & $\begin{array}{l}\text { Ocieka near Sędziszów } \\
\text { Malopolski }\end{array}$ & EA45 & $\begin{array}{l}\text { N } 50^{\circ} 09^{\prime} \\
\text { E } 21^{\circ} 35^{\prime}\end{array}$ & $\begin{array}{l}\text { juv., d, } q, \\
\text { ootheca }\end{array}$ & $\begin{array}{l}\text { ecotone; forest } \\
\text { edge/fallow }\end{array}$ & $\begin{array}{l}\text { dry sandy grassland } \\
\text { Corynophoretum }\end{array}$ & $\leftrightarrow$ \\
\hline 92 & $\begin{array}{l}\text { Ostrowy Baranowskie } \\
\text { Pateraki }\end{array}$ & EA47 & $\begin{array}{l}\text { N } 50^{\circ} 20^{\prime} \\
\text { E } 21^{\circ} 38^{\prime}\end{array}$ & q, ootheca & $\begin{array}{l}\text { ecotone; forest } \\
\text { edge/fallow }\end{array}$ & $\begin{array}{l}\text { dry sandy grassland } \\
\text { Corynophoretum }\end{array}$ & $\uparrow$ \\
\hline 93 & $\begin{array}{l}\text { Ostrowy Baranowskie } \\
\text { Poręba }\end{array}$ & EA48 & $\begin{array}{l}\text { N } 50^{\circ} 21^{\prime} \\
\text { E } 21^{\circ} 37^{\prime}\end{array}$ & ootheca & $\begin{array}{l}\text { ecotone; forest } \\
\text { edge/fallow }\end{array}$ & $\begin{array}{l}\text { dry sandy grassland } \\
\text { Corynophoretum }\end{array}$ & $\leftrightarrow$ \\
\hline 94 & $\begin{array}{l}\text { Pikuły - Jeżowe near } \\
\text { Rudnik }\end{array}$ & EA88 & $\begin{array}{l}\text { N } 50^{\circ} 23^{\prime} \\
\text { E } 22^{\circ} 12^{\prime}\end{array}$ & q, ootheca & $\begin{array}{l}\text { ecotone; forest } \\
\text { edge/fallow }\end{array}$ & $\begin{array}{l}\text { heath/dry sandy } \\
\text { grassland }\end{array}$ & $\uparrow$ \\
\hline
\end{tabular}




\begin{tabular}{|c|c|c|c|c|c|c|c|}
\hline 1 & 2 & 3 & 4 & 5 & 6 & 7 & 8 \\
\hline 95 & $\begin{array}{l}\text { Płazówka near } \\
\text { Kolbuszowa }\end{array}$ & EA67 & $\begin{array}{l}\text { N } 50^{\circ} 19^{\prime} \\
\text { E } 21^{\circ} 50^{\prime}\end{array}$ & ootheca & $\begin{array}{l}\text { ecotone; forest } \\
\text { edge/fallow }\end{array}$ & $\begin{array}{l}\text { heath/dry sandy } \\
\text { grassland }\end{array}$ & $\leftrightarrow$ \\
\hline 96 & $\begin{array}{l}\text { Pniaki Wilcza Wola } \\
\text { near Kolbuszowa }\end{array}$ & EA78 & $\begin{array}{l}\text { N } 50^{\circ} 21^{\prime} \\
\text { E } 21^{\circ} 59^{\prime}\end{array}$ & $\begin{array}{l}\text { juv., } d, q, \\
\text { ootheca }\end{array}$ & open; meadow & $\begin{array}{l}\text { meadow with Juncus } \\
\text { effusus drained }\end{array}$ & $\leftrightarrow$ \\
\hline 97 & $\begin{array}{l}\text { Podłężniówka near } \\
\text { Kolbuszowa }\end{array}$ & EA57 & $\begin{array}{l}\text { N } 50^{\circ} 18^{\prime} \\
\text { E } 21^{\circ} 49^{\prime}\end{array}$ & ootheca & $\begin{array}{l}\text { ecotone; forest } \\
\text { edge/fallow }\end{array}$ & heath/dry sandy grassland & $\uparrow$ \\
\hline 98 & Podole near Przecław & EA36 & $\begin{array}{l}\mathrm{N} 50^{\circ} 11^{\prime} \\
\mathrm{E} 21^{\circ} 27^{\prime}\end{array}$ & 9, ootheca & $\begin{array}{l}\text { ecotone; forest } \\
\text { edge/fallow }\end{array}$ & $\begin{array}{l}\text { dry sandy grassland/ } \\
\text { meadow }\end{array}$ & $\uparrow$ \\
\hline 99 & Podwolina near Nisko & EA 89 & $\begin{array}{l}\text { N } 50^{\circ} 30^{\prime} \\
\text { E } 22^{\circ} 10^{\prime}\end{array}$ & q, ootheca & $\begin{array}{l}\text { ecotone; forest } \\
\text { edge/fallow }\end{array}$ & $\begin{array}{l}\text { dry sandy grassland } \\
\text { Corynophoretum }\end{array}$ & $\leftrightarrow$ \\
\hline 100 & $\begin{array}{l}\text { Pogwizdów near } \\
\text { Raniżów }\end{array}$ & EA 66 & $\begin{array}{l}\text { N } 50^{\circ} 15^{\prime} \\
\text { E } 21^{\circ} 56^{\prime}\end{array}$ & $\begin{array}{l}3, q \\
\text { ootheca }\end{array}$ & $\begin{array}{l}\text { ecotone; forest } \\
\text { edge/meadows }\end{array}$ & $\begin{array}{l}\text { meadow with Nardus } \\
\text { stricta }\end{array}$ & $\leftrightarrow$ \\
\hline 101 & $\begin{array}{l}\text { Poręby Huciskie near } \\
\text { Kolbuszowa }\end{array}$ & EA46 & $\begin{array}{l}\text { N } 50^{\circ} 11^{\prime} \\
\text { E } 21^{\circ} 40^{\prime}\end{array}$ & $\begin{array}{l}\text { juv., }, \text {, } \\
\text { ootheca }\end{array}$ & open; fallow lands & $\begin{array}{l}\text { dry sandy grassland } \\
\text { Corynophoretum }\end{array}$ & intro? \\
\hline 102 & $\begin{array}{l}\text { Potok Górny near } \\
\text { Tarnogród }\end{array}$ & FA18 & $\begin{array}{l}\text { N } 50^{\circ} 25^{\prime} \\
\text { E } 22^{\circ} 32^{\prime}\end{array}$ & 우 & $\begin{array}{l}\text { ecotone; forest } \\
\text { edge/meadows }\end{array}$ & $\begin{array}{l}\text { wet meadow with } \\
\text { Gentiana pneumonanthe }\end{array}$ & $\uparrow$ \\
\hline 103 & Przędzel near Rudnik & EA89 & $\begin{array}{l}\text { N } 50^{\circ} 29^{\prime} \\
\text { E } 22^{\circ} 10^{\prime}\end{array}$ & ootheca & $\begin{array}{l}\text { ecotone; forest } \\
\text { edge/fallow }\end{array}$ & $\begin{array}{l}\text { dry sandy grassland/ } \\
\text { ruderal }\end{array}$ & $\leftrightarrow$ \\
\hline 104 & $\begin{array}{l}\text { Przylęk Marmury near } \\
\text { Mielec }\end{array}$ & EA47 & $\begin{array}{l}\text { N } 50^{\circ} 17^{\prime} \\
\text { E } 21^{\circ} 36\end{array}$ & $\begin{array}{l}\text { juv., } \delta, q, \\
\text { ootheca }\end{array}$ & $\begin{array}{l}\text { ecotone; forest } \\
\text { edge/fallow }\end{array}$ & $\begin{array}{l}\text { dry sandy grassland } \\
\text { Corynophoretum }\end{array}$ & $\leftrightarrow$ \\
\hline 105 & $\begin{array}{l}\text { Przylęk for. near } \\
\text { Mielec. }\end{array}$ & EA47 & $\begin{array}{l}\text { N } 50^{\circ} 17^{\prime} \\
\text { E } 21^{\circ} 35\end{array}$ & $\begin{array}{l}3,9 \\
\text { ootheca }\end{array}$ & $\begin{array}{l}\text { ecotone: clearing } \\
\text { in pinewood }\end{array}$ & heath/dry sandy grassland & $\stackrel{\infty}{\leftrightarrow}$ \\
\hline 106 & $\begin{array}{l}\text { Radomyśl artillery } \\
\text { range }\end{array}$ & EB62 & $\begin{array}{l}\text { N } 50^{\circ} 43 \\
\text { E } 21^{\circ} 58^{\prime}\end{array}$ & $\begin{array}{l}\text { imago, } \\
\text { ootheca }\end{array}$ & $\begin{array}{l}\text { open; military } \\
\text { training ground }\end{array}$ & heath/dry sandy grassland & $\infty$ \\
\hline 107 & Rudnik Doliny & EA88 & $\begin{array}{l}\text { N } 50^{\circ} 25^{\prime} \\
\text { E } 22^{\circ} 13\end{array}$ & $\begin{array}{l}\text { juv., } \hat{\delta}, q, \\
\text { ootheca }\end{array}$ & $\begin{array}{l}\text { ecotone; forest } \\
\text { edge/fallow }\end{array}$ & $\begin{array}{l}\text { dry sandy grassland } \\
\text { Corynophoretum }\end{array}$ & $\stackrel{\infty}{\leftrightarrow}$ \\
\hline 108 & $\begin{array}{l}\text { Rudnik near Forest } \\
\text { District Office }\end{array}$ & EA 88 & $\begin{array}{l}\text { N } 50^{\circ} 25^{\prime} \\
\text { E } 22^{\circ} 14\end{array}$ & 9, ootheca & $\begin{array}{l}\text { ecotone; forest } \\
\text { edge/fallow }\end{array}$ & $\begin{array}{l}\text { dry sandy } \\
\text { grassland/ruderal }\end{array}$ & $\leftrightarrow$ \\
\hline 109 & $\begin{array}{l}\text { Rudnik station on the } \\
\text { San river }\end{array}$ & EA 89 & $\begin{array}{l}\text { N } 50^{\circ} 26^{\prime} \\
\text { E } 22^{\circ} 15^{\prime}\end{array}$ & q & $\begin{array}{l}\text { ecotone; forest } \\
\text { edge/fallow }\end{array}$ & $\begin{array}{l}\text { dry sandy } \\
\text { grassland/ruderal }\end{array}$ & $\leftrightarrow$ \\
\hline 110 & $\begin{array}{l}\text { Rusinów near Nowa } \\
\text { Dęba }\end{array}$ & EA58 & $\begin{array}{l}\text { N } 50^{\circ} 22^{\prime} \\
\mathrm{E} 21^{\circ} 48^{\prime}\end{array}$ & ootheca & $\begin{array}{l}\text { ecotone; forest } \\
\text { edge/fallow }\end{array}$ & $\begin{array}{l}\text { dry sandy grassland } \\
\text { Corynophoretum scarce }\end{array}$ & $\uparrow$ \\
\hline 111 & Sarnów I near Mielec & EA38 & $\begin{array}{l}\text { N } 50^{\circ} 21^{\prime} \\
\text { E } 21^{\circ} 32^{\prime}\end{array}$ & q, ootheca & wastlands & $\begin{array}{l}\text { dry sandy } \\
\text { grassland/rudera }\end{array}$ & $\leftrightarrow$ \\
\hline 112 & Sarnów II near Mielec & EA38 & $\begin{array}{l}\text { N } 50^{\circ} 22^{\prime} \\
\text { E } 21^{\circ} 31^{\prime}\end{array}$ & $\begin{array}{l}\delta, q \\
\text { ootheca }\end{array}$ & open fallow lands & $\begin{array}{l}\text { dry sandy grassland } \\
\text { Corynophoretum }\end{array}$ & $\leftrightarrow$ \\
\hline 113 & $\begin{array}{l}\text { Sieraków near } \\
\text { Harasiuki }\end{array}$ & FA09 & $\begin{array}{l}\text { N } 50^{\circ} 28^{\prime} \\
\text { E } 22^{\circ} 25^{\prime}\end{array}$ & ootheca & $\begin{array}{l}\text { ecotone; forest } \\
\text { edge/fallow }\end{array}$ & $\begin{array}{l}\text { dry sandy grassland } \\
\text { Corynophoretum }\end{array}$ & $\leftrightarrow$ \\
\hline 114 & $\begin{array}{l}\text { Sieraków II near } \\
\text { Harasiuki }\end{array}$ & FA09 & $\begin{array}{l}\text { N } 50^{\circ} 28^{\prime} \\
\text { E } 22^{\circ} 25^{\prime}\end{array}$ & 9, ootheca & $\begin{array}{l}\text { ecotone; small } \\
\text { clearing }\end{array}$ & $\begin{array}{l}\text { dry sandy grassland } \\
\text { Corynophoretum }\end{array}$ & $\leftrightarrow$ \\
\hline 115 & $\begin{array}{l}\text { Sobów near } \\
\text { Tarnobrzeg }\end{array}$ & EB50 & $\begin{array}{l}\text { N } 50^{\circ} 37^{\prime} \\
\text { E } 21^{\circ} 45^{\prime}\end{array}$ & ootheca & $\begin{array}{l}\text { ecotone; forest } \\
\text { edge/fallow }\end{array}$ & $\begin{array}{l}\text { dry sandy grassland } \\
\text { Corynophoretum }\end{array}$ & $\uparrow$ \\
\hline 116 & Soputek for. Barce & EA79 & $\begin{array}{l}\text { N } 50^{\circ} 28^{\prime} \\
\text { E } 22^{\circ} 06^{\prime}\end{array}$ & 9, ootheca & $\begin{array}{l}\text { ecotone; clearing } \\
\text { - wood meadow }\end{array}$ & sedge meadow drained & $\leftrightarrow$ \\
\hline 117 & Sól near Bitgoraj & FA19 & $\begin{array}{l}\text { N } 50^{\circ} 29^{\prime} \\
\text { E } 22^{\circ} 37^{\prime}\end{array}$ & ootheca & $\begin{array}{l}\text { ecotone; forest } \\
\text { edge/fallow }\end{array}$ & $\begin{array}{l}\text { dry sandy } \\
\text { grassland/meadow }\end{array}$ & $\uparrow$ \\
\hline 118 & Stalowa Wola Chyły & EB70 & $\begin{array}{l}\text { N } 50^{\circ} 32^{\prime} \\
\text { E } 22^{\circ} 04^{\prime}\end{array}$ & $\delta$ & building ad lucem & $\begin{array}{l}\text { sandy fallow lands in } \\
\text { vicinity }\end{array}$ & $\downarrow$ \\
\hline 119 & Stany near Przyszów & EA 69 & $\begin{array}{l}\text { N } 50^{\circ} 27^{\prime} \\
\text { E } 21^{\circ} 57^{\prime}\end{array}$ & ootheca & $\begin{array}{l}\text { ecotone; forest } \\
\text { edge/fallow }\end{array}$ & $\begin{array}{l}\text { dry sandy grassland } \\
\text { Corynophoretum }\end{array}$ & $\leftrightarrow$ \\
\hline 120 & Stróża I near Rudnik & EA89 & $\begin{array}{l}\text { N } 50^{\circ} 28^{\prime} \\
\text { E } 22^{\circ} 13^{\prime}\end{array}$ & Q, ootheca & $\begin{array}{l}\text { ecotone; large } \\
\text { clearing }\end{array}$ & $\begin{array}{l}\text { dry sandy grassland } \\
\text { Corynophoretum }\end{array}$ & $\leftrightarrow$ \\
\hline 121 & Stróża II near Rudnik & EA89 & $\begin{array}{l}\text { N } 50^{\circ} 27^{\prime} \\
\text { E } 22^{\circ} 12^{\prime}\end{array}$ & $\begin{array}{l}\delta, q \\
\text { ootheca }\end{array}$ & $\begin{array}{l}\text { ecotone; forest } \\
\text { edge/fallow }\end{array}$ & heath/dry sandy grassland & $\stackrel{\infty}{\leftrightarrow}$ \\
\hline 122 & $\begin{array}{l}\text { Szydłowieckie Ląi } \\
\text { near Mielec }\end{array}$ & EA47 & $\begin{array}{l}\text { N } 50^{\circ} 20^{\prime} \\
\text { E } 21^{\circ} 32^{\prime}\end{array}$ & ootheca & ecotone; clearing & $\begin{array}{l}\text { wet meadow with Juncus } \\
\text { effusus drained }\end{array}$ & $\leftrightarrow$ \\
\hline 123 & $\begin{array}{l}\text { Tarnowska Wola near } \\
\text { Tarnobrzeg }\end{array}$ & EA58 & $\begin{array}{l}\text { N } 50^{\circ} 28^{\prime} \\
\text { E } 21^{\circ} 42^{\prime}\end{array}$ & ootheca & $\begin{array}{l}\text { ecotone; forest } \\
\text { edge/meadow }\end{array}$ & $\begin{array}{l}\text { wet meadow with Juncus } \\
\text { effusus drained }\end{array}$ & $\leftrightarrow$ \\
\hline
\end{tabular}


A. Liana

\begin{tabular}{|c|c|c|c|c|c|c|c|}
\hline 1 & 2 & 3 & 4 & 5 & 6 & 7 & 8 \\
\hline 124 & $\begin{array}{l}\text { Trzosowa Ścieżka near } \\
\text { Nowa Dęba }\end{array}$ & EA68 & \begin{tabular}{|l|} 
N $50^{\circ} 23^{\prime}$ \\
E $21^{\circ} 52^{\prime}$
\end{tabular} & ootheca & open; fallow lands & dry sandy/meadow & $\leftrightarrow$ \\
\hline 125 & $\begin{array}{l}\text { Wilcza Góra - Nowa } \\
\text { Dęba }\end{array}$ & EA59 & $\begin{array}{l}\text { N } 50^{\circ} 27^{\prime} \\
\text { E } 21^{\circ} 48^{\prime}\end{array}$ & Imago & $\begin{array}{l}\text { open; military } \\
\text { training ground }\end{array}$ & heath/dry sandy grassland & $\infty$ \\
\hline 126 & Wilcza Wola Szwedy & EA67 & $\begin{array}{l}\text { N } 50^{\circ} 20^{\prime} \\
\text { E } 21^{\circ} 57^{\prime}\end{array}$ & 9 , ootheca & $\begin{array}{l}\text { ecotone; forest } \\
\text { edge/fallow }\end{array}$ & heath/dry sandy grassland & $\leftrightarrow$ \\
\hline 127 & Wilcza Wola Zapole & EA68 & $\begin{array}{l}\text { N } 50^{\circ} 20^{\prime} \\
\text { E } 21^{\circ} 53^{\prime}\end{array}$ & ootheca & $\begin{array}{l}\text { ecotone; forest } \\
\text { edge/fallow }\end{array}$ & $\begin{array}{l}\text { dry sandy grassland with } \\
\text { young pine growth }\end{array}$ & $\leftrightarrow$ \\
\hline 128 & Wilcza Wola Zmysłów & EA67 & $\begin{array}{l}\text { N } 50^{\circ} 20^{\prime} \\
\text { E } 21^{\circ} 54^{\prime}\end{array}$ & $\begin{array}{l}\text { juv., } \delta, q, \\
\text { ootheca }\end{array}$ & $\begin{array}{l}\text { ecotone; large } \\
\text { clearing }\end{array}$ & $\begin{array}{l}\text { heath/ dry sandy } \\
\text { grassland }\end{array}$ & $\begin{array}{l}\infty \\
\leftrightarrow\end{array}$ \\
\hline 129 & $\begin{array}{l}\text { Wólka Lamana near } \\
\text { Kulno }\end{array}$ & FA07 & $\begin{array}{l}\text { N } 50^{\circ} 20^{\prime} \\
\text { E } 22^{\circ} 31^{\prime}\end{array}$ & ootheca & $\begin{array}{l}\text { ecotone; forest } \\
\text { edge/fallow }\end{array}$ & $\begin{array}{l}\text { dry sandy grassland } \\
\text { Corynophoretum }\end{array}$ & $\uparrow$ \\
\hline 130 & $\begin{array}{l}\text { Zabrnie Górne near } \\
\text { Tarnobrzeg }\end{array}$ & EB60 & $\begin{array}{l}\text { N } 50^{\circ} 36^{\prime} \\
\text { E } 21^{\circ} 51^{\prime}\end{array}$ & ootheca & ecotone; clearing & $\begin{array}{l}\text { dry sandy grassland } \\
\text { Corynophoretum }\end{array}$ & $\uparrow$ \\
\hline 131 & $\begin{array}{l}\text { Zachwiejów ad } \\
\text { Tarnobrzeg }\end{array}$ & EA48 & $\begin{array}{l}\text { N } 50^{\circ} 25^{\prime} \\
\text { E } 21^{\circ} 33^{\prime}\end{array}$ & ootheca & $\begin{array}{l}\text { open; fallows } \\
\text { among farmland }\end{array}$ & $\begin{array}{l}\text { dry sandy grassland } \\
\text { /segetal }\end{array}$ & $\uparrow$ \\
\hline 132 & Zaklików Piachy I & EB72 & $\begin{array}{l}\text { N } 50^{\circ} 44^{\prime} \\
\text { E } 22^{\circ} 06^{\prime}\end{array}$ & ootheca & $\begin{array}{l}\text { ecotone; forest } \\
\text { edge/fallow }\end{array}$ & $\begin{array}{l}\text { dry sandy grassland } \\
\text { Corynophoretum }\end{array}$ & $\stackrel{\infty}{\leftrightarrow}$ \\
\hline 133 & Zaklików Piachy II & EB72 & $\begin{array}{l}\text { N } 50^{\circ} 44^{\prime} \\
\text { E } 22^{\circ} 05\end{array}$ & $\begin{array}{l}\pi, \text { P } \\
\text { ootheca }\end{array}$ & $\begin{array}{l}\text { ecotone; small } \\
\text { clearings }\end{array}$ & Calluno-Arctostaphylion & $\stackrel{\infty}{\leftrightarrow}$ \\
\hline 134 & $\begin{array}{l}\text { Zaklików military } \\
\text { training ground }\end{array}$ & EB72 & $\begin{array}{l}\text { N } 50^{\circ} 44^{\prime} \\
\text { E } 22^{\circ} 04^{\prime}\end{array}$ & 9 & $\begin{array}{l}\text { ecotone; large } \\
\text { clearing }\end{array}$ & $\begin{array}{l}\text { heath/dry sandy } \\
\text { grassland }\end{array}$ & $\infty$ \\
\hline 135 & $\begin{array}{l}\text { Zaosie Obojna near } \\
\text { Stalowa Wola }\end{array}$ & EB60 & $\begin{array}{l}\text { N } 50^{\circ} 36^{\prime} \\
\text { E } 21^{\circ} 57^{\prime}\end{array}$ & ootheca & open; fallow lands & Calluno-Nardetum & $\uparrow$ \\
\hline 136 & $\begin{array}{l}\text { Zapolednik near } \\
\text { Stalowa Wola }\end{array}$ & EB60 & $\begin{array}{l}\text { N } 50^{\circ} 33^{\prime} \\
\text { E } 21^{\circ} 56^{\prime}\end{array}$ & 9 & open; fallow lands & $\begin{array}{l}\text { dry sandy grassland } \\
\text { Corynophoretum }\end{array}$ & $\leftrightarrow$ \\
\hline 137 & $\begin{array}{l}\text { Zatyki for. range ad } \\
\text { Nisko }\end{array}$ & EA79 & $\begin{array}{l}\text { N } 50^{\circ} 28^{\prime} \\
\text { E } 22^{\circ} 05^{\prime}\end{array}$ & 9 & $\begin{array}{l}\text { ecotone; forest } \\
\text { track }\end{array}$ & $\begin{array}{l}\text { heath/dry sandy grassland } \\
\text { Corynophoretum }\end{array}$ & $\stackrel{\infty}{\leftrightarrow}$ \\
\hline 138 & $\begin{array}{l}\text { Zbydniów near } \\
\text { Tarnobrzeg }\end{array}$ & EB60 & $\begin{array}{l}\text { N } 50^{\circ} 38^{\prime} \\
\text { E } 21^{\circ} 56^{\prime}\end{array}$ & ootheca & $\begin{array}{l}\text { ecotone; forest } \\
\text { edge/fallow }\end{array}$ & $\begin{array}{l}\text { dry sandy grassland } \\
\text { /meadow }\end{array}$ & $\uparrow$ \\
\hline 139 & $\begin{array}{l}\text { Żdżary near Sędziszów } \\
\text { Małopolski }\end{array}$ & EB45 & $\begin{array}{l}\text { N } 50^{\circ} 08^{\prime} \\
\text { E } 21^{\circ} 38^{\prime}\end{array}$ & ڤ & open; fallow lands & $\begin{array}{l}\text { dry sandy grassland } \\
\text { /meadow }\end{array}$ & $\uparrow$ \\
\hline \multicolumn{8}{|c|}{ Wyżyna Lubelska (The Lubelska Uppland) } \\
\hline 1 & & EB54 & $\begin{array}{l}\mathrm{N} 50^{\circ} 53^{\prime} \\
\mathrm{E} 21^{\circ} 51^{\prime}\end{array}$ & 9 , ootheca & $\begin{array}{l}\text { open; } \\
\text { gravel-pit }\end{array}$ & $\begin{array}{l}\text { dry sandy grassland } \\
\text { /ruderal }\end{array}$ & $\uparrow ?$ \\
\hline 2 & Annopol II & EB54 & $\begin{array}{l}\text { N } 50^{\circ} 54^{\prime} \\
\text { E } 21^{\circ} 51^{\prime}\end{array}$ & ootheca & $\begin{array}{l}\text { ecotone; forest } \\
\text { margin/fallow }\end{array}$ & $\begin{array}{l}\text { meadow/dry sandy } \\
\text { grassland / segetal }\end{array}$ & $\uparrow$ \\
\hline 3 & $\begin{array}{l}\text { Chruślanki } \\
\text { Mazanowskie }\end{array}$ & EB65 & $\begin{array}{l}\mathrm{N} 51^{\circ} 01^{\prime} \\
\mathrm{E} 21^{\circ} 55\end{array}$ & 9 , ootheca & $\begin{array}{l}\text { ecotone; forest } \\
\text { margin/fallow }\end{array}$ & $\begin{array}{l}\text { dry sandy grassland } \\
\text { Corynophoretum }\end{array}$ & $\uparrow$ \\
\hline 4 & Gościeradów Folwark & EB63 & $\begin{array}{l}\text { N } 50^{\circ} 52^{\prime} \\
\text { E } 21^{\circ} 56^{\prime}\end{array}$ & ootheca & $\begin{array}{l}\text { ecotone; forest } \\
\text { margin/fallow }\end{array}$ & $\begin{array}{l}\text { dry sandy grassland } \\
\text { Corynophoretum }\end{array}$ & $\uparrow ?$ \\
\hline 5 & Kopiec near Annopol & EB54 & $\begin{array}{l}\text { N } 50^{\circ} 55^{\prime} \\
\text { E } 21^{\circ} 50^{\prime}\end{array}$ & ootheca & open; fellow lands & $\begin{array}{l}\text { dry sandy grassland } \\
\text { Corynophoretum }\end{array}$ & $\uparrow$ \\
\hline 6 & $\begin{array}{l}\text { Modliborzyce near } \\
\text { Janów Lubelski }\end{array}$ & EB92 & $\begin{array}{l}\text { N } 50^{\circ} 46^{\prime} \\
\text { E } 22^{\circ} 21^{\prime}\end{array}$ & 9, ootheca & open; fallow lands & $\begin{array}{l}\text { mesoxerothermic/ dry } \\
\text { sandy grassland }\end{array}$ & $\uparrow ?$ \\
\hline 7 & $\begin{array}{l}\text { Nieszawa near } \\
\text { Józefów }\end{array}$ & EB55 & $\begin{array}{l}\text { N } 51^{\circ} 01^{\prime} \\
\text { E } 21^{\circ} 50^{\prime}\end{array}$ & ootheca & open; fallow lands & $\begin{array}{l}\text { dry sandy grassland } \\
\text { Corynophoretum }\end{array}$ & $\uparrow$ \\
\hline 8 & $\begin{array}{l}\text { Opoka Duża near } \\
\text { Annopol }\end{array}$ & EB63 & $\begin{array}{l}\text { N } 50^{\circ} 52^{\prime} \\
\text { E } 21^{\circ} 53^{\prime}\end{array}$ & $\begin{array}{l}\text { juv., } \\
\text { ootheca }\end{array}$ & open; fallow lands & $\begin{array}{l}\text { dry sandy grassland } \\
\text { Corynophoretum }\end{array}$ & $\uparrow$ \\
\hline 9 & $\begin{array}{l}\text { Piotrawin near Opole } \\
\text { Lubelskie }\end{array}$ & EB56 & $\begin{array}{l}\text { N } 51^{\circ} 07^{\prime} \\
\text { E } 21^{\circ} 48^{\prime}\end{array}$ & ootheca & $\begin{array}{l}\text { fallow among } \\
\text { farmlands }\end{array}$ & $\begin{array}{l}\text { dry sandy grassland } \\
\text { /rudera }\end{array}$ & $\uparrow$ \\
\hline 10 & $\begin{array}{l}\text { Wymyslów near } \\
\text { Annopol }\end{array}$ & EB63 & $\begin{array}{l}\mathrm{N} 50^{\circ} 51^{\prime} \\
\mathrm{E} 21^{\circ} 55^{\prime}\end{array}$ & ootheca & $\begin{array}{l}\text { open; fallows } \\
\text { among farmland }\end{array}$ & $\begin{array}{l}\text { dry sandy grassland } \\
\text { Corynophoretum }\end{array}$ & $\leftrightarrow$ \\
\hline \multicolumn{8}{|c|}{ Wyżyna Malopolska (The Malopolska Uppland) } \\
\hline $\begin{array}{l}1 \\
2\end{array}$ & $\begin{array}{l}\text { Bronisławów near } \\
\text { Ożarów } \\
\text { Chańcza near Raków }\end{array}$ & $\begin{array}{l}\text { EB44 } \\
\text { EB01 }\end{array}$ & $\begin{array}{l}\text { N } 50^{\circ} 57^{\prime} \\
\text { E } 21^{\circ} 41^{\prime} \\
\text { N } 50^{\circ} 39^{\prime} \\
\text { E } 21^{\circ} 04^{\prime}\end{array}$ & $\begin{array}{l}9, \text { ootheca } \\
9 \text {, ootheca }\end{array}$ & $\begin{array}{l}\text { ecotone; forest } \\
\text { edge/fallow } \\
\text { ecotone; forest } \\
\text { clearing }\end{array}$ & $\begin{array}{l}\text { dry sandy grassland } \\
\text { Corynophoretum } \\
\text { dry sandy grassland } \\
\text { Corynophoretum } \\
\end{array}$ & $\uparrow$ \\
\hline
\end{tabular}




\begin{tabular}{|c|c|c|c|c|c|c|c|}
\hline 1 & 2 & 3 & 4 & 5 & 6 & 7 & 8 \\
\hline 3 & $\begin{array}{l}\text { Czajków Południowy } \\
\text { near Staszów }\end{array}$ & $\mathrm{EB} 20$ & $\begin{array}{l}\text { N } 50^{\circ} 34^{\prime} \\
\text { E } 21^{\circ} 17\end{array}$ & ootheca & $\begin{array}{l}\text { ecotone; forest } \\
\text { edge/fallow }\end{array}$ & $\begin{array}{l}\text { dry sandy grassland } \\
\text { /segetal }\end{array}$ & $\uparrow$ \\
\hline 4 & $\begin{array}{l}\text { Duranów near Ostrowiec } \\
\text { Świętokrzyski }\end{array}$ & EB44 & $\begin{array}{l}\text { N } 50^{\circ} 58^{\prime} \\
\text { E } 21^{\circ} 38^{\prime}\end{array}$ & q, ootheca & $\begin{array}{l}\text { ecotone; forest } \\
\text { edge/fallow }\end{array}$ & $\begin{array}{l}\text { dry sandy grassland } \\
\text { Corynophoretum }\end{array}$ & $\uparrow$ \\
\hline 5 & $\begin{array}{l}\text { Koprzywnica near } \\
\text { Sandomierz }\end{array}$ & EB40 & $\begin{array}{l}\text { N } 50^{\circ} 35^{\prime} \\
\text { E } 21^{\circ} 33\end{array}$ & q, ootheca & $\begin{array}{l}\text { ecotone; forest } \\
\text { edge/fallow lands }\end{array}$ & $\begin{array}{l}\text { dry sandy grassland } \\
\text { Corynophoretum }\end{array}$ & $\uparrow$ \\
\hline 6 & $\begin{array}{l}\text { Kozłówek near } \\
\text { Ożarów }\end{array}$ & EB44 & $\begin{array}{l}\text { N } 50^{\circ} 59^{\prime} \\
\text { E } 21^{\circ} 42^{\prime}\end{array}$ & q, ootheca & $\begin{array}{l}\text { ecotone; forest } \\
\text { edge/fallow }\end{array}$ & $\begin{array}{l}\text { dry sandy grassland } \\
\text { Corynophoretum }\end{array}$ & $\uparrow$ \\
\hline 7 & $\begin{array}{l}\text { Krzemionki near } \\
\text { Ostrowiec Świętokrzyski }\end{array}$ & EB34 & $\begin{array}{l}\text { N } 50^{\circ} 58^{\prime} \\
\text { E } 21^{\circ} 30^{\prime}\end{array}$ & ootheca & $\begin{array}{l}\text { thin bushes/ } \\
\text { grasslands }\end{array}$ & $\begin{array}{l}\text { dry sandy/ xerothermic } \\
\text { grassland }\end{array}$ & $\uparrow$ \\
\hline 8 & $\begin{array}{l}\text { Loniów near } \\
\text { Sandomierz }\end{array}$ & EB30 & $\begin{array}{l}\text { N } 50^{\circ} 33^{\prime} \\
\text { E } 21^{\circ} 30^{\prime}\end{array}$ & 甲 & $\begin{array}{l}\text { ecotone; forest } \\
\text { edge/meadow }\end{array}$ & meadow (fresh meadow) & $\uparrow$ \\
\hline 9 & $\begin{array}{l}\text { Maksymilianów near } \\
\text { Ostrowiec Świętokrzyski }\end{array}$ & EB34 & $\begin{array}{l}\text { N } 50^{\circ} 59^{\prime} \\
\text { E } 21^{\circ} 29^{\prime}\end{array}$ & 9 , ootheca & $\begin{array}{l}\text { large clearing, } \\
\text { fallow lands }\end{array}$ & $\begin{array}{l}\text { dry sandy grassland } \\
\text { Corynophoretum }\end{array}$ & $\uparrow$ \\
\hline 10 & $\begin{array}{l}\text { Sudoł I near Ostrowiec } \\
\text { Świętokrzyski }\end{array}$ & EB34 & $\begin{array}{l}\text { N } 50^{\circ} 57^{\prime} \\
\text { E } 21^{\circ} 28^{\prime}\end{array}$ & ootheca & open; fallow lands & $\begin{array}{l}\text { dry sandy grassland } \\
\text { Corynophoretum }\end{array}$ & $\uparrow$ \\
\hline 11 & $\begin{array}{l}\text { Sudoł II near Ostrowiec } \\
\text { Świętokrzyski }\end{array}$ & EB34 & $\begin{array}{l}\text { N } 50^{\circ} 58^{\prime} \\
\text { E } 21^{\circ} 27\end{array}$ & 9 , ootheca & ecotone; clearing & $\begin{array}{l}\text { dry sandy / xerothermic } \\
\text { grassland /ruderal }\end{array}$ & $\uparrow$ \\
\hline 12 & $\begin{array}{l}\text { Wiązownica Kolonia } \\
\text { near Staszów }\end{array}$ & EB20 & $\begin{array}{l}\mathrm{N} 50^{\circ} 34^{\prime} \\
\mathrm{E} 21^{\circ} 23\end{array}$ & 甲 & $\begin{array}{l}\text { ecotone; forest } \\
\text { edge/fallow }\end{array}$ & $\begin{array}{l}\text { dry sandy grassland } \\
\text { Corynophoretum }\end{array}$ & $\uparrow$ \\
\hline 13 & $\begin{array}{l}\text { Zimnowoda near } \\
\text { Staszów }\end{array}$ & EB11 & $\begin{array}{l}\text { N } 50^{\circ} 38^{\prime} \\
\text { E } 21^{\circ} 14^{\prime}\end{array}$ & $\begin{array}{l}\text { juv., } \delta, P, \\
\text { ootheca }\end{array}$ & $\begin{array}{l}\text { ecotone; forest } \\
\text { edge/fallow }\end{array}$ & $\begin{array}{l}\text { mesoxerothermic-dry } \\
\text { sandy grassland }\end{array}$ & $\uparrow$ \\
\hline \multicolumn{8}{|c|}{ Polesie Lubelskie } \\
\hline 1 & Załucze Stare & FB49 & $\begin{array}{l}\mathrm{N} 51^{\circ} 23^{\prime} \\
\mathrm{E} 23^{\circ} 06^{\prime}\end{array}$ & ootheca & open; fallow lands & $\begin{array}{l}\text { dry sandy/meadow with } \\
\text { young growth }\end{array}$ & intro? \\
\hline \multicolumn{8}{|c|}{ Karpaty (Carpathiens) } \\
\hline $\begin{array}{l}1 \\
2\end{array}$ & $\begin{array}{l}\text { Ciechania near } \\
\text { Krempna } \\
\text { Cisna }^{6}\end{array}$ & $\begin{array}{l}\text { EV37 } \\
\text { EV95 }\end{array}$ & $\begin{array}{l}\text { N } 49^{\circ} 26^{\prime} \\
\text { E } 21^{\circ} 30^{\prime} \\
\text { N } 49^{\circ} 14^{\prime} \\
\text { E } 22^{\circ} 19^{\prime}\end{array}$ & $\begin{array}{l}\text { juv. } \\
q\end{array}$ & $\begin{array}{l}\text { ecotone; forest } \\
\text { edge/grassland } \\
\text { town's street }\end{array}$ & $\begin{array}{l}\text { heath/mesoxerothermic } \\
\text { grassland }\end{array}$ & $\begin{array}{l}\uparrow \\
\downarrow\end{array}$ \\
\hline
\end{tabular}

Data from sources other than the author's field research, i.e. recent publications, questionnaires filled in by forest inspectorates, or personal written or verbal information, are presented in Table 3. As in the other tables, localities are given first, followed by UTM and/or geographic coordinates, sometimes the character of the environment, information source, and remarks. The author tried to verify unpublished information personally, especially in the case of the questionnaires and other unpublished reports. For this reason, some data presented in this table are similar (but never identical) to these in Table 2. This also concerns the results obtained at individual localities together with authors of some publications (Kata 2004, Sępiol 2005).

During the field research, the praying mantis was recorded from 165 sites, constituting $30 \%$ of total number of about 550 localities in the above mentioned regions. But we have a greater number of localities inhabited by the praying mantis if we add new data from sources other than own exploration, i.e, recent publications, questionnaires filled in the forest inspectorates, or personal written or verbal information (Table 3). The author tried to verify personally a great part of unpublished information, especially in the case of the questionnaires. For this reason, some data presented in both tables are similar (but never identical). This also concerns the results obtained at individual sites together with authors of some publications (Kata 2004, Sepiol 2005). If we exclude the localities that are repeated or negatively verified, we have a total number of 208 localities actually known as the sites of Mantis religiosa.

\footnotetext{
${ }^{6}$ Based on a specimen captured by dr Adam Stroiński (MIZ PAS).
} 
Table 3. Localities of Mantis religiosa after new publications, questionnaire of surveys and verbal communications

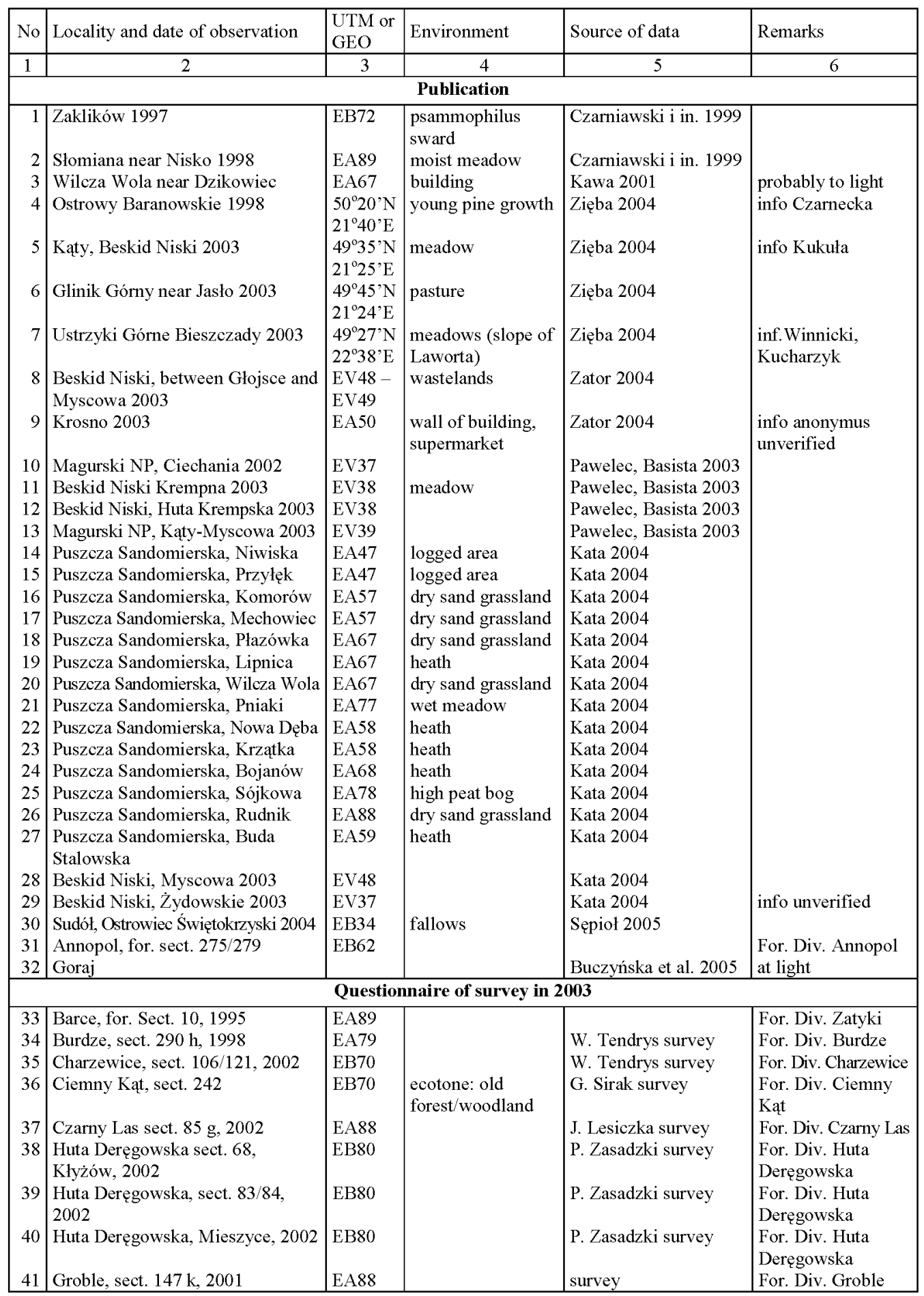




\begin{tabular}{|c|c|c|c|c|c|}
\hline 1 & 2 & 3 & 4 & 5 & 6 \\
\hline 42 & Klapówka, sect. 222/223/209, 2002 & EA66 & & survey & For. Div. Klapówka \\
\hline 43 & Maziarnia, sect. 167 & EB78 & & M. Waniewski survey & \\
\hline 44 & Przyłęk sect. 41, 2002 & $\mathrm{EA} 47$ & & survey & For. Div. Przyłęk \\
\hline 45 & $\begin{array}{l}\text { Rudnik Stróża cemetery next sect. } \\
27 \text { of Borowina }\end{array}$ & EA89 & & A. Nicalek survey & For. Div. Borowina \\
\hline 46 & Zapolednik, sect. $180,1995-1996$ & EB60 & & R. Dudziak survey & $\begin{array}{l}\text { For. Div. } \\
\text { Zapolednik }\end{array}$ \\
\hline 47 & Zatyki sect. 15 h, 1997 & EB78? & & survey & For. Div. Zatyki \\
\hline \multicolumn{6}{|c|}{ Personal communications } \\
\hline 48 & Huta Krzeszowska near Biłgoraj & FB00 & & $\begin{array}{l}\text { K. Daniłowicz } \\
\text { Targowiska }\end{array}$ & info unverified \\
\hline 49 & Pólsieraków near Harasiuki & FA09 & clearings & $\begin{array}{l}\text { K. Danilowicz } \\
\text { Targowiska }\end{array}$ & info unverified \\
\hline 50 & Sieraków near Harasiuki & FA09 & clearings & $\begin{array}{l}\text { K. Danilowicz } \\
\text { Targowiska }\end{array}$ & info confirmed \\
\hline 51 & Pietrusza Wola near Krosno 2003 & EA51 & meadow & L. Luczaj & $\begin{array}{l}\text { foto; info not } \\
\text { confirmed }\end{array}$ \\
\hline 52 & $\begin{array}{l}\text { Zawadka Rymanowska near } \\
\text { Rymanów } 2004\end{array}$ & EV58 & meadow & L. Luczaj & foto \\
\hline 53 & Korzenno near Raków 2006 & EB01 & meadow & anonymus info & info unverified \\
\hline 54 & $\begin{array}{l}\text { Puszcza Sandomierska: Poręby } \\
\text { Dymarskie }\end{array}$ & EA57 & & anonymus info & info unverified \\
\hline 55 & $\begin{array}{l}\text { Kunów near Ostrowiec } \\
\text { Świętokrzyski } 2006\end{array}$ & EB14 & $\begin{array}{l}\text { highway (dead } \\
\text { specimen) }\end{array}$ & B. Sępioł & $\begin{array}{l}\text { verbal } \\
\text { communication }\end{array}$ \\
\hline 56 & $\begin{array}{l}\text { Rudka Baltowska near Ostrowiec } \\
\text { Świętokrzyski } 2006\end{array}$ & EB35 & & B. Sępioł & $\begin{array}{l}\text { verbal } \\
\text { communication }\end{array}$ \\
\hline 57 & $\begin{array}{l}\text { Dunale near Ostrowiec } \\
\text { Świętokrzyski } 2005\end{array}$ & EB35 & & anonymus info & info unverified \\
\hline 58 & Głogów Małopolski 2006 & EA65 & garden & worker of Forest Office & info unverified \\
\hline 59 & Urszulin near Wlodawa & FB59 & garden & $\begin{array}{l}\text { worker of Poleski } \\
\text { National Park }\end{array}$ & $\begin{array}{l}\text { negative } \\
\text { verification }\end{array}$ \\
\hline 60 & $\begin{array}{l}\text { Załucze Stare - Spławy, Poleski } \\
\text { NP }\end{array}$ & FB49 & educational path & B. Piotrowski & $\begin{array}{l}\text { negative } \\
\text { verification }\end{array}$ \\
\hline 61 & Bogdanka near Łęczna & FB48 & mine slag & anonymus info & $\begin{array}{l}\text { negative } \\
\text { verification }\end{array}$ \\
\hline
\end{tabular}

\section{Discussion}

\section{Present distribution of mantis}

According to the most recent data, the praying mantis inhabits parts of the following regions of south-eastern Poland: Wyżyna Małopolska, Wyżyna Lubelska, Kotlina Sandomierska and Karpaty Zachodnie (Figs 2 and 3). Apart from that, the species has been recorded from one locality in Polesie Lubelskie (Załucze Stare) but this information should be treated with great reserve because the insect may have been introduced recently (see afterwards). Therefore, the present range of the praying mantis in Poland consists of two parts, i.e. "the Sandomierz area" and "the Carpathian area".

The Sandomierz region is an insular area. It mainly consists of the central part of Kotlina Sandomierska, i.e. so called Puszcza Sandomierska (which includes Równina Tarnobrzeska, Plaskowyż Kolbuszowski and eastern part of Płaskowyż Tarnowski) and the north-western part of Równina Biłgorajska (Lasy Lipskie, Lasy Janowskie and western part of Puszcza Solska). The newly discovered localities allow inclusion of the north-western part of Plaskowyż Tarnogrodzki in the main part of Sandomierz area. In Puszcza Sandomierska, Lasy Lipskie and Lasy Janowskie, the occurrence of $M$. religiosa is permanent. Documented information goes back to the early 1920s and the species occurs there even today. Also the largest number of 
localities have recently been recorded from this central part of the Kotlina Sandomierska, i.e. 139 of the total number of 165 localities reported from Poland in general (Table 2).

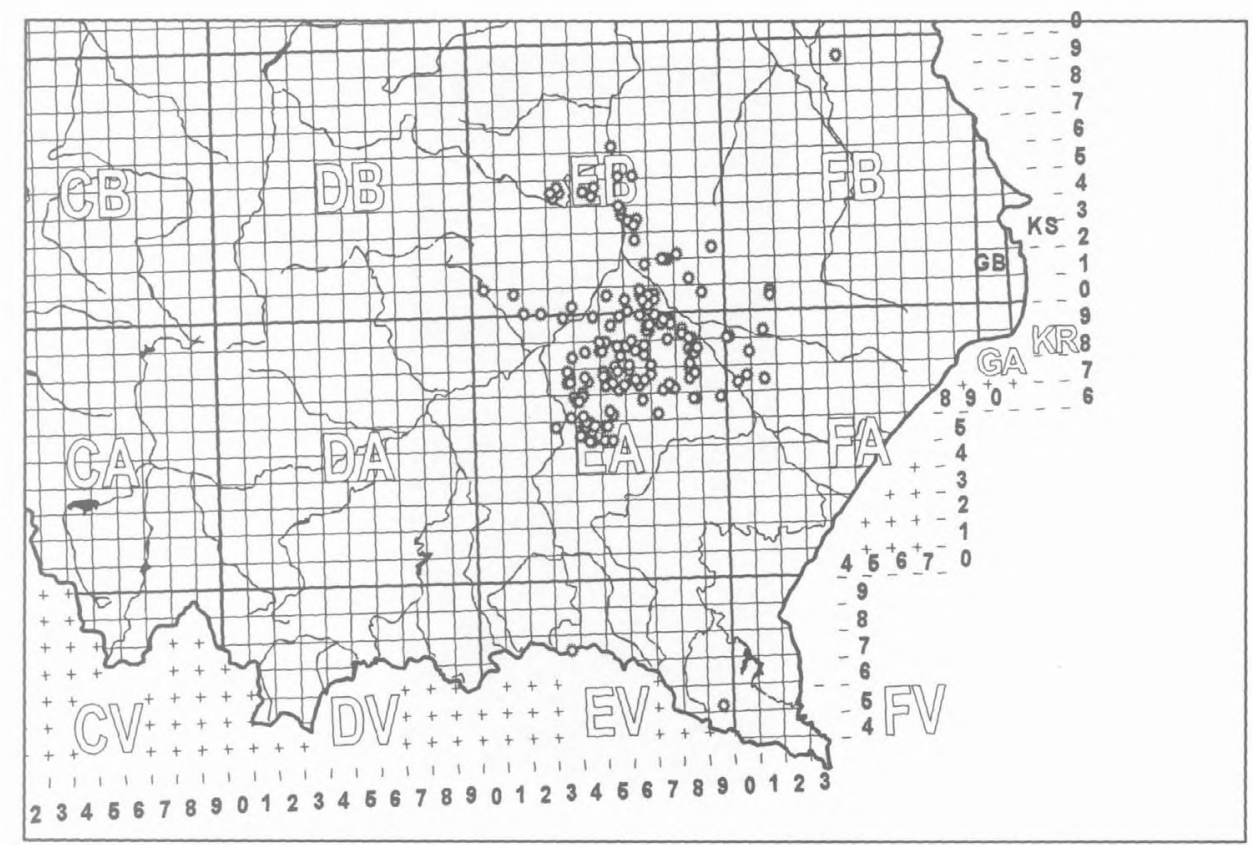

Fig. 2. The sites of Mantis religiosa (L.) in south-eastern Poland in the backgrounds of the UTM grid.

Parts of Wyżyna Lubelska and Wyżyna Malopolska belonging to "the Sandomierz area" of the occurrence of the praying mantis are adjacent to the main part described above and seem to be strongly dependent on it. In Wyżyna Malopolska, M. religiosa occurs in Przedgórze Ilżeckie, Pogórze Szydlowskie and Niecka Polaniecka. However, two localities can be considered to belong to Wyżyna Sandomierska (Łoniów and Koprzywnica - bordering on Niecka Polaniecka). A historical locality - Góry Wysokie was situated in Wyżyna Sandomierska too but the occurrence of $M$. religiosa has not been confirmed there for over 40 years. Other historical localities in Wyżyna Małopolska have not recently been confirmed too. They include Skarżysko in Góry Świętokrzyskie (the Świętokrzyskie Mts.) and Chotel Czerwony in Dolina Nidy (the Nida River Valley).

In Wyżyna Lubelska, the praying mantis was recorded from two mesoregions: Wzniesienia Urzędowskie (6 localities), which borders on Równina Bilgorajska of the south, and Kotlina Chodelska (with one, presumably new locality in Piotrawin). Dzierzkowice, also located in Wzniesienia Urzędowskie, was probably wrongly marked on the map of Bazyluk (1977) paper as a locality of the praying mantis. An ephemeral locality in Modliborzyce-Lucieniec (Liana 1997) was situated on a slope of the Sanna River valley and bordered on Wzniesienia Urzędowskie and Roztocze Zachodnie. The only locality in Roztocze that has been documented so far (Buczyńska et al. 2006) is on the border of Roztocze Zachodnie and Kotlina Bilgorajska. It is very likely that the male of $M$. religiosa, caught at night in Goraj, came from the locality in Lasy Janowskie. The specimen was caught at night, before a storm, when the temperature was very high. In such conditions, activity of the males is increased and they fly greater distances. 


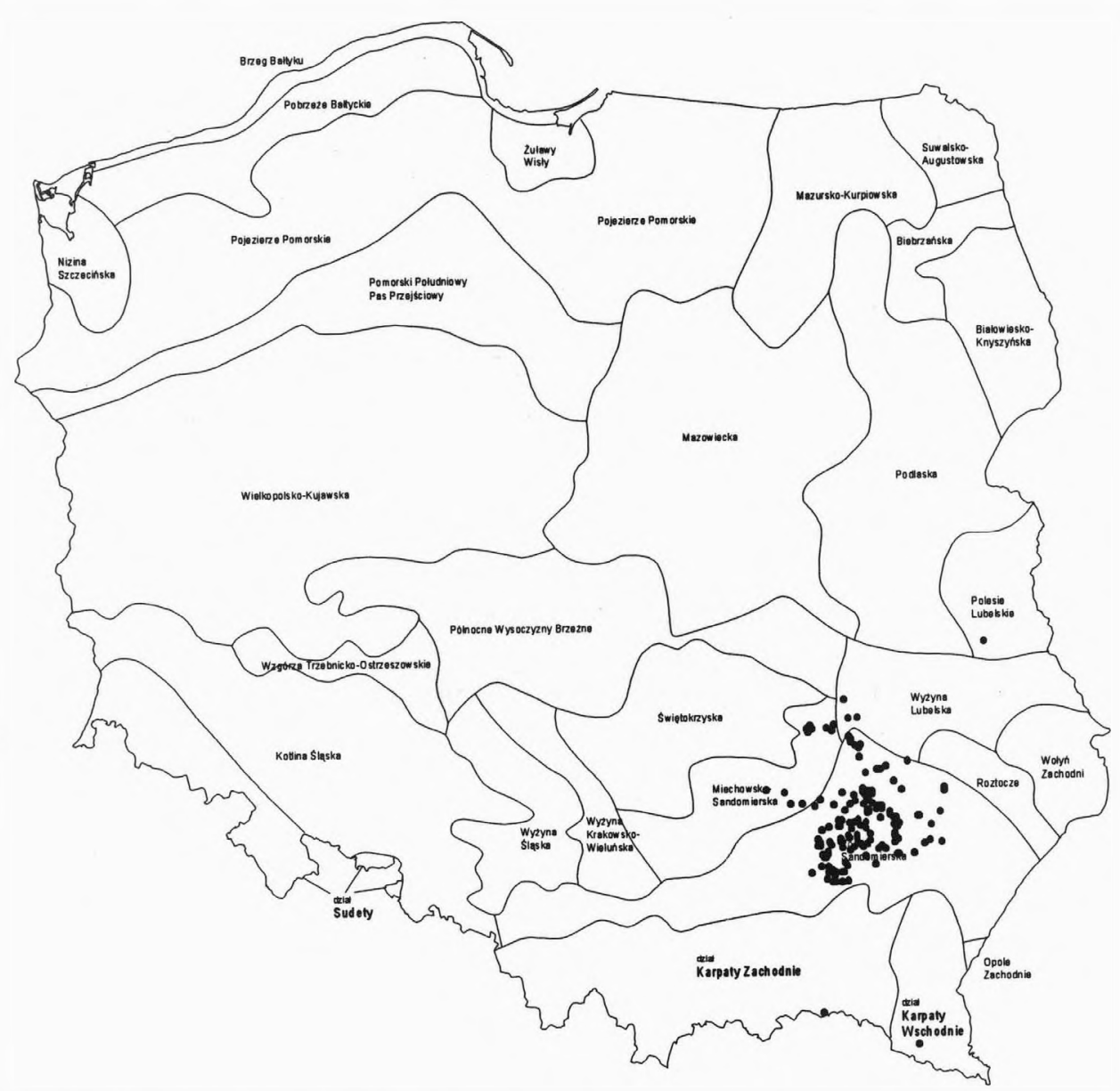

Fig. 3. Present sites of Mantis religiosa (L.) in south-eastern Poland in the background of regional division.

The distribution of M. religiosa in the Polish Carpathians is probably ephemeral and seems to be strongly dependent on populations inhabiting N Slovakia, where stable populations have been known from at least 1992 (Thomka 2003). Moreover, its durability seems to depend on weather conditions in subsequent years. Except for Beskid Niski and Pogórze Środkowobeskidzkie, Carpathian localities can hardly form one Carpathian area because the locality in Cisna (the Bieszczady Mountains) is isolated from the locality in Ustrzyki Dolne (Pogórze Przemyskie). Each of these localities can be of a different origin (provided that they were inhabited by spontaneous dispersion and not deliberate introduction).

Previously (historical data in Table 1) $M$. religiosa was recorded only once from Polish part of Western Carpathians, i.e. from Nowa Góra in Pieniny. Apparently, several specimens were caught there at one time. However, since then, the occurrence of the praying mantis has never been confirmed either in that locality or in the whole region. At the beginning of the $1970 \mathrm{~s}$, faunistic team research was conducted in Pieniny; W. Bazyluk (orthopterologist) explored these mountains for many years. At present, they are continuously monitored by many 
specialists since there is a national park there. The record from Nowa Góra can, therefore, be regarded as an incidental effect of dispersion from Slovakian populations.

In Beskid Niski, M. religiosa was first observed in 2002 and then at a few localities in 2003 (Pawelec \& Basista 2003). In 2004, it was found only in one locality but development of the specimens was retarded to such an extent that there was no chance of reaching maturity before winter (nymphs of 3rd-4th instar in September). In 2005, M. religiosa was not found in any locality.

A similar situation was probably in eastern part of Beskid Niski (Lipowiec and Zawadka Rymanowska near Jaśliska) where only nymphs were also found in September, 2004. A similar delay in the development was observed in northern Slovakia near Svidnik in 2004 but adult insects and oothecae were observed simultaneously with nymphs. Two $M$. religiosa records from Pogórze Środkowobeskidzkie (one of them published) are from 2003. In the same year, the species was recorded from Bieszczady; one record was based on verbal information and documented by a specimen from Cisna and the other was published by Zięba (2004) from the vicinity of Ustrzyki Dolne. The first locality was near Polish-Slovak border and Przelęcz Łupkowska. At the turn of the 1960s and the 1970s, a large team was conducting faunistic research in Bieszczady over several years. If there had been stable localities of the praying mantis there, it would have been impossible to miss them. So, these localities are undoubtedly new and ephemeral.

The locality in Polesie Lubelskie is isolated and lying far from the nearest ones in Wyżyna Lubelska (Chruślanki Mazanowskie) and in Roztocze Zachodnie (Goraj) (nearly $100 \mathrm{~km}$ ). It is very likely that $M$. religiosa has recently been introduced there, perhaps even deliberately. In such a situation, this locality should not be taken into account when defining range limits of the species in Poland. However, when considering the possible reasons for and ways of recent expansion of the species, it should be a valuable contribution.

To sum up. the range limits of $M$. religiosa of "the Sandomierz" insular area are as follows: northern limit - Maksymilianów and Kozlówek near Ostrowiec Św., Piotrawin near Opole Lubelskie $\left(51^{\circ} 07 \mathrm{~N}\right.$ the most northern locality) and Chruślanki Mazanowskie near Kraśnik; western limit - Maksymilianów and Chańcza near Raków $\left(21^{\circ} 04^{`} \mathrm{E}\right.$, the most western locality $)^{7}$; eastern limit - Goraj (the most eastern locality), Nadrzecze and Sól near Bilgoraj; southern limit - Dąbrowica Duża, Jelna near Leżajsk, Czarna Sędziszowska $\left(50^{\circ} 08^{\prime} \mathrm{N}\right.$, the most southern locality) and Podole near Przeclaw. Pietrusza Wola near Krosno (ephemeral locality?) is the most northern locality in the Carpathian area. Altitudinal range extends up to $600 \mathrm{~m}$ approximately (in Beskid Niski).

It can be calculated on a basis of map with UTM grid and the sites marked on it (Figs 1 and 2) that the area of insular range of the praying mantis is about $5000 \mathrm{~km}^{2}$. The centre - most compact and constant part of the distribution - is made up of Puszcza Sandomierska and the adjacent parts of Lasy Janowskie, Lasy Lipskie and Puszcza Solska with a total area of about $4000 \mathrm{~km}^{2}$.

\section{Habitat preference}

According to the data presented in Table 2, ecotonal sites (usually a few or several dozen metres from a wall of forest) or partially open sites constitute over $70 \%$ of all sites recorded during recent research. There are mainly new, unstable biotopes (fallow lands and wastelands) with dry sandy grassland. They are often situated in the areas which are defined as agricultural land because several years ago or even more recently they were really cultivated. Traces of balks and patches as well as a considerable admixture of ruderal and segetal species in plant

\footnotetext{
${ }^{7}$ Not including the unverified site in Korzenno near Raków, $20 \mathrm{~km}$ farther to the west.
} 
communities occurring in the form of sandy grassland with representatives of the order Corynophoretalia canescentis at various succession stages are the evidence of former cultivation. The composition of plant communities is very diverse and, in most cases, it may even be impossible to recognize their phytosociological category. Low tuft grasses, especially the grey hair-grass (Corynephorus canescens) and low rosette plants, e.g. Hieracium pilosella are the common physionomic characteristics of these environments. Together with bryophytes and lichens, they cover the poorest habitat areas. Depending on the age of fallow land, way of their earlier cultivation and type of the habitat, other grasses may locally predominate, e.g. of the genera Festuca, Calamagrostis or Agrostis. In these assemblages, patches of higher perennials, e.g. Hypericum sp., Rumex sp., Oenothera sp., are frequently used by the praying mantis as hiding places. The proximity of the forest wall makes local climate more favourable for thermophilous species.

Woodland sites, located in relatively small clearings with predominance of communities of Callunetum type or a mosaic of heather, pinewood undergrowth and dry sand grassland patches, also play an important role although their percentage is much lower (about $21 \%$ ). At these localities, coniferous forest species, e.g. all species of the genus Vaccinium sp. (including $V$. uliginosi in marshy coniferous forests) form a significant part of plant cover. The sites of this type can be defined as classic because Bazyluk $(1960,1977)$ considered them to be typical for the subspecies $M$. religiosa polonica, described by himself. Due to the character of vegetation (mosaic of heather and psammophilous grass patches), open sites in dunes areas on a military military training ground in Nowa Dęba should also be regarded as classic. The southern exposure of dune slopes can probably be the reason for the similarity of the microclimate to that under the influence of forest wall.

The finding of the praying mantis in moors and meadows of the humid type is a relatively new phenomenon in Poland. Habitats of this kind make up about $7 \%$ of all new records. But we must distinguish between a phenomenon of ineffective migration (when single migrants may appear in a completely inappropriate habitat as a high peat bog Imielty Lug) and expansion of the species to the environment which dries because of land melioration or lower rainfall. In Puszcza Sandomierska, several localities of M. religiosa in the moist meadows with Juncus effusus have been recorded (e.g. Grochowe II, Gwoździec III, Karolówka, Pniaki, Szydlowieckie Łaki). The drier the meadow, the more xerophilous or even xerothermic insects appear that the mantis can prey on. Phaneroptera falcata also appears there (Grochowe II, Pniaki), apart from extremely hygrophilous species of the order Orthoptera which still live in such a locality, e.g. besides Conocephalus dorsalis, Omocestus viridulus and Stethophyma grossum.

During recent research, no typical xerothermic sites of the praying mantis (with typical grasses and other plants) have been recorded although they were searched for in Wyżyna Małopolska (several sites in the lower Nida valley, Wyżyna Sandomierska and Przedgórze Iłżeckie, respectively) as well as in Wyżyna Lubelska and Roztocze. Chotel Czerwony (Nida valley) is the only historical Polish locality of this type (Witkowski 1992).

The sites of M. religiosa in Polish Carpathians are similar to those in northern Slovakia. For example, a site in Ciechania (Magurski National Park) resembled one of the localities in Svidnik (partly because it was located on a slope) and a locality in Čertižne near Medžilaborce in which vegetation was similar (heathland and meadows with mixture of thicket consisting of Juniperus communis, Rosa sp. and Berberis vulgaris). Meadows and pastures were mentioned most frequently in newly published reports concerning localities of the praying mantis in Beskid Niski, Bieszczady and Pogórze Strzyżowskie (Table 3). The meadows in Pietrusza Wola and in Lipowiec near Jaśliska had dense and rich vegetation consisting of a great number 
of species (verbal information provided by $Ł$. Łuczaj and the author's personal observations made during the search of the site in Pietrusza Wola).

\section{Character of populations and their trends}

The population types of the praying mantis listed below are, in fact, subsequent developmental stages of metapopulation structure which is typical of many rare species occurring at the limits of their range. Due to favourable changes of environmental conditions (warming, increase in potential habitat areas), many individuals migrating beyond the current limits of the old insular range or from "the population-source" can give rise to new "peripheral" populations. Some of these populations are of particularly ephemeral character, while others could become permanent components of a growing metapopulation.

As has been noted, the assessment of population trends in a given locality is a subjective diagnosis based on a number of phenomena concerning the environment and the population itself. The author decided to make such a diagnosis in the hope that, in the future, together with the knowledge of real changes, it may help to determine the ways of and reasons for dispersion of $M$. religiosa more precisely. Glowaciński et al. (1980, Table 1), characterized in a synthetic way the status of all Polish species of vertebrates and some invertebrates, including $M$. religiosa. The above mentioned table inspired the present author to take the trend into account.

Durable stable populations (trend $\infty$ ). Stable populations which have probably occurred in the same localities for several dozen years include seven populations existing in Kotlina Sandomierska, mainly in the area of Puszcza Sandomierska and on the military training grounds: Cietrzewiec I, II, III, Dąbrowskie Góry, Radomyśl, Wilcza Góra (in Nowa Dęba), Zaklików. All these localities are used in a similar way but with various intensity. They are ecotonal (big woodland clearing) or open environments (in vicinity of a forest complex) in areas covered with dunes. Due to that, the succession of forest is slow and the microclimate is optimal for thermophilous insects. It can be confirmed by the occurrence of such Orthopteran species as Phaneroptera falcata, Ephippiger ephippiger (last one only in Radomyśl), Calliptamus italicus, Stenobothrus nigromaculatus, Stauroderus scalaris, Psophus stridulus, and other invertebrates, e.g. Scolia hirta (Hymenoptera), Eresus niger (Aranaea), etc. A mosaic of vegetation (large patches of heathland, psammophilous grassland, peat bogs situated among dunes, clumps of trees, patches of pine and mixed forest) provides hiding places for nymphs and adults of the praying mantis. Apart from that, it is the place of development, feeding and shelter for many various insects which constitute food sources for $M$. religiosa.

According to a transect survey carried out in 1996 and in 2005 on the military training ground in Nowa Dęba, population density of the praying mantis was low (one specimen/200 $\mathrm{m}^{2}$ or one specimen $/ 300 \mathrm{~m}^{2}$ in the localities Cietrzewiec I and Cietrzewiec III). If the density was identical in the whole area, the total size of the military training ground population could be several dozen thousand. In fact, high concentration areas of Mantis can be observed in some patches, e.g. near the peat bog in Cietrzewiec and near Dąbrowskie Góry. In optimal years (the year 1996 was exceptionally cold and cannot be regarded as optimal), the number of specimens in stable populations on military training grounds can amount as many as five or six thousand. Such populations must cause expansion of the praying mantis to adjacent areas. At present, objects are used in such a way that, in most areas, succession of forest communities is slower and, as a result, durability of $M$ religiosa populations is guaranteed.

Durable mobile populations ( $\infty$ and $\leftrightarrow$ ). It is evident from historical data that these populations are very durable although their dynamics is noticeable due to the fact that they inhabit areas in depth of forests. Their continuous movements over short distances are caused by continuous environmental changes. Forest management practices support or even enforce forest succession, restricting the environment suitable for the praying mantis. On the other 
hand, forest felling and wood harvest expand these types of environment. Logging areas, young cultures, small clearings around forest settlements, forest complex edges, cuttings and woodless strips of land along power lines are colonized by Mantis, most often with the help of man who unconsciously contributes to it (wood and seedlings transport from one site to other, etc.). In Puszcza Sandomierska, there are many such classic forest sites which, due to that, exist for many, sometimes even for several dozen years. The sites in Borów, Barce, Nisko and Rudnik forestries have been known since the 1920s. The site in Dęba Rozalin has been known since the 1950s and the one in Krzatka has probably existed since the 1920s or so. Although there has been slight movement of some localities, their permanent existence is unquestionable. 28 out of 165 localities (Table 2) were included in this category (which is about 17\%).

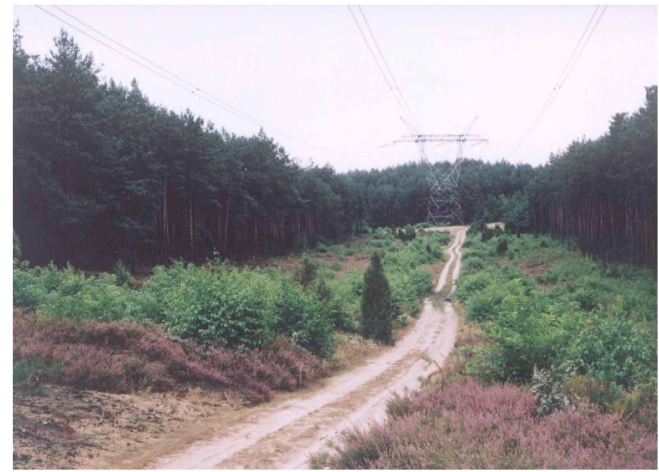

Fig. 4. Classic locality of Mantis religiosa - a clearing along a power line in the forest complex of Kosowy forestry district.

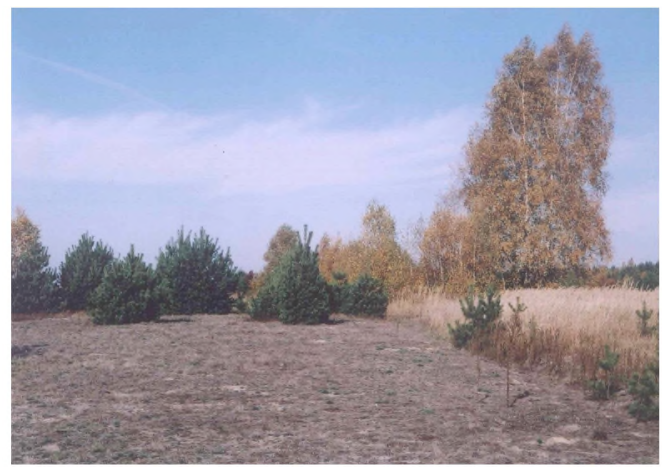

Fig. 6. A new site of Mantis religiosa in Niwiska near Kolbuszowa - follow lands with a mosaies of dry sand grassland, wet meadow and segetal vegetation

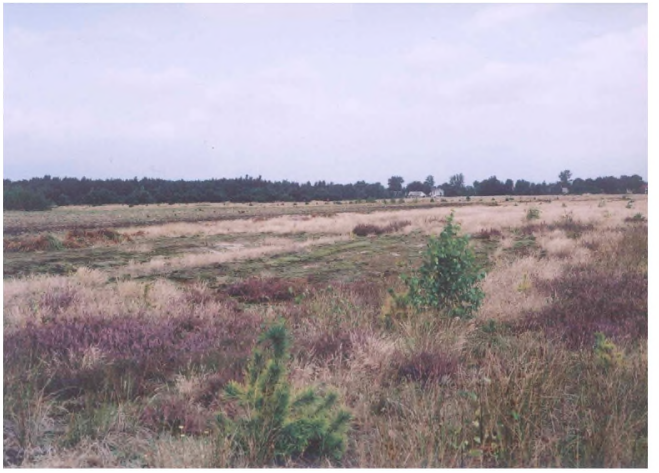

Fig. 5. The ecotonal site of Mantis religiosa in Wilcza Wola Zmystow - follow lands on the forest edge with heath and dry sand grassland.

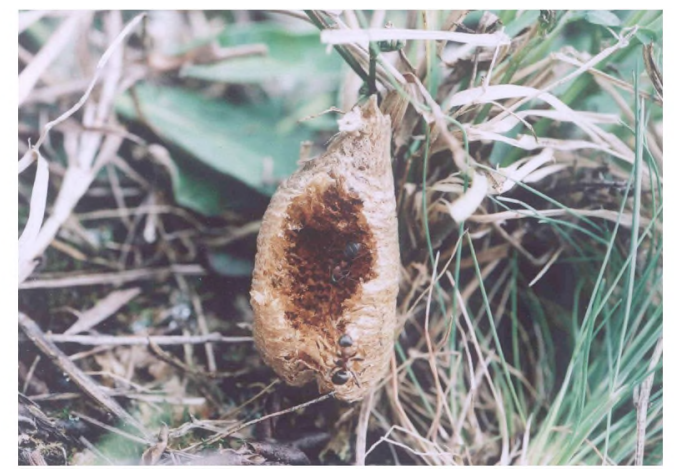

Fig. 7. Devastation of Mantis religiosa ootheca caused by ants Formica rufibarbis.

The site "Kosowy forestry" is an interesting example. It is situated under a power line in a woodless strip of land about $40 \mathrm{~m}$ wide and running across a pinewood complex in sand dune areas between Ługnica and Szydlowiec (Fig. 4). On the slopes of the dunes there was an Arctostaphylo-Callunetum community with quite expansive bearberry, bilberry and cranberry, much heather and cup-mosses prevailing in the areas in which main species were absent. The population density of mantis is relatively low there (Table 4), the population seems to be stable 
(it has been observed since 1999) and probably can even strengthen newly emerging migrant populations, e.g. in Ługnica and Przylęk Marmury. For fire protection, forest succession is constantly suppressed under the power lines, which guarantees $M$. religiosa populations relative stability.

In the past, $M$ religiosa could exist in our country mainly thanks to this classic type of sites and populations. At present, their role and significance constantly decrease which may pose a threat to the existence of this species in the future.

Migrant populations (trend $\leftrightarrow$ ). They can be described as intermediate between the mobile and pioneer populations and as the first phase of expansion. Their developmental trend oscillates between growth and decrease of population size and between expansion and regression of the species. They inhabit ecotonal, semi-open localities on forest complex edges and, as a result, they are exposed to negative changes of the climate more than the forest populations (Fig. 5) They are derived from stable military training ground populations or mobile forest ones and often form parts of a great local metapopulation which can meet each other and which live in various types of environment (e.g. Burdze I - Burdze V localities, Table 2). Almost any complex includes, among others, populations from classic (i.e. forest) ones. In recent years (which have been warm and dry) the migrant populations have also colonized mat-grass meadows and humid meadows with rush - Juncus effusus. 48 populations (29\%), including 46 ones in Kotlina Sandomierska, were considered to be migrant. Many of them were found in the vicinity of Nowa Dęba military training ground.

Pioneer populations (trend $\uparrow$ ). At present, there is a size growth trend in these populations. They inhabit new localities in unstable environment of secondary character, i.e. fallow lands which have existed for less than 20 or even for just a few years. Most often, psammophilous grasslands of the order Corynophoretalia form the main plant communities there. It is often difficult to identify such an assemblage due to the mixture of plants and high percentage of ruderal and segetal elements (including expansive kenophytes) (Fig. 6). Young age of the fallow lands and isolation of a given locality from other ones, especially those whose longer existence has been proved, are convincing evidence of new expansion of the praying mantis.

In Puszcza Sandomierska, the date of appearance of pioneer population in a given locality can sometimes be determined with reasonable accuracy. In Wólka Niedźwiedzka, near Marynin forester's house, M. religiosa appeared not until as in 2003 or 2004. Previous research in this locality repeatedly indicated that it could not have existed before. A similar date may also be suggested for a nearby site - Krasne Wólka Niedźwiedzka. In site "Jelna" near Leżajsk, the population was discovered in autumn 2003 only on the basis of a few oothecae. Next year, the number of the oothecae increased more than ten times. But despite a suitable environment, the praying mantis was not found in the vicinity of Leżajsk except this only one locality. Absence of $M$. religiosa until 2005 in many other searched sites on the southern boundary of Plaskowyż Kolbuszowski (vicinity of Żołynia, Głogów Małopolski, Dębica) confirms the recent age of species expansion to the south.

Pioneer populations constitute $40 \%$ (67 sites) of all the populations recorded in the whole research area, probably including 100\% in Wyżyna Małopolska, 80\% in Wyżyna Lubelska and only $35 \%$ in Kotlina Sandomierska.

Vanishing populations (trend $\downarrow$ ). A decreasing trend associated with unfavourable environmental changes was observed in three sites of $M$. religiosa. In Agatówka and Rozwadów (on the periphery of Charzewice forestry divisions) the localities are vanishing due to strong anthropopression, i.e. development of residential suburbs in Stalowa Wola agglomeration. The site Jamnica I disappeared following artificial afforestation with pine 
crops. Only some years ago in 1996-1997, it was a large, sunny clearing with sites of many thermophilous grasshoppers, as well as of Mantis religiosa and of the smooth snake (Coronella austriaca). Young pines shaded a large part of the clearing and changed the vegetation type in the whole area. In 2003, not a single specimen of the praying mantis was found there.

Ephemeral populations (trend $\uparrow$ ). Most often, ephemeral populations are evidence of dispersion to the environment or regions that are not suitable for a given species. They can also be the result of accidental or intentional introduction by man. Probably that kind of ephemeral population was observed for two years in Modliborzyce (Liana 1997). After several years, the ephemeral population collapses and the species vanishes but it may appear again later. Carpathian populations, especially from Magurski National Park, seem to be a good example. Carpathian sites in Poland are probably constantly colonized by specimens from Slovakian populations which expand northwards. In a variable and severe mountain climate, durability of such new populations must be short timed.

Migrant specimens (trend $\uparrow$ ). The occurrence of single specimens not associated with the presence of a praying mantis population in a given locality is evidence of migration. Several such cases were recorded among other: in Chyly (a part of Stalowa Wola) and Górno near Sokołów Małopolski (males were probably attracted to light in buildings) and in Żdżary (a single male in the environment which was potentially suitable for the praying mantis). A female found in Cisna was probably a migrant specimen too (unless it was introduced). The measurements of this female are considerably larger than the maximum ones given for the females of the subspecies M. religiosa polonica Bazyluk, 1960 (Bazyluk 1960, 1977). Due to the proximity of the Przelęcz Łupkowska (Lupków Mountain Pass), it was possible that specimens of $M$. religiosa religiosa migrated from Slovakia to southern parts of the Bieszczady.

Species introduction ("intro"). During the research, the praying mantis was deliberately introduced twice by the author in the compact "Sandomierz" area. After hatching in laboratory, nymphs were introduced in the region from which the oothecae were collected, mainly in the same localities which the oothecae came from, but also in two specified sites in Puszcza Sandomierska considered to be earlier "mantis-free". In Huta Przedborska Katy near Kolbuszowa, three attempts of introduction were made - in 2004, 2005 and 2006. Each time, more and more nymphs were released in a sandy wasteland in the forest. In 2004, about 200 nymphs were released, whereas in 2006, the approximate number amounted to 1500 . In August 2005, a few adult insects were also introduced in this locality. The population lived there for a short time and only single specimens were able to survive. In autumn 2006, not a single ootheca was found and, as a result, the introduction is considered to be unsuccessful.

An introduction in a nearby site in Poręby Huciskie, made in 2006, was different from the latter. About 250 nymphs were released in 0.5 hectare of fallow land. The locality was checked several times; one adult female and a small number of developing nymphs were found. During another inspection, in October that year, one old and two new oothecae were found which may be the evidence of earlier, spontaneous colonization. Despite the previous assessment, the locality may, therefore, not have been „mantis-free”.

On the basis of field research, the site in Zahucze Stare in the Polesie National Park protection zone was considered to be the result of a recent accidental or even intentional (illegal) introduction; research in nearly 30 other sites gave negative results and proved it to be totally isolated in the Polesie region. The nearest localities in Wyżyna Lubelska are situated almost $100 \mathrm{~km}$ from that one. Because oothecae from the previous years were not found, the introduction might have concerned nymphs or adults. In the future, genetic research could help in explaining the origin of the "Zahucze" population. 


\section{Abundance of the praying mantis}

In both versions of "The Polish Red Data Book of Animals" an estimated abundance of $M$. religiosa in Poland was given. Regarding the situation in 2003-2006, the estimates of both $Z$. Witkowski and A. Liana were too low. Witkowski (1992) thought that in Poland there were about 20 populations of the praying mantis consisting, in total, of a few hundred or a few thousand specimens. Liana (2004) supposed that in optimal years, the total number of specimens in about 40 populations might be even a dozen thousand.

A transect method was used to count the praying mantis in 1997 and 2005 on the military training ground in Nowa Dęba. Counts were carried out in a few sites near the Cietrzewiec peat bog. According to the results, the density was approximately 0.3 per $100 \mathrm{~m}^{2}$, i.e. about 30 specimens per one hectare. On the military training ground, the praying mantis forms a metapopulation consisting of small, scattered populations which meet with each other, mainly because of a relatively good flight capability of the males. Assuming that the military training ground subpopulations inhabit only $10 \%$ of the area of the whole object, i.e. about $180-200$ hectares, their size is not smaller than 5.5 or 6 thousand specimens and may be even larger, perhaps up to a dozen thousand during more favourable years. At present, it is certainly the largest concentration of $M$. religiosa in Poland.

The remaining populations are much smaller. However, there are many such populations (over 130 are known only from Kotlina Sandomierska and over 200 from the whole country). The total number of the specimens in these populations is certainly many times as large as that in the military training ground population. To sum up, at present, in optimal climate conditions, the total size of mantis population in Poland may even amount to several dozen thousand specimens.

Density studies were conducted in a few localities and at fixed plots using the method of quadrates (Table 4). The density of imagines was generally checked in August, that of the oothecae - in late autumn (October, November), i.e. when few adult insects (mainly females) were still alive.

Table 4. Density of Mantis religiosa populations at selected sites in Puszeza Sandomierska; - - not recorded in standard investigetions; 0 - not recorded in intensive investigations.

\begin{tabular}{|c|c|c|c|c|}
\hline $\begin{array}{c}\text { Sites, } \\
\text { total surface area, }\end{array}$ & Control date & Imagines $/ 100 \mathrm{~m}^{2}$ & Oothecae $/ 100 \mathrm{~m}^{2}$ & Remarks \\
\hline Kosowy for., $400 \mathrm{~m}^{2}$ & $\begin{array}{c}2004 \\
\text { (Sep) } 2005 \\
2006 \\
\end{array}$ & $\begin{array}{c}0,5 \\
1 \\
1 \\
\end{array}$ & $\begin{array}{l}1 \\
2 \\
2 \\
\end{array}$ & density stable \\
\hline Niwiska II, ST plot, $500 \mathrm{~m}^{2}$ & $\begin{array}{l}2004 \\
2005 \\
2006\end{array}$ & $\begin{array}{l}3 \\
\mathrm{n} \\
\mathrm{n}\end{array}$ & $\begin{array}{c}13 \\
7 \\
7\end{array}$ & $\begin{array}{c}\text { seasonal dynamic of } \\
\text { density }\end{array}$ \\
\hline Niwiska II, PB plot, $2000 \mathrm{~m}^{2}$ & $\begin{array}{c}2005 \\
\text { (Aug, Nov) 2006 }\end{array}$ & $\begin{array}{l}n \\
3 \\
\end{array}$ & $\begin{array}{c}10 \\
5\end{array}$ & density unstable \\
\hline Pniaki, $100 \mathrm{~m}^{2}$ & $\begin{array}{c}\text { (Sep) } 2003 \\
\text { (Aug, Sep) } 2004 \\
2005\end{array}$ & $\begin{array}{c}2 \\
13 \\
0\end{array}$ & $\begin{array}{c}10 \\
2 \\
0\end{array}$ & $\begin{array}{l}\text { density very unstable - } \\
\text { high risk of damage }\end{array}$ \\
\hline Rudnik Doliny $600 \mathrm{~m}^{2}$ & $\begin{array}{c}2004 \\
\text { (Aug, Nov) } 2005 \\
\text { (Oct) } 2006\end{array}$ & $\begin{array}{l}\mathrm{n} \\
3 \\
\mathrm{n}\end{array}$ & $\begin{array}{l}6 \\
6 \\
6 \\
\end{array}$ & density rather stable \\
\hline
\end{tabular}

After conversion of the results into a larger area, the density will range from 50 or 100 specimens per one hectare (Kosowy) to 300 specimens per 1 hectare. The density is, therefore, similar to that at the sites from southern Slovakia (Šušlik 1995). It should be noted that the distribution of the praying mantis in Niwiska II site (whose total area exceeds 100 hectares) is irregular. The site consists of narrow, private allotments which lay fallow or are cultivated to a 
various extent. For such sites, we can give only a rough estimate of the abundance of the praying mantis.

An analy sis of the data presented in Table 4 shows that prognoses of population durability and abundance of the praying mantis on the basis of density of the oothecae must be made with some caution. Low density of oothecae can guarantee stability of the populations of relatively low density, e.g. in a classic, forest site Kosowy, first recorded in 1999. The population in Rudnik Doliny, observed since 1996 (but metapopulation in Rudnik has existed at least since 1920) seems to be stable. During the last three years, population density has been studied systematically in the total area of 6 plots. The situation in Niwiska is less stable, especially on a PB plot where the number of oothecae in 2005 was twice as high as in 2006. The population in Pniaki was so unstable that in 2005 the observations were discontinued because no oothecae were found.

Another phenomenon that should be noted is a disproportionately low density of imagines in relation to the potential number of their offspring. Assuming an average number of 100 nymphs from one ootheca ${ }^{8}$, potential number of $1 \mathrm{st}$ instar nymphs in Rudnik (area $600 \mathrm{~m}^{2}$ ) could amount to 3600 . However, the average density of adult specimens in August 2005 was $3 / 100 \mathrm{~m}^{2}$, i.e. about $0.5 \%$ of the potential density of the nymphs! Such a considerable reduction results from very high mortality rate of $M$. religiosa during ontogenesis, including embryonic stages. The greatest damage in this developmental period is probably caused by ants from the genera Formica and Tetramorium (see Threats, discussed below). The populations are reduced to a relatively small extent by the parasitoids Podagrion pachymerum (Walk.)

\section{Notes on phenology}

Changes of phenology associated with weather condition variability can affect abundance, dispersion and survival of many species. According to recent observations, the duration of various developmental stages of $M$. religiosa has extended and the life cycle has changed because some nymphs hatch from the oothecae in autumn (Table 5).

Table 5. Phenology of Mantis religiosa; stage: - embryos, $\mathbf{a}$-nymphs, $\mathbf{0}$-imagines; ? - duration of stage (last year embryos) not included

\begin{tabular}{|c|c|c|c|c|c|c|c|c|c|c|c|c|}
\hline Author & I & II & III & IV & $\mathrm{V}$ & VI & VII & VIII & IX & $X$ & XI & XII \\
\hline $\begin{array}{l}\text { Bazyluk } 1977, \\
\text { Poland }\end{array}$ & 000 & 000 & 000 & 000 & $\begin{array}{c}000 \\
\mathbf{0}\end{array}$ & $\begin{array}{l}\text { o?? } \\
\text { qם }\end{array}$ & $\begin{array}{l}? ? ? \\
\text { QDQ? }\end{array}$ & $\begin{array}{c}? ? ? \\
\text { Doo } \\
\text { 0,0] }\end{array}$ & $\begin{array}{l}000 \\
\text { 000 }\end{array}$ & $\begin{array}{l}000 \\
\text { 00? }\end{array}$ & 000 & 000 \\
\hline $\begin{array}{l}\text { Liana } 2007, \\
\text { Poland }\end{array}$ & 000 & 000 & 000 & 000 & $\begin{array}{l}000 \\
\text { D00 }\end{array}$ & $\begin{array}{l}\text { o?? } \\
\text { qua }\end{array}$ & $\begin{array}{c}? ? ? \\
\text { dod } \\
0\end{array}$ & $\begin{array}{c}? 00 \\
\mathbf{0} \\
000\end{array}$ & $\begin{array}{c}000 \\
\mathbf{a}^{9} \\
000 \\
\end{array}$ & $\begin{array}{c}000 \\
\mathbf{0}^{10} \\
000\end{array}$ & $\begin{array}{c}000 \\
0 \\
0\end{array}$ & 000 \\
\hline $\begin{array}{l}\text { Voisin 2003, } \\
\text { France }\end{array}$ & & & & & & ספם & $\begin{array}{c}\text { dod } \\
\text { व }\end{array}$ & बסוס & ס & Dी0 & & \\
\hline
\end{tabular}

The most important changes in phenology of Polish populations of $M$. religiosa that have been observed include earlier emergence of nymphs from oothecae in spring (the earliest date was 1 May), longer survival of imagines, i.e. until the beginning of November, hatching of some nymphs in September or October in the year in which the oothecae were deposited.

\footnotetext{
${ }^{8}$ Ehrmann (1984) determined the number of eggs in an ootheca at 60-70 and Detzel \& Ehrmann (1998) according Tauscher have given 114 eggs as maximum. The present author saw up to as many as 180 nymphs in laboratory cultures.

${ }^{9}$ Nymphes of the first generation have been observed in September in the Carpathiens only.

${ }^{10}$ Emerging of the second generation has been observed from autumn 2005.
} 
The latter phenomenon had never been observed in Central Europe and it is one of population reducing factors in $M$. religiosa. Particularly numerous cases of such an acceleration of emergence were recorded in 2006. In Niwiska on a PB plot, emergence marks (traces of the first moult) were observed on one ootheca of 21 October. During a detailed inspection of the same area in November, the total number of 108 oothecas was recorded (in the area of 2000 $\mathrm{m}^{2}$ ). Traces of emergence were observed on 6 of them.

\section{Threats and natural enemies}

Before the beginning of the 21 st century, when expansion of the praying mantis was noticed in Poland, reduction of restricted habitats suitable for this species was the main threat. At present, it seems to be not so important because the praying mantis readily colonizes other types of environment (fallow lands with dry sand grassland). If fallow areas continue to grow during the next few years, further expansion of $M$ religios $a$ will be possible provided that the climate remains unchanged. There is little likelihood that fallow land will regain its agricultural function but after several years, its area will shrink as a result of intentional afforestation or succession (which can already be observed in the Podkarpacie province). In such a situation, dispersion of the mantis will be suppressed. The story of Jamnica I population is a very good example of possible course of events.

Ants of the genus Formica inhabiting sandy areas are the most dangerous natural enemies of $M$ religiosa populations. These ants occur in very sunny, ecotonal sites near the forest edge. Damage caused e.g. by Formica rufibarbis ${ }^{11}$ can be easily identified because the ants open the dorsal wall of the ootheca at the front and then meticulously dissect the eggs (Fig. 7). In autumn, some oothecae in many localities are more or less damaged. In November 2006, on ST plot in Niwiska $(500 \mathrm{~m} 2), 12$ out of 61 oothecae (almost $20 \%$ ) were strongly damaged. Five of them ( $8 \%$ ) were nearly destroyed. In the spring of $2006,24 \%$ of the oothecae were damaged by the ants in the same area. The day of Mantis emergence is particularly critical because the nymphs which sit together on the ootheca are sometimes attacked by the ants. The oothecae from which only some nymphs hatched are also exposed to aggressive attacks. Some oothecae are probably totally destroyed by the Formica.

Podagrion pachymerum does not constitute a very serious threat to Polish populations of the praying mantis. The parasitoid was recorded from 11 out of more than 165 localities but in 6 cases, its presence was recorded in laboratory while breeding the oothecae collected in the field. The proportion of attacked oothecae was not always the same. The highest percentage $(27 \%)$ was in the case of the oothecae from Grębów Zajezierze. A slightly lower percentage ( $20 \%$ of the oothecae) was recorded from Rudnik in 2005 during direct inspection in the field (characteristic openings are left after the emergence of the parasites). Only some eggs of the praying mantis are destroyed because one parasitoid develops at the expense of one embryo. The number of hatching parasitoids may sometimes be larger than the number of $M$. religiosa specimens and vice versa. Regarding one of the oothecae collected in site Sól, an identical success of $P$. pachymerum and $M$. religiosa was observed (over 70 nymphs of the praying mantis and the same number of parasitoids).

The participation of vertebrates in reduction of $M$ religiosa at its embryonic stage does not seem to be significant. Oothecae which were probably damaged (pecked) by birds were recorded only sporadically. Birds and lizards must catch older nymphs and imagines but this phenomenon has not been examined. Reproductivity of $M$. religiosa populations at their embryonic stage often collapses for unknown reasons. Such a phenomenon was observed in the field, e.g. on a plot BR in Niwiska site where the nymphs emerged only from some oothecae.

${ }^{11}$ The author thanks Dr. Wiesława Czechowska for determination of ant species. 
In breeding conditions, only a small number of nymphs emerged from the oothecae collected at some localities and some of them did not even have any trace of the emergence. The material collected from Sarnów in 2005 can be a good example. In spring 2006, the nymphs emerged only from $50 \%$ of these oothecae and only 35 nymphs, on average, hatched from one ootheca. Such a situation could be a result of extreme dehydration of the oothecae during autumn in the field.

\section{Distribution changes}

In central Europe Mantis religiosa is the only representative of Mantodea and reaches the northern limit of its range there. At the same time it is the most widely distributed species of the order. $M$ religiosa is legally protected in many countries because its distribution is scattered and it even seemed to have been regressing for some decades. In recent years, the growth in the area it occupies aroused more interest in this insect and it turned out that there were gaps in the knowledge of its biology, ecology and even world distribution (for instance incorrect information about the occurrence of $M$. religiosa in Australia). Also the origin of North American populations of this species has been explained only recently. The praying mantis was introduced from Europe to the state of New York in 1899 and then expanded to most states in north-western part of the USA and to the state of Ontario in Canada. In the state of Delaware, it inhabits old fields together with two other species of Mantodea which are of Asian origin, i.e. with Tendera sinensis Sauss. and T. angustipennis SAUss. All these species acclimatized very well and became the subject of comparative ecological studies (see e.g. Eisenberg \& Hurd 1993). Dispersion success of $M$. religiosa in North America is evidence of its great ecological flexibility despite high thermal requirements.

It is evident from an overview of historical data concerning Mantis religiosa in Germany (Brechtel, Detzel \& Ehermann 1996) that the distribution fluctuations are similar to those in Poland. First records also appeared in the 18th century and came from Frankfurt am Main, which was the northernmost historical site in Germany. This site had not been confirmed until the end of the 19th century. Information about the praying mantis from the 19th century is scanty; nearly all reliable information comes from Baden-Württemberg, i.e. from the most southern region which has been and is still a main centre of $M$. religiosa distribution in Germany (Detzel \& Ehrmann 1998). At present, Rheinland-Pfalz is the second distribution centre. First information from Rheinland-Pfalz appeared at the beginning of the 19th century but only in the 1990s was it recorded from many localities. Among 33 recently discovered German localities of the praying mantis and mentioned by Ehrmann (2003), 26 are known from Baden-Württemberg, 5 from Rheinland-Pfalz, one from Sachsen (15 km north of Leipzig), and one from Berlin, situated within city boundaries. 4 localities from Baden and 3 ones from Rheinland are considered by Ehrmann (2003) to be "autochthonous" and the remaining are new localities which exist thanks to climate conditions that are optimal for the praying mantis. The author expects further expansion.

One of the most enigmatic records of the praying mantis from Germany is a relatively small, isolated population inhabiting a railway siding in Berlin which has not been used for many years. Its site was discovered in 1998 and, since that date, has been permanently inspected (Berg \& Keller 2004). It covers the area of about 7.5 hectares $(500 \times 150 \mathrm{~m})$ and is probably the most northern of all the sites that are currently known in Europe.

In the area of former Czechoslovakia, until the $1980 \mathrm{~s}, M$. religiosa distribution was limited to southern Moravia and to southern and eastern regions of Slovakia (Červená Kniha 1992). At the end of the 1990s, the species appeared in many localities of Central Moravia (Chladek 1998, Piszkiewicz, Beneš \& Konvička 2000), and in Slovakia its range, actually, expanded to the whole country up to its most northern points (Krištin \& Mihál 2000, Vidlicka 2001, Lukáš 
\& Lukášová 2003, Thomka 2003). In the 1990s, growth of density and abundance among $M$. religiosa populations was also observed in Austria (Berg \& Zuna-Kratky 1997). Some expansion of the species was recorded even in France although prior to that, it had avoided some northern and western regions. Voisin (2003) notes that the praying mantis prefers open areas, including fallow lands.

\section{Reasons for expansion}

Global warming is most often considered to be the main cause of the current expansion of the praying mantis and many other species. Although climatologists argue about the reasons for the warming, they all agree that the intensity of this phenomenon has increased during last two decades. Regarding Poland, it can be seen e.g. from an analysis of meteorological data from a weather station in Warsaw. Data from a period of over 200 years were analyzed by Lorenc (2004). The author divided this period into several thermal stages which are worth mentioning due to potential correlation between historical fluctuations of the climate and the observed changes of $M$. religiosa distribution.

In the second half of the 18th century, there was a thermal optimum. In Warsaw, an average annual temperature was $7.8^{\circ} \mathrm{C}$. The optimum probably concerned all central Europe. First information about the occurrence of the praying mantis in Poland (vicinity of Warsaw) and Germany (vicinity of Frankfurt) appeared in that period. In Europe, low average annual temperatures maintained almost throughout the 19th century. In 1829, the average temperature was extremely low $\left(4.7^{\circ} \mathrm{C}\right)$. Lorenc $(2004)$ notes that in the first half of the 19th century, the atmosphere on the globe was heavily polluted for a long period due to more intensive volcanic activity. We do not have any information about $M$. religiosa in Poland from that period and information from Germany is rather scanty (Brechtel et al. 1996). A systematic rise in an average annual temperature was recorded in the period from 1890 to 1980 . It was particularly noticeable in 1911-1920 and 1931-1950. In 1980-2000 there was a dramatic increase in temperature and, as a result, an average annual temperature in Warsaw was $8.7^{\circ} \mathrm{C}$. The highest average annual temperature $\left(10.1^{\circ} \mathrm{C}\right)$ was recorded in 2000 . In the same year, extremely high average annual temperature was recorded e.g. in Kotlina Sandomierska $\left(9.5^{\circ} \mathrm{C}\right.$ in Rzeszów and $10^{\circ} \mathrm{C}$ in Tarnów).

For decades, the distribution of $M$. religiosa in central Europe was limited to the environments that were the most favourable in terms of temperature and to the warmest regions. In Poland, it was limited to central part of Kotlina Sandomierska and parts of the adjacent regions. A synthetic description of the climate in all regions of Poland (including Kotlina Sandomierska) based on the data from 1950-1970 can be found in a paper by J. M. Matuszkiewicz (2002). Among 36 lowland geobotanical regions, Kotlina Sandomierska was among the warmest ones with an average annual temperature of $7.8^{\circ} \mathrm{C}$, a growing season lasting 218 days (the number of days with an average temperature of more than $5^{\circ} \mathrm{C}$ ), the longest summer ( 107 days with an average temperature of more than $15^{\circ} \mathrm{C}$ ), and a high index of thermal continentalism (Ewert's index is 50.1). A degree days index reached the highest level in Poland, amounting to $1866^{\circ} \mathrm{C}$ for temperatures higher than $15^{\circ} \mathrm{C}$ and $3142^{\circ} \mathrm{C}$ for temperatures higher than $0^{\circ} \mathrm{C}$ (in both cases, only one region in Poland had higher degree days index).

On the basis of data from Rzeszów Jasionka weather station it can be concluded that during last 30 years there has been a tendency towards gradual increase in an average annual temperature in Kotlina Sandomierska. It dropped several times to less than $7^{\circ} \mathrm{C}$ or even to $6.5^{\circ} \mathrm{C}$ (especially between 1976 and 1985). In 1981-1990, an average annual temperature was $8.0^{\circ} \mathrm{C}$, in $1991-2000-8,2^{\circ} \mathrm{C}$ (and exceeded $9^{\circ} \mathrm{C}$ three times), and in 2001-2006 the temperature rose up to $8.5^{\circ} \mathrm{C}$. In 2002 , the temperature was $9.2^{\circ} \mathrm{C}$. According to Lorenc (2004), 
a tendency towards the increase in average annual temperature of the air (observed across Poland) is mainly caused by rises in winter. A negative tendency prevailed in summer until 2000. Warmer winters may also contribute to population size growth and, as a result, to more intensive dispersion of the praying mantis. Experiments done by Salt $\&$ James (1947) showed that a temperature of about $-20^{\circ} \mathrm{C}$ does not kill any diapausing embryos of the praying mantis and that a temperature of about $-30^{\circ} \mathrm{C}$ kills some of them $(15 \%)$. However, when they compared their results with field observations in the state of Ontario during the winter of $1945 / 1946$, they concluded that in natural conditions, a temperature of $-29^{\circ} \mathrm{C}$ could be lethal even for almost $90 \%$ of the embryos. According to Salt \& James (1947), the influence of low temperature on the embryos must have been strengthened by other factors which were not determined by these authors. In Kotlina Sandomierska, such low winter temperatures were recorded for the last time in 1985 (Rzeszów Jasionka, February, $-30.5^{\circ} \mathrm{C}$ ) and in 1987 (Rzeszów Jasionka, January, $-30.9^{\circ} \mathrm{C}$ ). Local conditions (topographic features, vegetation cover, thickness of snow cover) can protect the oothecae, at least in some localities. Constant range of the praying mantis in central part of Kotlina Sandomierska (whose climate is characterized by high index of continentality) is a confirmation of the fact that Polish populations of $M$. religiosa (subspecies $M$. religiosa polonica) are adapted to such conditions.

Classic localities of $M$. religiosa in pinewood clearings or on the edge of a coniferous forest (protected by a wall of forest) have a special microclimate which decreases the influence of continental climate in cold seasons of the year and increases it in warm ones. In the vegetation season, the best thermal conditions for thermophilous species are near the wall of forest, in northern parts of medium size clearings. Writing about the Kluczowody Valley, Durlo \& Wilczyński (2006) proved that zonal distribution of temperature was typical for forest clearings. North-eastern parts of the clearings have the highest average daily temperature and moderate amplitudes, and in these parts we can observe mostly mantis specimens and oothecae.

Due to climate warming, the role of classic forest sites as $M$. religiosa refuges has become less important. However, expansion of the species has been possible only because new types of environment appeared which were suitable for it. In Poland, this requirement has been met because during the last several years, a dramatic increase in wasteland and fallow lands area could be observed. According to data of Central Statistical Office (Grzesiak \& Domańska 2006), idle land and fallow land area has increased systematically in Polish agricultural land since 1990 and by 2002, it has increased 14 times (2300 thousand hectares). In 2000, fallows and idle lands covered the area of 140 thousand hectares in the Podkarpacie province, i.e. almost $22 \%$ of the total arable land area in the province and over $8 \%$ of the total idle land area in Poland. Since 2003, a decrease in idle land area has been observed but in 2005, they still constitute $18 \%$ of arable land in the Podkarpacie province, and the province is first in Poland in this respect. In sandy soil, fallows quickly become covered with communities of the order Corynophoretalia, together with segetal, ruderal or meadow elements, depending on currently adjacent areas and the former way of using the land. At present, about $60 \%$ of $M$. religiosa sites are situated in fallows and various types of wasteland. The populations associated with such an environment were defined as migrant or pioneer. More and more often, the praying mantis appears in open areas, far from a wall of forest which moderates the climate conditions. Stability and duration of such sites will probably depend on the character and longevity of climate oscillation in the future. Moreover, dry sand grasslands are not durable and their succession is relatively fast. In such an environment, $M$. religiosa populations must, therefore, be unstable. 


\section{Dispersal possibilities}

Active migration possibilities of $M$. religiosa seem to be very limited. Thus, it would be interesting to know the ways of its dispersion. It may be chiefly passive. Ehrmann (1985) in one of his earliest papers stated that $M$. religiosa is a sedentary species, associated with a specific environment. He had an opportunity to observe a very dense population of the praying mantis in the Spanish island of Mallorca for three months. Its density was 43 imagines per 100 $\mathrm{m}^{2}$ from beginning to end of observation. However, 10 years later, he and his co-authors (Brechtel, Ehrmann \& Detzel 1996) considered routes of $M$. religiosa expansion from southern regions of Germany to the north and possibilities for active protection of these natural routes. Discovery of the locality of $M$. religiosa in Berlin stirred up a lively discussion among specialists about possible ways of introducing it by man (Berg \& Keller 2004). All discussants agreed that the species could not have appeared there in a natural way.

The males of $M$. religiosa are capable of flying. When disturbed by man, they often fly over distances of more than ten metres. Chladek (1998) observed in Moravia the flight of males to a distance of fifty metres. On the other hand, the females most often hide among dense vegetation. During the author's investigation a flying female was observed only once, however the distance was not longer than 3-4 m. At night, single males are attracted to illuminated windows (Kawa 2001, and verbal information from Puszcza Sandomierska) or to light traps (Buczyńska et al. 2006). We can, therefore, conclude that flight capabilities of this species are variable, depending on a given specimen, and that these capabilities are fully used only in particular circumstances, e.g. during mating flight before a storm. Flying males probably play an important role in communication among local, isolated subpopulations. It is quite possible that some specimens in a given population show more morphological and physiological predisposition towards migration than others. A female caught in Cisna (Bieszczady) and probably originating from Slovakia could belong to such specimens. On the basis of the measurements, it was categorized as M. religiosa religiosa. Although the female had a medium body length, the length of its wings was nearly equal to the maximum for this subspecies. The mechanisms of development of solitary phases and long-winged specimens in Orthoptera are well known. The same factors may, perhaps, determine morphological changes and migration instinct in very dense populations of Mantodea, but this phenomenon has not been yet observed in the case of praying mantises.

Research on the praying mantis in Poland show that man's activity plays an important role in permanent maintenance of its wide range and in its dispersion which has recently been intensified. The dispersion rate is evidence of a direct, most often unconscious, participation of man in dispersion of various developmental stages. Among these stages, the oothecae are certainly carried by man most frequently. When stuck to grasses or perennials, they can be transported with hay for many kilometres. In the past, they were probably dispersed in such a way by soldiers. Some oothecae are deposited on small trees or shrubs and, as a result, they can be carried with seedlings. Single oothecae can also be found on timber collected in a forest and then transported to sawmills or building sites. In southern Europe, the oothecae are often deposited on stones. During a discussion about the origin of populations inhabiting railway areas in Berlin, the specialists considered their introduction with an aggregate used as track foundation (Berg \& Keller 2004).

The oothecae can be carried by wind but they are probably transported over relatively short distances only. In spring, many oothecae in open areas are detached and they are sometimes carried with small tufts of grass pulled out from the ground. Hurricanes could, of course, carry the oothecae over long distances, and such a phenomenon must occur in the United States where old fields cover very large areas. Transport of the oothecae by rivers, resulting in a successful dispersion of the praying mantis in Poland, does not seem to be very likely. Most 
often, our riversides are not suitable for this species. Theoretically, transport of the oothecae by birds may also play some role in dispersion of $M$. religiosa, but it is not known what species of birds eat egg cases of the Mantodea and in what quantity they do so.

Several hours after the emergence, nymphs begin to disperse. At that time, their tendency towards movement is probably the strongest. In many observed sites, attacks of ants accelerated nymph leaving the hatching place. If an ootheca was deposited in an open area with sparse tufts of grasses (as in plot PB of the site Niwiska II), the majority of the nymphs will leave this place and hide among vegetation. At the end of summer and in autumn, migration of imagines to more open fragments and sun-warmed sites is observed because it is easier for them to find potential prey there. However spontaneous, active migration of the nymphs and adult females over longer distances probably do not play a significant role in the dispersion of $M$ religiosa.

If a population is isolated and not too dense, migration of males over long distances may provide for durability of the whole metapopulation and its protection against inbreeding. The nymphs and adults can also be carried by man but the older a stage is, the more unlikely its inadvertent transport. When discussing the reasons for the current expansion of the praying mantis, we must also allow for the possibility of deliberate introduction. In the past, people also carried various species experimentally or for fun. Today, probably, genetic research can help in determining the origin of specimens or questionable populations.

\section{Problems of protection of Mantis religiosa}

Should the praying mantis be protected if its expansion is currently observed? If we were sure that the present stimulating factors would exist in the future (for many years), it would not be necessary to protect the praying mantis from extinction. But only man's activity will certainly increase in the future, the other factors may change radically even within a few years in a negative direction for the mantis. In a few years, the praying mantis may become a rare species again, even if this process is not radical and fast enough to lead to complete extermination of this insect.

Note that although this species is more common in neighbouring countries, it is protected everywhere. Lack of species conservation in Poland could arouse more interest in this insect among foreign collectors and, as a result, dramatically reduce the size of our populations. The praying mantis should be protected due to its unique taxonomic rank in Central Europe and also due to its unique scientific value. In addition, this large and easily recognizable insect could serve as an umbrella species in protected areas.

The most important thing for threatened invertebrates is habitats protection. The project of the Landscape Park of Puszcza Sandomierska (Sandomierz Forest) should be finalized to protect not only the praying mantis but also many rare species of plants and animals living in this area, including grasshoppers, spiders, snakes, many birds and several species of mammals. The presence of Mantis religiosa is only one of natural values of this object.

\section{ACKNOWLEDGEMENTS}

During my research I have been helped by many people to whom I want to express my sincere appreciation. I have received a great deal of kindness and help from inhabitants of the region, fellow entomologists, and colleagues from the Museum and Institute of Zoology in Warsaw. First of all I am very grateful to Mr Tomasz Huflejt, MSc (Eng.) in Forestry, for his help and advice during the field and laboratory research. I am indebted to managers and workers of all forest district offices in Puszcza Sandomierska for valuable information and all the kindness they showed me during the years of investigation.

I would like to express my gratitude for valuable remarks of the reviewers. I made use of them while preparing the final version of this paper. 


\section{REFERENCES}

BAŁUT S. 1953. Modliszka w Puszczy Sandomierskiej. Chrońmy Przyrodę Ojczysta, Warszawa, 9 (1): 31-34.

BAZYLUK W. 1947. Szarańczaki (Orthoptera, Saltatoria) okolic Zwierzyńca (Zamojszczyzna) Fragmenta Faunistica Musei Zoologici Polonici, Warszawa, 5: 123-137.

BAZYLUK W. 1955. Uwagi dotyczące zmian zachodzących i zaobserwowanych $w$ ostatnich latach $w$ obrębie ortopterofauny. Polskie Pimo entomologiczne, Wroclaw, 24, suppl. 2: 113-121.

BAZYLtK W. 1956. Karaczany - Blattodea. Modliszki - Mantodea. In: Klucze do oznaczania owadów Polski. IX-X. PWN, Warszawa, 11, $40 \mathrm{pp}$.

BAZYLUK W. 1957. Nowe dla Polski lub rzadsze gatunki z rzędów Blattodea, Mantodea, Orthoptera i Dermaptera. Fragmenta Faunistica, Warszawa, 7: 263-282.

BAZYLUK W. 1960. Die geographische Verbreitung und Variabilität von Mantis religiosa (L.) (Mantodea, Mantidae), sowie Beschreibungen neuer Unterarten. Annales Zoologici, Warszawa, 18: 231-272.

BAZYLUK W. 1976. Karaczany i modliszki. Blattodea et Mantodea. In: Katalog fauny Polski, 17, 1. PWN, Warszawa, $31 \mathrm{pp}$.

BAZYLUK W. 1977. Blattodea et Mantodea - karaczany i modliszki (Insecta). In: Fauna Polski, 6. PWN, Warszawa, $173 \mathrm{pp}$.

BERG H.-M. \& ZUNA-KRATKY Th. 1997. Rote Liste ausgewälter Tiergruppen Niederösterreichs - Heuschrecken und Fangschrecken (Insecta: Saltatoria, Mantodea). 1 Fassung 1995. NÖ Landesregierung, Abt. Naturschutz, Wien, $112 \mathrm{pp}$.

BERG M. \& KeLteR M. 2004. Die Gottesanbeterin, Mantis religiosa LINNAEUS, 1758 (Mantodea: Mantidae) im Stadtgebiet von Berlin-Schöneberg - Ihre Lebensweise und faunistische Beobachtungen in den Jahren 1998 bis 2003. Märkische Entomologische Nachrichten, Potsdam, 6: 55-84.

BRECHTEL F., EHRMANN R. \& DETzEL P. 1996. Zum Vorkommen der Gottesanbeterin Mantis religiosa L. (LINNE, 1758 ) in Deutschland. Carolinea 54:73-90.

BuCzyńska E., BuCzyńSKI P. \& PALKA K. 2006. Modliszka zwyczajna (Mantis religiosa L.) (Mantodea: Mantidae) na Roztoczu. Wiadomości entomologiczne, Poznań, 25: 56-57.

CHLADEK F. 1998. K současnému stavu rozšiřeníi kudlanky nábožné /Mantis religiosa Linnaeus, 1758/ na Moravě a poznámky k jeji biologii (Insecta, Mantoptera). Tetrix 1 (1): II 1998; www.volny.cz/tetrix.tetrix/

CMOLUCH Z. 1971. Nowe stanowisko Mantis religiosa L. (Mantodea) na Lubelszezyźnie. Przegląd Zoologiczny, Wrocław, 15: 374-375.

CZARNIAWSKT W., GOSTK R. \& WINTARCZYK S. 1999. Nowe dane o występowaniu modliszki zwyczajnej Mantis religiosa we wschodniej Małopolsee. Chrońmy Przyrodę Ojczystą, Kraków, 55 (5): 102-103.

Detzel P., Ehrmann R. 1998. Mantis religiosa (Linnaeus, 1758). In: Detzel P. (ed.), Die Heuschrecken BadenWürttembergs, pp. 181-187. Verlag Eugen Ulmer, Stuttgart, 580 pp.

DIETL [A.]. 1895. [Vereinsnachricht: Mantis religiosa]. Zeitschrift für Entomologie, Breslau, 20, p. XVIII.

DuRŁo G., WILCZYŃski S. 2006. Przykład zróżnicowania temperatury powietrza na polanie leśnej w Dolinie Kluczowody. Sylwan 7: 14-22.

EHRMANN R. 1984. Die Bedeutung der Cerci für Bildung der Oothek bei Mantis religiosa L. Articulata, Würzburg, 2 (4): $79-80$

EHRMANN R. 1985. Standorttreue von Mantis religiosa (L.). Articulata, Würzburg, 2 (7): 179-180.

EHRMANN R. 2003. Die Gottesanbetterin (Mantis religiosa), Neufunde in Deutschland. Articulata, Erlange, 18 (2): 253-254.

EISENBERG R. M. \& HURD L. E. 1993. Relative egg success and implications for distribution of three sympatric mantids (Mantodea: Mantidae). Proceedings of the Entomological Society of Washington, Washington, 95 : $271-277$.

Glowactíski Z., Bientek M., Dyduch A., Gertychowa R., Jakubiec Z., Kosior A. \& ZemaneK M. 1980. Stan fauny kręgowców i wybranych bezkręgowców Polski - wykaz gatunków, ich występowanie, zagrożenie i status ochronny. Studia Naturae, A, Warszawa-Kraków, 21, 163 pp.

GRZESIAK M., DOMAŃSKA W. (ed.) 2006. Ochrona środowiska 2006. Informacje i opracowania statystyczne. GUS, Warszawa, $521 \mathrm{pp}$.

GULIČKA J. 1992. Modlivka zelená (Mantis religiosa). In: Červená Kniha ohrozených a vzácnych druchov rastlin a živočichov ČSFR. Bezstavovce. Priroda, Bratislava, pp. 69-70.

HARZ K. 1957. Die Gottesanbeterin. Natur und Volk, Frankfurt am Main, 87: 187-193.

Karczewski J. 1955. Modliszka (Mantis religiosa L.) w Puszczy Sandomierskiej. Polskie Pismo Entomologiczne, Wrocław, 24, suppl. 1: 43 .

KATA K. 2004. Nowe informacje o występowaniu modliszki zwyczajnej Mantis religiosa L. na Podkarpaciu. Chrońmy Przyrodę Ojczysta, Kraków, 60 (4): 96-99.

KAWA P. 2001. Obserwacja modliszki zwyczajnej Mantis religiosa w powiecie Kolbuszowa. Chrońmy Przyrodę Ojczysta, Kraków, 57 (2): 116-117.

KOČAREK P. 2005. The order Mantodea. Řád Mantodea. In: Blattaria, Mantodea, Orthoptera and Dermaptera of the Czech and Slovak Republics, pp. 56-71. Kabourek, Zlin, 348 pp. 
KONDRACKI J. 1988. Geografia fizyczna Polski. PWN, Warszawa, 463 pp.

KRIŠTíN, A. \& MiHÁL, I., 2000: Rovnokrídlovee (Orthoptera) a modlivky (Mantodea) vybraných lokalít Národného parku Poloniny. Entomofauna carpathica 12: 37-40.

LIANA A. 1992. Owady prostoskrzydle Orthoptera. In: GLOWACחísKI Z. (ed.), Czerwona lista zwierząt ginących i zagrożonych w Polsce, pp. 85-91. Zakład Ochrony Przyrody i Zasobów Naturalnych, Kraków, 119 pp.

LIANA A. 1997. Świat zwierząt. In: LIANA A. (ed.), Na pograniczu regionów. Monografia przyrodnicza gminy Modliborzyce. pp. 55-122. Towarzystwo Fizjograficzne, Warszawa, 192 pp.

LIANA A. 2002a. Orthoptera prostoskrzydłe i inne owady ortopteroidalne. In: GŁoWACIŃSKI Z. (ed.), Czerwona lista zwierząt ginących i zagrożonych w Polsce, pp. 115-121. Instyt Ochrony Przyrody PAN, Kraków, 156 pp.

LiANA A. 2002b. Fauna prostoskrzydlych (Orthoptera) Lasów Janowskich. Nowy Pamiętnik Fizjograficzny, Warszawa, 1: 53-66.

LIANA A. 2004. Maniis religiosa (LNNAEUS, 1758). Modliszka zwyezajna. In: GlowaCIŃsKi Z., NowACKI J. (ed.), Polish Red Data Book of Animals. Invertebrates, pp. 72-73. IOP PAN, AR im. A. Cieszkowskiego, Kraków, 448 pp.

LORENC H. 2004. Klimat, wybrane zagadnienia. Ser. Instrukcje i podręczniki, pp. 9-36. Instytut Meteorologii i Gospodarki Wodnej, Warszawa, $104 \mathrm{pp}$.

LUKÁš, J. \& LUKÁŠovÁ Z. 2003. Distribution and parasitoids of prying mantid (Mantis religiosa, Linnaeus 1758) in Slovakia. Ochrana prírody 23: 242-252.

MICHALSKI J. 1959. Dwa dalsze stanowiska modliszki zwyczajnej (Mantis religiosa., Mantodea, Mantidae). Polskie Pismo entomologiczne, B, Wrocław, 3-4: 153-159.

PAWELEC J. \& BASISTA S. 2003. Inwazja modliszek w Magurskim Parku Narodowym. Parki Narodowe, Warszawa, 4/2003: okladka-1.

PAWLOWSKI J. 2003. Wstępna ocena dzialalności entomologicznej Karola PERTHÉESA. In: PAWLOWSKI J. (ed.), Karol Perthées (1739-1815) kartograf Pierwszej Rzeczypospolitej i entomolog, pp. 193-260. Rozprawy z dziejów nauki i techniki, 14. Komitet Historii Nauki i Techniki PAN, Warszawa, 342 pp.

PAX F. 1920. Beitrag zur Orthopterenfauna Schlesiens. Zeitschrift für wissenschaftliche Insektenbiologie, Husum, 16: $41-42$.

PISZKIEWICZ J., BENEŠ J. \& KONVIČKA M. 2000. Record of praying mantis, Mantis religiosa L., on the Velký Kosiř Hill, Central Moravia. Časopis Slezského Muzea v Opavě (A), Opava, 49: 58.

PRÜFFER J. 1955. Zmiany zachodzące w faunie owadów Polski obserwowane w latach ostatnich. Polskie Pimo entolologiczne, Wrocław, 24, suppl. 2: 85-111.

RAZOWSKI J. 1953. Nowe stanowisko modliszki w Polsce Mantis religiosa L. Wszechświat, Kraków, 2-3: 64.

RTCHLING A. \& OSTASZEWSKA K. 2006. Geografia fizyczna Polski. PWN, Warszawa, 345 pp.

SALT R. W. \& JAMES H. G. 1947. Low temperature as a factor in the mortality of eggs of Mantis religiosa L. Canadian Entomologist, Ottawa, 79: 33-36.

SCHOPPMANN A. 1989. Untersuchungen zur Populationsdichte einer Kolonie der Gottesanbeterin Mantis religiosa L. (Mantodea). Mitteilungen des Internationalen Entomologischen Vereins EV, Frankfurt/M, 14: 13-25.

SĘPIOL B. 2005. Nowe stanowisko modliszki zwyczajnej Mantis religiosa (Linnaeus 1758) na północy Krainy Gór Świętokrzyskich. Kulon, 10 (1-2): 77-79.

SKURATOWICZ W. 1946. Mało znane rezerwaty przyrodnicze Zamojszezyzny. Chrońmy Przyrodę Ojczystą, Kraków, 2 (3-4): 14-17.

ŠUŠLIK W. 1995. Faunistické a ekologické poznámky k modlivke zelenej (Mantis religiosa) na Slovensku. Entomomofauna Carpathica, $7(1-2): 2-3$.

THOMKA, V. 2003. Modlivka zelená - nové skutočnosti. Chránené územia Slovenska 55: 35.

VIDLIČKA L. 2001. Blattaria - šváby. Mantodea - modlivky (Insecta: Orthopteroidea). Fauna Slovenska. Veda, Bratislava, $171 \mathrm{pp}$.

VoISLN J. F. 2003. Atlas des Orthoptères et des Mantides de France. Muséum National d'Histoire Naturelle de Paris, $106 \mathrm{pp}$.

Waga A. 1843. O Janie Filipie Karozym (de Carosi) i Karolu Pertesie (de Perthées), dwóch naturalistach Polskich pod Stanislawem Augustem. Biblioteka Warszawska, 1843, 4: 193-210.

WiTKOWsKi Z. 1992. Mantis religiosa (LINNE, 1758). Modliszka zwyczajna. In: GLowaciński Z. (ed.), Polish Red Data Book of Animals, pp. 259-260. Państwowe Wydawnictwo Rolnicze i Leśne, Warszawa, 352 pp.

ZATOR A. 2004. Nalot ciepłolubnych. Dukielski Przegląd Samorządowy. Dukla, 3 (155) (http://www.dukla.pl/dps/dps0304/komentarze.htm)

ZIĘBA K. 2004. Nowe stanowisko modliszki zwyczajnej Mantis religiosa (Linné 1758) w poludniowo-wschodniej Polsce. Chrońmy Przyrodę Ojczysta, Kraków, 60, 4: 93-95. 


\section{STRESZCZENIE}

\section{[Zmiany w występowaniu Mantis religiosa (L.) w Polsce]}

Modliszka zwyczajna Mantis religiosa jest stałym elementem krajowej fauny, jednak w drugiej połowie XX wieku byla gatunkiem bardzo rzadko w Polsce spotykanym, o zasięgu ograniczonym do centralnej części Kotliny Sandomierskiej (Puszcza Sandomierska, Lasy Lipskie, Lasy Janowskie). Od 1984 roku została objęta ochroną ścisła, była też uznana za gatunek zagrożony wyginięciem. Do roku 1995 liczba znanych stanowisk krajowych nie przekraczała 20, wśród nich byly stanowiska watpliwe, lub pochodzące z krótkotrwałego zawleczenia. Na przelomie XX i XXI wieku wyraźny wzrost dynamiki zasięgu tego gatunku został odnotowany w niektórych krajach środkowej Europy (Niemcy, Czechy). W Polsce początkowo (1999 rok) zaobserwowano jedynie wzrost liczebności niektórych populacji w Puszczy Sandomierskiej, potem pojawianie się nowych stanowisk w obrębie stalego arealu, a także w innych regionach. W 2003 roku) modliszka zostala odnotowana po raz pierwszy w Beskidzie Niskim i na Pogórzu Środkowo-beskidzkim. Badania przeprowadzone w ramach grantu MNI w latach 2004-2006, w polączeniu z obserwacjami prowadzonymi w Kotlinie Sandomierskiej od 1995 roku, objęly ponad 550 stanowisk i pozwolily na stwierdzenie modliszki na 165 krajowych stanowiskach. Po uzupelnieniu danymi pochodzacymi od innych osób, lista obejmuje 208 stanowisk. Potencjalny krajowy zasię modliszki, wyznaczony przez jej skrajne stanowiska historyczne jest wprawdzie większy niż obecny, nigdy jednak zasięg nie byl tak zwarty i tak dobrze udokumentowany.

Aktualny krajowy zasięg $M$. religiosa jest zlożony z dwóch części: sandomierskiej, pulsujacej okresowo we wszystkich kierunkach (ze stalym refugium w centralnej części Kotliny Sanodmierskiej) oraz efemerycznej karpackiej, uzależnionej od dyspersji populacji slowackich. Cześć sandomierska obejmuje teraz także Wyżyny Malopolską (glównie Przedgórze Iłżeckie, Niecka Polaniecka i Pogórze Szydlowieckie) oraz Lubelską (Wzniesienia Urzędowskie i Kotlina Chodelska). Zmniejszające się zagęszczenie stanowisk ku pólnocy i zachodowi wskazuje na ich powiązanie i uzależnienie od centrum w Puszczy Sandomierskiej (ryc.3). Odizolowane stanowisko na Polesiu Lubelskim, odkryte w 2006 roku, pochodzi prawdopodobnie ze świeżego zawleczenia lub umyślnej introdukcji.

Badania wykazały poszerzenie spektrum ekologicznego gatunku. Klasycznymi środowiskami podgatunku $M$. religiosa polonica Baz. byly polany w borach sosnowych $\mathrm{z}$ mozaiką wrzosowisk oraz runa borowego, z domieszką roślinności psammofilnej. Obecnic ponad $60 \%$ stanowisk leży poza lasem i choć z reguly zachowują one charakter ekotonowy, mogą być zaliczone do środowisk otwartych raczej niż do śródleśnych. Glównym zasiedlanym przez pionierskie populacje środowiskiem sa kilkunastoletnie odłogi lub młodsze ugory na piaszczystych glebach, opanowywane przez murawy psammofilne Corynophoretum. Modliszka pojawia się coraz częściej w zupełnie nowych środowiskach, jak wilgotne ląki z Juncus effusus. Pojedyncze, migrujace samce obserwowane byly nawet na torfowiskach. Jednym z przejawów wzrostu dyspersji jest częstsze odnotowywanie przez przypadkowych obserwatorów przylatujących do światla samców modliszki.

Do glównych przyczyn wspólczesnej ekspansji $M$. religiosa trzeba zaliczyć fluktuacje klimatyczne w ostatnim dziesięcioleciu określane jako globalne ocieplenie. Modliszka jest gatunkiem wybitnie cieplolubnym, dlatego przy mniej sprzyjających warunkach termicznych jej występowanie na granicy zasięgu ogranicza się do najcieplejszych i najbardziej suchych regionów, w Polsce jest to centralna część Kotliny Sandomierskiej. Drugim czynnikiem jest obecność odpowiednich środowisk ekotonowych lub otwartych. W ostatnich kilkunastu latach w poludniowo-wschodnich regionach kraju nastapil znaczny przyrost powierzchni odłogów na glebach o niskiej bonitacji. Środowiska takie sa szybko opanowane przez roślinność 
psammofilną i kserofilną entomofaunę. Trzeci czynnik to aktywność ludzka ulatwiająca dyspersję gatunkom mało mobilnym. W przypadku modliszki przenoszone przez człowieka są prawdopodobnie glównie ooteki wraz z sianem, sadzonkami lub materialem budowlanym.

Najistotniejszym z zagrożeń dla modliszki może okazać się fluktuacja klimatu w kierunku odwrotnym niż obecny. Dane dotyczące historii klimatu w okresie ostatnich 250 lat, oraz informacje o występowaniu termofilnych owadów, świadczą, że podobne fluktuacje miały miejsce wcześniej i, że następowało po nich ochłodzenie, a w konsekwencji - recesja wielu gatunków. Zalesianie nieużytków przez człowieka bądź w drodze naturalnej sukcesji jest następnym zagrożeniem, tym większym, że towarzyszy mu coraz częstsze stosowanie herbicydów oraz insektycydów w uprawach leśnych, co może spowodować wymarcie trwałych, klasycznych populacji w Puszczy Sandomierskiej. Aby zmniejszyć zagrożenie modliszki oraz innych występujących w Puszczy gatunków bezkręgowców (m. in. Eresus niger, Scolia hirta, S. quadrimaculata, Calliptamus italicus, Podisma pedestris, Psophus stridulus) oraz kręgowców (np. Coronella austriaca) proponuje się wprowadzenie indywidualnych form ochrony (użytki ekologiczne, także śródleśne) oraz powołanie od dawna postulowanego Parku Krajobrazowego Puszczy Sandomierskiej. 